\title{
WestVirginiaUniversity
}

THE RESEARCH REPOSITORY @ WVU

Graduate Theses, Dissertations, and Problem Reports

2013

\section{Contact Lenses in Iris Recognition}

\author{
Gizem Erdogan \\ West Virginia University
}

Follow this and additional works at: https://researchrepository.wvu.edu/etd

\section{Recommended Citation}

Erdogan, Gizem, "Contact Lenses in Iris Recognition" (2013). Graduate Theses, Dissertations, and Problem Reports. 4965.

https://researchrepository.wvu.edu/etd/4965

This Thesis is protected by copyright and/or related rights. It has been brought to you by the The Research Repository @ WVU with permission from the rights-holder(s). You are free to use this Thesis in any way that is permitted by the copyright and related rights legislation that applies to your use. For other uses you must obtain permission from the rights-holder(s) directly, unless additional rights are indicated by a Creative Commons license in the record and/ or on the work itself. This Thesis has been accepted for inclusion in WVU Graduate Theses, Dissertations, and Problem Reports collection by an authorized administrator of The Research Repository @ WVU. For more information, please contact researchrepository@mail.wvu.edu. 


\title{
Contact Lenses in Iris Recognition
}

\author{
by \\ Gizem Erdogan \\ Thesis \\ submitted to the \\ College of Engineering and Mineral Resources \\ at West Virginia University \\ in partial fulfillment of the requirements \\ for the degree of \\ Master of Science \\ in \\ Computer Science \\ Bojan Cukic, Ph.D. \\ Katerina Goseva, Ph.D. \\ Arun Ross, Ph.D., Chair \\ Lane Department of Computer Science and Electrical Engineering \\ Morgantown, West Virginia \\ 2012
}

Keywords: Biometrics; Iris Recognition; Non-cosmetic Contact Lens; Dual Identity

Copyright 2012 Gizem Erdogan 


\author{
Abstract \\ Contact Lenses in Iris Recognition \\ by \\ Gizem Erdogan \\ Master of Science in Computer Science \\ West Virginia University \\ Arun Ross, Ph.D., Chair
}

Recent research in iris recognition has established the impact of non-cosmetic soft contact lenses on the recognition performance of iris matchers. Researchers in Notre Dame demonstrated an increase in False Reject Rate (FRR) when an iris without a contact lens was compared against the same iris with a transparent soft contact lens. Detecting the presence of a contact lens in ocular images can, therefore, be beneficial to iris recognition systems. This study proposes a method to automatically detect the presence of non-cosmetic soft contact lenses in ocular images of the eye acquired in the Near Infrared (NIR) spectrum. While cosmetic lenses are more easily discernible, the problem of detecting non-cosmetic lenses is substantially difficult and poses a significant challenge to iris researchers. In this work, the lens boundary is detected by traversing a small annular region in the vicinity of the outer boundary of the segmented iris and locating candidate points corresponding to the lens perimeter. Candidate points are identified by examining intensity profiles in the radial direction within the annular region. The proposed detection method is evaluated on two databases: ICE 2005 and MBGC Iris. In the ICE 2005 database, a correct lens detection rate of $72 \%$ is achieved with an overall classification accuracy of $76 \%$. In the MBGC Iris database, a correct lens detection rate of $70 \%$ is obtained with an overall classification accuracy of $66.8 \%$. To the best of our knowledge, this is one of the earliest work attempting to detect the presence of non-cosmetic soft contact lenses in NIR ocular images.

The second part of this thesis discusses the concept of dual identity, where a digital iris image hosts two distinct identities. In this regard, we design a method to alter a source iris image based on information from a target iris such that the modified iris retains the identity of both images. The proposed model strategically selects a minimal set of pixels from a target identity's iris pattern, copies it onto a source iris pattern, allowing the user to be able to be recognized both as his true identity and as the target identity. Experimental results based on 9506 pairs of source and target images suggest the efficacy of the scheme in rendering a digital iris capable of hosting two distinct identities. This model can potentially be employed to create patterned contact lenses facilitating dual identity by imprinting sparse patterns on transparent contact lenses. 


\section{Acknowledgements}

Firstly, I would like to thank my supervisor and my committee chair Dr. Arun Ross. I consider it an honor to be one of his students. I would not be able to succeed without his guidance, support and patience. His influence is not limited to my research but extends to my life.

I would like to thank Dr. Bojan Cukic and Dr. Katerina Goseva for being in my committee and for their invaluable input to my research.

I am thankful to all my lab mates in i-PRoBe Lab. They are the best friends and colleagues one could have.

I would like to acknowledge the contributions of all my professors in Lane Department of Computer Science and Electrical Engineering. I am also thankful to all the staff of West Virginia University for doing their jobs wonderfully.

I would like to thank all my friends in Morgantown for many beautiful memories we shared. Their presence gave me strength.

I would like to thank Ekrem Kocaguneli, who gave me the inspiration for graduate studies and always keeps on inspiring me.

Finally, I would like to thank my mother Yadigar, my father Ismail, my grandmother Husniye, and my grandfather Ali who raised me and supported me throughout my life with their unconditional love. 


\section{Contents}

Acknowledgements

List of Figures $\quad$ v

List of Tables $\quad$ viii

$\begin{array}{ll}\text { Notation } & \text { ix }\end{array}$

1 Introduction 1

2 Detection of Non-cosmetic Soft Contact Lenses $\quad 8$

2.1 Contact Lenses at a Glance . . . . . . . . . . . . . . . . . . . . . . . 8

2.1.1 Types of Contact Lenses . . . . . . . . . . . . . . . . . 8

2.1.2 Statistics on Contact Lenses _. . . . . . . . . . . . . . . . . 10

2.2 Related Work on Detecting Contact Lenses . . . . . . . . . . . . . . . . . . 14

2.3 Proposed Method . . . . . . . . . . . . . . . . . . . . . . . . . . . . . . . . . .

2.3.1 Traversal \#1 . . . . . . . . . . . . . . . . . . . . 18

2.3.2 Traversal \#2 . . . . . . . . . . . . . . . . . . . . . 19

2.4 Experimental Results . . . . . . . . . . . . . . . . . . 25

2.4.1 Image Level Experiments . . . . . . . . . . . . . . . . . 25

2.4.2 Subject Level Experiments . . . . . . . . . . . . . . . . . . . . . 29

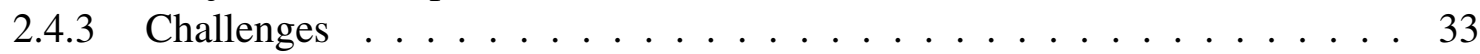

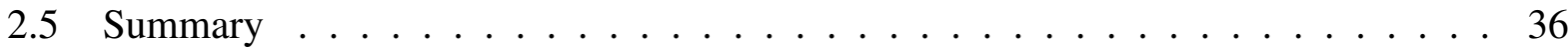

3 Generating a Dual Identity Iris Image $\quad 37$

3.1 Preliminary Examples . . . . . . . . . . . . . . . . . . . . . . . 39

3.2 Proposed Scheme . . . . . . . . . . . . . . . . . . . . 46

3.3 Experimental Results . . . . . . . . . . . . . . . . . . . 50

3.4 Feasibility of Spoof Attacks with the Proposed Model . . . . . . . . . . . . . . . 59

4 Conclusion $\quad \mathbf{6 0}$

4.1 Summary and Future Work . . . . . . . . . . . . . . . . . . 60

$\begin{array}{lc}\text { References } & 62\end{array}$ 


\section{List of Figures}

1.1 Anterior view of an iris in NIR spectrum. . . . . . . . . . . 2

1.2 Encoding phasor response as a bit pair. . . . . . . . . . . . . 3

1.3 An example demonstrating iris encoding. (a) An example eye image from ICE 2005 database. (b) Iris pattern normalized into pseudo polar coordinates. (c) Resultant iris template. (d) Corresponding iris mask. . . . . . . . . . . . . . . . 4

1.4 Same eye with cosmetic and non-cosmetic contact lenses, captured in the visible spectrum. (a) Eye with cosmetic lens. (b) Eye with non-cosmetic contact lens. (c) Eye without any contact lens. . . . . . . . . . . . . . . 5

1.5 Arrows indicating the lens perimeter that is visible against the sclera. . . . . . . . 7

2.1 Distribution of U.S. market share to different companies in 2011 [1] . . . . . . . 11

2.2 Distribution of contact lenses in the U.S. market according to material type (2011) [1]. 12

2.3 Distribution of soft and hard contact lens types in U.S. market in 2011 [1] . . . . . 12

2.4 Distribution of contact lens types due to replacement schedule in 2011 [1] . . . . . 13

2.5 Distribution of soft contact lens wear according to gender in 2000 [2]. . . . . . . 13

2.6 Distribution of soft contact lens wear according to different age groups in 2000 [2]. 14

2.7 The futulity of applying a canny edge operator to an NIR ocular image in order to detect contact lenses. The original image is given in (a). (b) Detected edges when threshold $=\{0.0024,0.06\}$ and $\sigma=\sqrt{2}$. (c) Detected edges when threshold $=\{0.0016,0.04\}$ and $\sigma=\sqrt{2}$. (d) Detected edges when threshold $=\{0.0008,0.02\}$

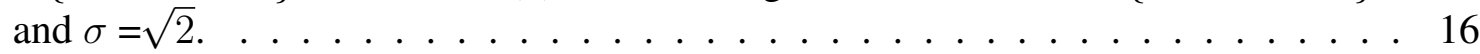

2.8 Preprocessing the input image. (a) Original eye image, whereas (b) Image after denoising. (c) Segmentation of outer iris boundary. . . . . . . . . . . . . .

2.9 Angular region considered for detecting the boundary of the lens. (a) Line segments extracted in the angular region $[\pi / 4$ and $3 \pi / 4]$. (b) Line segments extracted in the angular region $[-3 \pi / 4$ and $-\pi / 4]$. The union of the angular regions considered in (a) and (b) can be seen in (c). . . . . . . . . . . . . . . . . . .

2.10 Largest intensity pixels marked. (a) Largest intensity pixels on line segments in the angular region $[\pi / 4,3 \pi / 4]$. (b) Largest intensity pixels on line segments in the angular region [-3 $3 / 4$ and $-\pi / 4]$. (c) Union of the angular regions considered in (a)

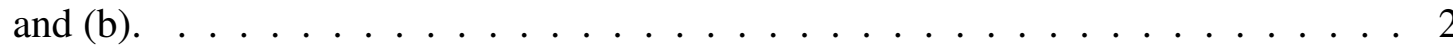


2.11 Clustering process. (a) Output the initial clustering process. Largest intensity pixels are illustrated in green and initial clusters are illustrated in yellow. (b) Reference cluster sets are illustrated in black. (c) The one remaining cluster is illustrated in red. . . . . . . . . . . . . . . . . . . . . . . . . 21

2.12 ROC curve for the image level experiment in the ICE 2005 dataset, based on varying $\alpha$ from 1 to 40 at $\tau=2 . \ldots \ldots \ldots \ldots \ldots \ldots$

2.13 ROC curve for the image level experiment in the ICE 2005 dataset, based on varying $\tau$ from 1 to 20 at $\alpha=7$.

2.14 ROC curve for the image level experiment in the MBGC Iris dataset, based on

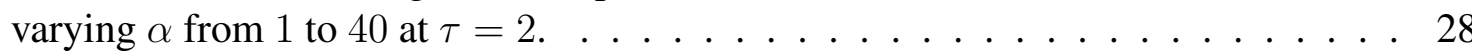

2.15 ROC curve for the image level experiment in the MBGC Iris dataset, based on varying $\tau$ from 1 to 20 at $\alpha=6 \ldots \ldots \ldots \ldots \ldots \ldots$

2.16 Effects of inconsistent illumination and camera defocus. (a) A case of inconsistent illumination, where the lens boundary is faintly visible in a with-lens image. (b) A case of inconsistent illumination, where a lens illusion is present in a without-lens image. (c) A case where the lens boundary is not distinguishable in a with-lens image due to defocus blur. . . . . . . . . . . . . . . . . .

2.17 ROC curve for the subject level experiment in the ICE 2005 dataset, based on varying $\alpha$ from 1 to 40 at $\tau=4$.

2.18 ROC curve for the subject level experiment in the ICE 2005 dataset, based on varying $\tau$ from 1 to 20 at $\alpha=9$.

2.19 ROC curve for the subject level experiment in the MBGC Iris dataset, based on varying $\alpha$ from 1 to 40 at $\tau=4$.

2.20 ROC curve for the subject level experiment in the MBGC Iris dataset, based on varying $\tau$ from 1 to 20 at $\alpha=9$.

2.21 Examples of unsuccessful classification cases. (a) An example of an insignificant lens boundary. (b) An example of eyelid interference. (c) Inaccurate segmentation of the limbus boundary, which leads to misclassifying a with-lens image. (d) Inaccurate segmentation of the limbus boundary, which causes misclassification of a without-lens image. (e) An example where the lens is misplaced in the eye. . . . .

3.1 Overview of the proposed model. (a) Creation of the composite iris pattern. (b) Production of the patterned contact lens. . . . . . . . . . . . . . . 38

3.2 Example of a source identity's iris information. (a) Eye image of the source identity. (b) Source identity's normalized iris pattern. (c) Source identity's iris template. (d) Corresponding mask for the source identity's iris template. . . . . . . . . 40

3.3 Example of a target identity's iris information. (a) Eye image of the target identity. (b) Target identity's normalized iris pattern. (c) Target identity's iris template. (d) Corresponding mask for the target identity's iris template. . . . . . . . . . . .

3.4 A trivial solution: Stitching halves of normalized images from the source and the target. (a) Iris pattern obtained by stitching. (b) Iris template of the stitched image.

(c) Corresponding mask for iris template of the stitched image. . . . . . . . . . 
3.5 A trivial solution: Mixing normalized iris images uniformly. (a) Iris pattern obtained by uniformly mixing two normalized iris patterns. (b) Iris template of the uniformly mixed image. (c) Corresponding mask for iris template of the uniformly mixed image. . . . . . . . . . . . . . . . . . . .

3.6 An alternative approach: Mixing normalized iris images randomly. (a) Iris pattern obtained by randomly mixing two normalized iris patterns. (b) Iris template of the randomly mixed image. (c) Corresponding mask for iris template of the randomly mixed image.

3.7 A demonstration of the proposed scheme. (a) Source iris pattern $P_{1}$. (b) Target iris pattern $P_{2}$. (c) Pixels to be copied. (d) The resultant composite iris pattern. . . . . . 48

3.8 Example \#1: Source, target and composite iris patterns with the corresponding HDs. 50

3.9 Example \#2: Source, target and composite iris patterns with the corresponding HDs. 51

3.10 Example \#3: Source, target and composite iris patterns with the corresponding HDs. 51

3.11 Example \#4: Source, target and composite iris patterns with the corresponding HDs. 51

3.12 Example \#5: Source, target and composite iris patterns with the corresponding HDs. 52

3.13 Example \#6: Source, target and composite iris patterns with the corresponding HDs. 52

3.14 Example \#7: Source, target and composite iris patterns with the corresponding HDs. 52

3.15 Example \#8: Source, target and composite iris patterns with the corresponding HDs. 53

3.16 Example \#9: Source, target and composite iris patterns with the corresponding HDs. 53

3.17 Example \#10: Source, target and composite iris patterns with the corresponding HDs. . . . . . . . . . . . . . . . . . . . 53

3.18 Distribution of HD between composite iris and the source and target iris patterns. . 54

3.19 Histogram of the percentage of pixels copied onto the source image. . . . . . . . . 54

3.20 Distribution of HD between different patterns. Distribution of HD between genuine patterns are shown with magenta. . . . . . . . . . . . . . . 55

3.21 Distribution of HD between different patterns. Distribution of HD between the composite pattern and the other samples of its source identity are shown in cyan. Distribution of HD between the composite pattern and the other samples of its target identity are shown in black. . . . . . . . . . . . . . 56 56

3.22 Example \#11: The pair of source and target images which have the highest HD. . . 56

3.23 Example \#12: The case where the HD between the source image and the composite image is the highest. . . . . . . . . . . . . . . . . 57

3.24 Example \#13: The case where the HD between the target image and the composite image is the highest. . . . . . . . . . . . . . . . 57

3.25 Example \#14: The case where the sum of the HDs between the composite and the target image and the HD between the composite and the source image is the highest. 57

3.26 Example \#15: The case with the highest percentage of pixels copied, and the related results. . . . . . . . . . . . . . . . . . . 58 


\section{List of Tables}

2.1 Confusion matrix for the image level experiment in the ICE 2005 dataset, where

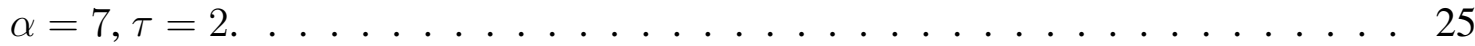

2.2 Confusion matrix for the image level experiment in the MBGC Iris dataset, where

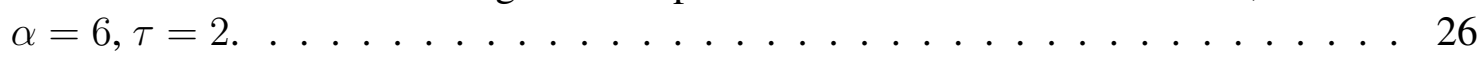

2.3 Confusion matrix for the subject level experiment in the ICE 2005 dataset where

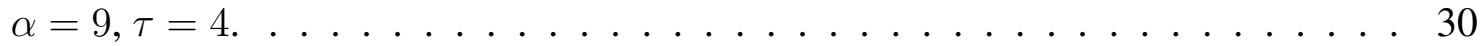

2.4 Confusion matrix for the subject level experiment in the MBGC Iris dataset where

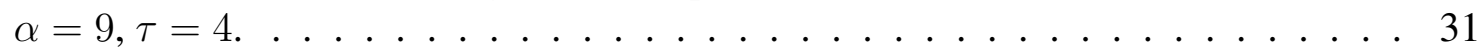




\section{Notation}

The notation and the symbols used throughout this document are as follows:

$D P I \quad$ : Dots per Inch

FPR : False Positive Rate

$F R R$ : False Reject Rate

$H D$ : Hamming Distance

NIR : Near Infrared

$R G P$ : Rigid Gas Permeable

$T P R$ : True Positive Rate 


\section{Chapter 1}

\section{Introduction}

The iris is an internal organ of the eye which is located between the cornea and the lens $[3,4]$. The most important function of the iris is controlling the amount of light that reaches the retina through the pupil. The iris regulates the size of the pupil by dilating and contracting the pupil with the dilator and sphincter muscles. There are two boundaries contouring the iris: the pupillary boundary which lies between the pupil and the iris, and the limbus boundary which lies between the iris and the sclera. The iris consists of two layers: the visible, lightly pigmented one is the anterior layer and the less visible, darkly pigmented one is the posterior layer. Muscles and vascular tissue are found in between these layers. The anterior layer consists of the pupillary area and the ciliary area and their boundary is called collarette. Both areas contain a variety of structures such as radial and concentric furrows, pigment piles and periphery crypts. Figure 1.1 shows the anterior view of an iris captured in NIR spectrum. All iris structures, along with the muscles and vessels, form an intricate textural iris pattern. Due to the random morphogenesis of the iris pattern, even two irides with the same genetic genotype (as in identical twins, or the pair possessed by one individual) have been observed to be unique [5].

The process of establishing human identity based on iris patterns is known as iris recognition. Iris recognition is performed by comparing a subject's iris pattern to number of gallery images acquired (in NIR spectrum) from the users enrolled in the system. The process starts by localizing the pupillary and limbus boundaries of the eye and segmenting the iris region. Further, the iris area is isolated from any eyelid/ eyelash occlusions or specular reflections. The annular-like segmented iris is then geometrically normalized into a rectangular region using a rubber-sheet model 


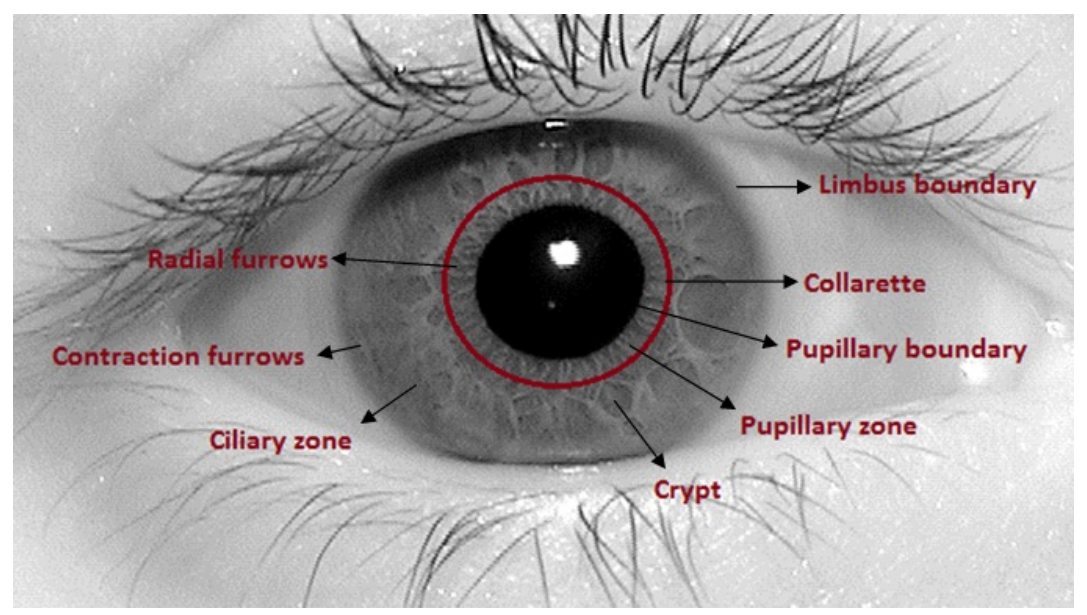

Figure 1.1: Anterior view of an iris in NIR spectrum.

in pseudo polar coordinates. Creating the iris template from the normalized image is called encoding. Iris encoding is performed by convolving the normalized iris with Gabor wavelets. The result is a set of complex numbers consisting of one number for every pixel in the normalized image. The amplitudes of the complex numbers are discarded, since amplitude is shown to be dependent on environmental factors such as illumination and camera gain. The phase information of each complex number is encoded into two bits per pixel, to form an iris template that is double the length of the polar iris image. Figure 1.2 demonstrates how quadrant location of a complex response $z$ is converted to a pair of bits. Figure 1.3 shows an example eye, its normalized iris pattern and the resultant iris template.

Iris matching is carried by measuring the hamming distance (HD) between different iris templates. If the HD between two images is below a certain threshold, these images are considered to be from the same eye. Daugman suggests that a threshold of HD $\leq 0.32$ will distinguish iris patterns with high confidence [6].

Contact lenses are placed in the eye to correct vision or for aesthetic purposes [7]. There are several taxonomies for categorizing contact lenses. The most commonly used taxonomy differentiates contact lenses based on the material used to manufacture them [8]. Contact lenses are classified into two main groups in this respect: soft contact lenses and rigid gas permeable (RGP) contact lenses. Soft contact lenses are made of soft, flexible, highly absorbent plastics called hydrogels. Hydrogels are hydrophilic materials, which are hard in the dry state but become soft 


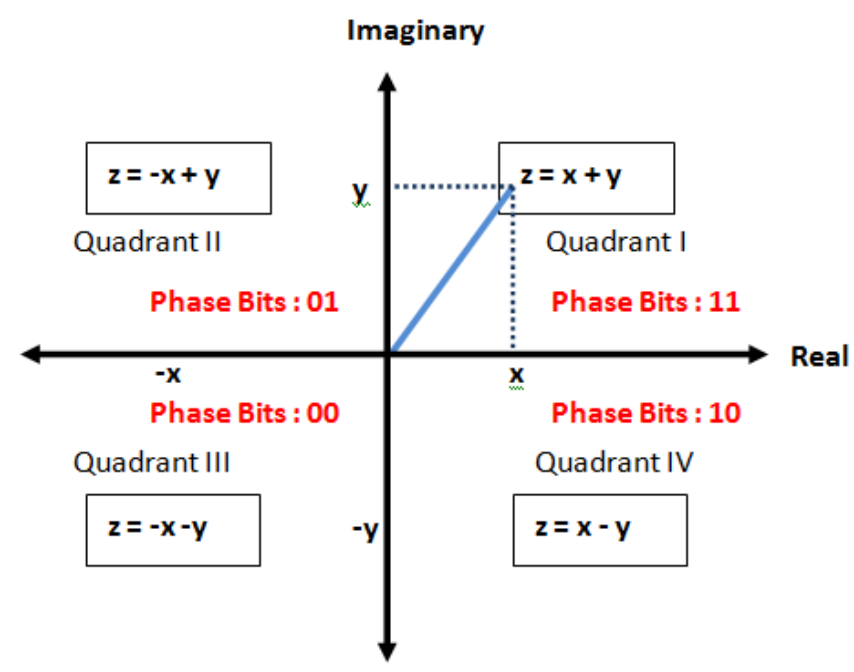

Figure 1.2: Encoding phasor response as a bit pair.

when they absorb water [9]. When they are soft, they allow oxygen to pass through. They fit comfortably in the eye. An advanced type of soft contact lenses, called Silicone Hydrogel, was released in 1998. This type of lens provides more oxygen flow than regular hydrogels, and reduces eye irritations. [8]. RGP contact lenses constitute the second main group and are generally used as a solution for complicated spectacle prescriptions, since they result in a clearer vision than soft contact lenses. RGP contact lenses are made of more durable plastic than soft contact lenses. However this makes them less comfortable to the wearer and harder to adapt to [8]. Recently, hybrid lenses, made by mixing soft and RGP lenses, have been produced to facilitate the design of a more customized lens type. Hybrid lenses are rigid in the center of the lens but softer in the edges [7]. However, the usage of this type of lens is limited to a small number of users for now [1].

Soft contact lenses can be divided into two types based on their function:

1. Cosmetic soft contacts, which are worn to visually alter the appearance of the eye;

2. Non-cosmetic soft contacts, which are prescribed by optometrists for correcting vision for therapeutic purposes.

Soft contact lenses can be divided into two types based on their function:

1. Cosmetic soft contacts, which are worn to visually alter the appearance of the eye; 


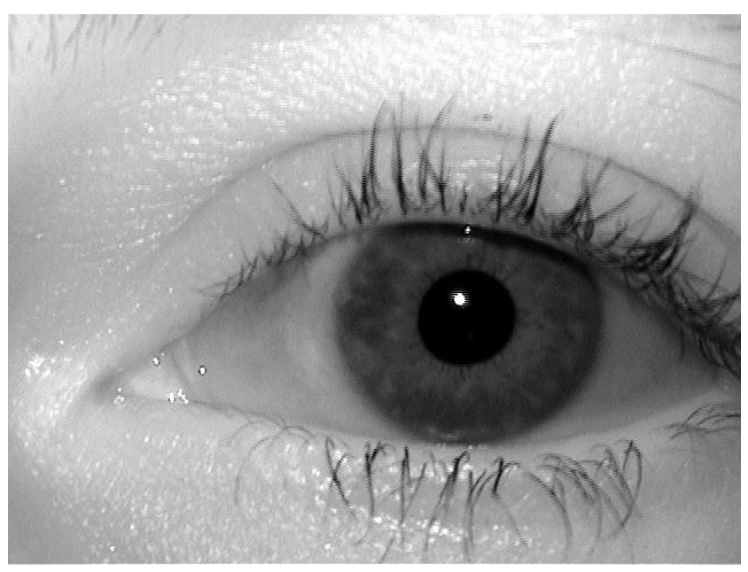

(a)

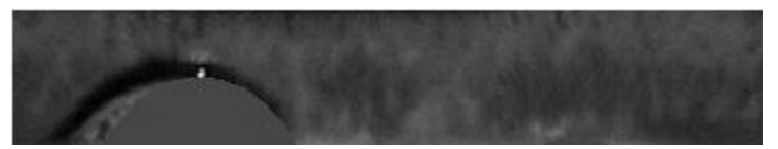

(b)

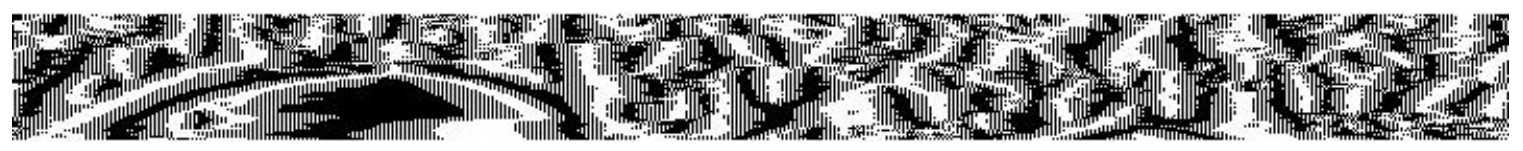

(c)

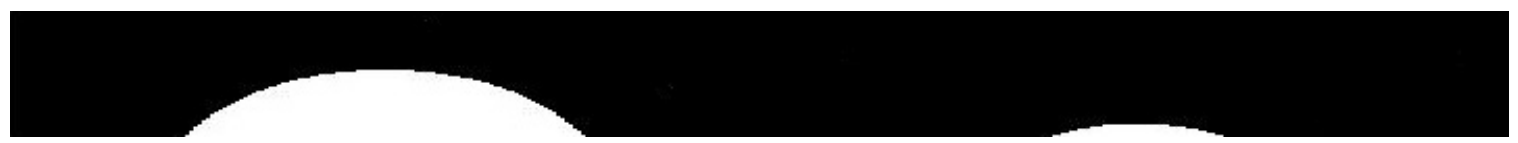

(d)

Figure 1.3: An example demonstrating iris encoding. (a) An example eye image from ICE 2005 database. (b) Iris pattern normalized into pseudo polar coordinates. (c) Resultant iris template. (d) Corresponding iris mask.

2. Non-cosmetic soft contacts, which are prescribed by optometrists for correcting vision or for therapeutic purposes.

Cosmetic contact lenses are colored and opaque, and can hide the actual color of the eye. They come in a variety of colors and designs, yet they only account for approximately 3 to $5 \%$ of all 
the soft contact lenses sold in the US market [2]. While cosmetic lenses can also correct refractive errors in the eye, this is considered to be a secondary purpose of use. An example image of an eye with a cosmetic contact lens can be seen in Figure 1.4(a). Non-cosmetic lenses, on the other hand, are colorless lenses. However, some of them have a tint that makes it easy to locate the lens if it is dropped or misplaced. Likewise, they may have letters like " $\mathrm{L}$ " or " $\mathrm{R}$ " to facilitate their correct handling and placement. An example image of an eye with a non-cosmetic contact lens can be seen in Figure 1.4(b). The same eye without any contact lenses can be seen in Figure 1.4(c).

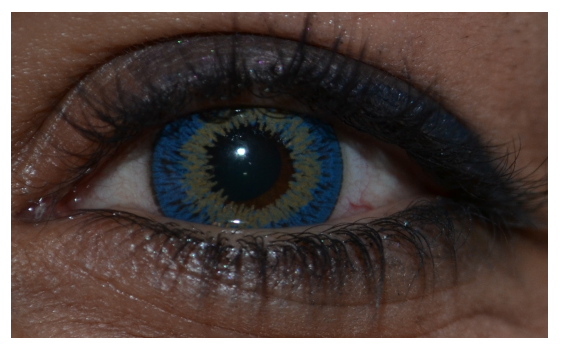

(a)

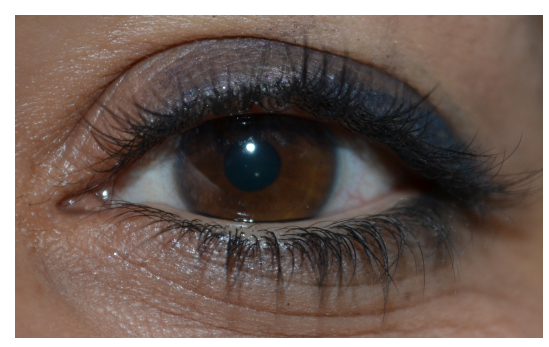

(b)

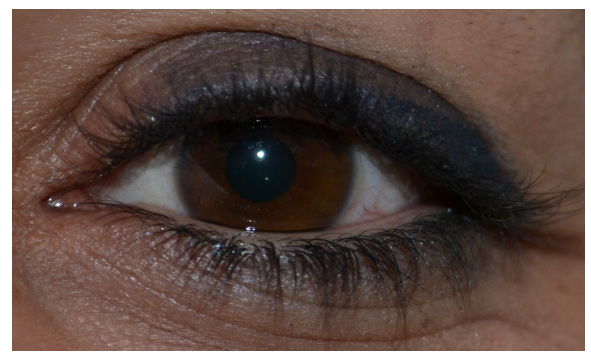

(c)

Figure 1.4: Same eye with cosmetic and non-cosmetic contact lenses, captured in the visible spectrum. (a) Eye with cosmetic lens. (b) Eye with non-cosmetic contact lens. (c) Eye without any contact lens.

Since cosmetic lenses completely conceal the iris by obscuring it with a fake iris pattern, the detection of such lenses has been studied extensively in the context of iris spoofing [10-14]. On the other hand, non-cosmetic lenses are not intended to obscure or alter the characteristics of the iris. This may lead one to assume that non-cosmetic lenses have negligible effect on iris recognition performance. However, such an assumption was demonstrated to be incorrect by Baker et al. [15]. 
Their experiments showed a degradation in iris recognition performance proportional to the size of the artifact the contact lens leaves on the iris. They also showed that, the presence of non-cosmetic contact lenses, which are almost imperceptible once placed in the eye, still decrease the recognition performance.

Baker et al. categorized contact lenses into four categories according to the artifact present on the iris due to a contact lens. The first category consisted of non-cosmetic soft contact lenses which do not have any visible artifacts on the iris. The second category consisted of non-cosmetic soft contact lenses which create a thin circular outline on the iris. The third category consisted of lenses with larger artifacts such as letters and numbers, and ill-fitting lenses. Lastly, in the fourth category, they placed iris images with RGP contacts. The results of their experiments using three different recognition systems showed that matching images without contact lenses outperform all other categories. On average:

- the matches between images in the fourth category of lenses experienced a False Reject Rate (FRR) about 35 times that of the matches between images without any contact lenses.

- the matches between images in the third category of lenses experienced a FRR about 4 times that of the matches between images without any contact lenses.

- the matches between images in the second category of lenses experienced a FRR about 2 times that of the matches between images without any contact lenses.

- the matches between images in the first category of lenses experienced a FRR about 1.5 times that of the matches between images without any contact lenses.

The results of the study by Baker et al. suggest that iris matchers can benefit from the information about the presence or absence of non-cosmetic contact lenses in iris images. In particular, watch-list scenarios, where high false reject rates create security threats, can benefit highly from such information. This is especially necessary due to the widespread use of non-cosmetic lenses in the population and the number of biometric applications that are beginning to embrace iris recognition.

In the first part of this thesis, a method for non-cosmetic soft contact lens detection is proposed. The focus of the proposed method is on soft contact lenses, which usually extend beyond the 
cornea, where a lens border is visible in the sclera, yet without any perceptible artifacts on the iris. A sample case is shown in Figure 1.5.

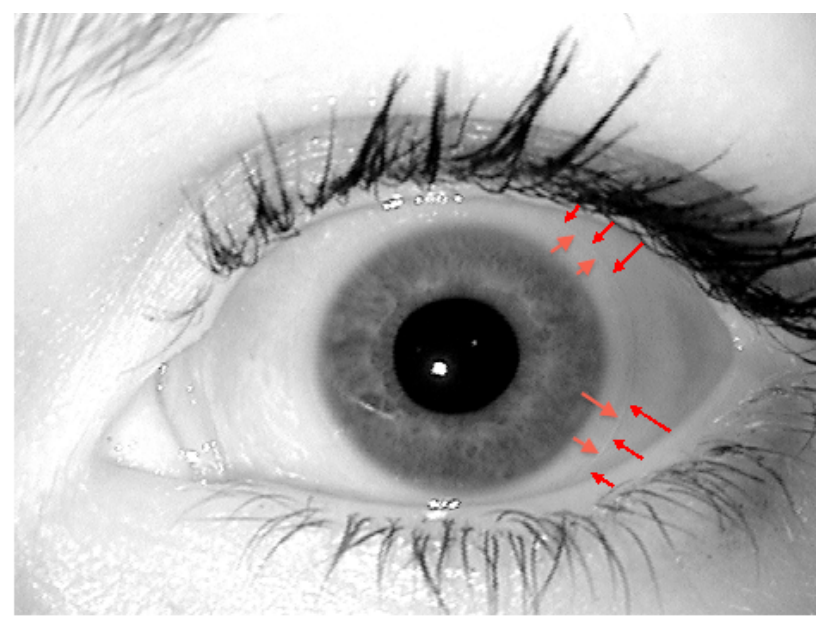

Figure 1.5: Arrows indicating the lens perimeter that is visible against the sclera.

The proposed method detects the lens boundary through a two phase traversal of a small annular region located in the vicinity of the segmented limbus boundary. In each phase of the traversal, it considers groups of pixels and examines the intensity profiles in these groups. Profiles that satisfy a set of constraints are considered as candidate points, suggesting the existence of a lens perimeter.

In the second part of this thesis, we propose a model for creating a dual-identity iris image. The proposed model copies certain pixels from a target identity's iris pattern onto a source iris pattern. This model can potentially be used to create patterned contact lenses that would allow the user to be able to be recognized both as his true identity as well as the target identity.

The rest of this thesis is organized as follows: Chapter 2 provides a description of contact lenses available in today's market, discusses previous work in the literature on detecting cosmetic and non-cosmetic lenses, provides details of the proposed non-cosmetic contact lens detection method, reports experimental results and discusses the challenges encountered. Chapter 3 introduces a method for creating a dual identity digital iris and evaluates the proposed method. Chapter 4 summarizes the main contributions and concludes the paper. 


\section{Chapter 2}

\section{Detection of Non-cosmetic Soft Contact}

\section{Lenses}

\subsection{Contact Lenses at a Glance}

\subsubsection{Types of Contact Lenses}

Contact lenses can be be categorized in many different ways [2]:

- By material type: This is the most common classification used and was already explained in Chapter 1.

- Soft contacts

- RGP contacts

- By replacement frequency:

- Disposables:

* Daily disposables: This type of lenses are worn for a day and then discarded. The advantage of such lenses is that, a fresh lens is used daily, and, hence the user does not have to purchase any cleaning or care products. These lenses are especially suitable for sensitive eyes and allergic users ${ }^{1}$. However, they are usually costly to use on a full-time basis.

\footnotetext{
${ }^{1}$ Contact lens allergies can occur when contact lenses themselves or the proteins that bind to the surface of the lens cause an irritation in the eye surface. The symptoms of contact lens allergies include redness, itching, mucous
} 
* Weekly disposables: This type of lenses are generally cheaper than daily disposables. However they should be maintained clean and disinfected before reinserting. The wearers usually find the weekly replacement schedule easy to comply with because it is easy to remember [17].

- Planned replacements: This type of lenses require cleaning and disinfecting after every period of wear. One should note that the schedules are defined by the time between first use and the final disposal of the lens, regardless of the actual amount of usage in between. Planned replacement contacts usually have more choices than disposables, and thus better fit may be obtained with this kind of lenses.

* Bi-weekly: This type of lenses is replaced every two weeks.

* Monthly: This type of lenses requires changing every month.

* Quarterly: This type of lenses requires changing every three months.

- Conventional contacts:

* 1 year: This type of lenses can be used up to one year but they are not commonly prescribed any more.

- By sleeping usage:

- Daily wear: This type of lenses is worn during waking hours only.

- Flexible wear: This type of lenses is for daily wear. However irregular, occasional sleeping with the lenses is also possible.

- Extended wear: This type of lenses is for regular sleeping for a maximum of six nights, followed by a night without lenses.

- Continuous wear: This type of lenses is for regular sleeping for up to 30 days or 30 nights.

- By correction modality:

discharge, and lens discomfort. A more severe type of contact lens allergy may result in not being able to wear lenses due to large swellings in the mucous membrane of the upper eyelid [16]. 
- Spherical lenses: This type of lenses is the regular contact lenses which refract light evenly in every direction. They are used to correct myopia and hyperopia and they come in both soft or RGP forms.

- Toric (Astigmatic) lenses: Toric contact lenses are specially designed for people with astigmatism [18]. These lenses are made from the same material as other contact lenses and come in soft or rigid gas permeable forms. Toric lenses have two different curvatures allowing correction for both astigmatism and myopia or hyperopia. Because they must maintain the same orientation, toric lenses must be inserted properly, thus they often are marked with indicators on the sides or bottom to aid in insertion. These lenses are often designed to be heavier at the bottom so that the lens will automatically rotate into correct position after blinking or other movement.

- Bifocal lenses: Bifocal contact lenses are designed for people who have presbyopia, the age-related, decreased ability to see both near and far distances [18]. A bifocal contact lens design has both the distance prescription and near prescription in one lens. They are similar to multi-focal eyeglasses in these terms, that is they use different optical zones to correct presbyopia. They come in both soft and RGP forms.

\subsubsection{Statistics on Contact Lenses}

The Contact Lenses 2011 report, which is published by Contact Lens Spectrum [1], provides data obtained from Robert W. Baird \& Co., about US contact lens market. According to this study, worldwide contact lens market in 2011 is estimated at $\$ 6.8$ billion, while the U.S. contact lens market is estimated at $\$ 2.6$ billion.

Four major lens manufacturers share the contact lens market in USA [7] :

- Bausch \& Lomb (B\& L): makers of various soft lens brands.

- Ciba Vision: makers of Ciba brand lenses.

- CooperVision: makers of Biofinity and Proclear brand lenses.

- Johnson \& Johnson (J\& J): makers of Acuvue brand lenses. 
The leading company in U.S. contact lens market is J\& J with $42 \%$ share. J\& J is followed by Ciba Vision at 25\%, CooperVision at $21 \%$, and B\& L at $12 \%$. The shares generating the chart of Figure 2.1 belong to 2011 fiscal year [1].

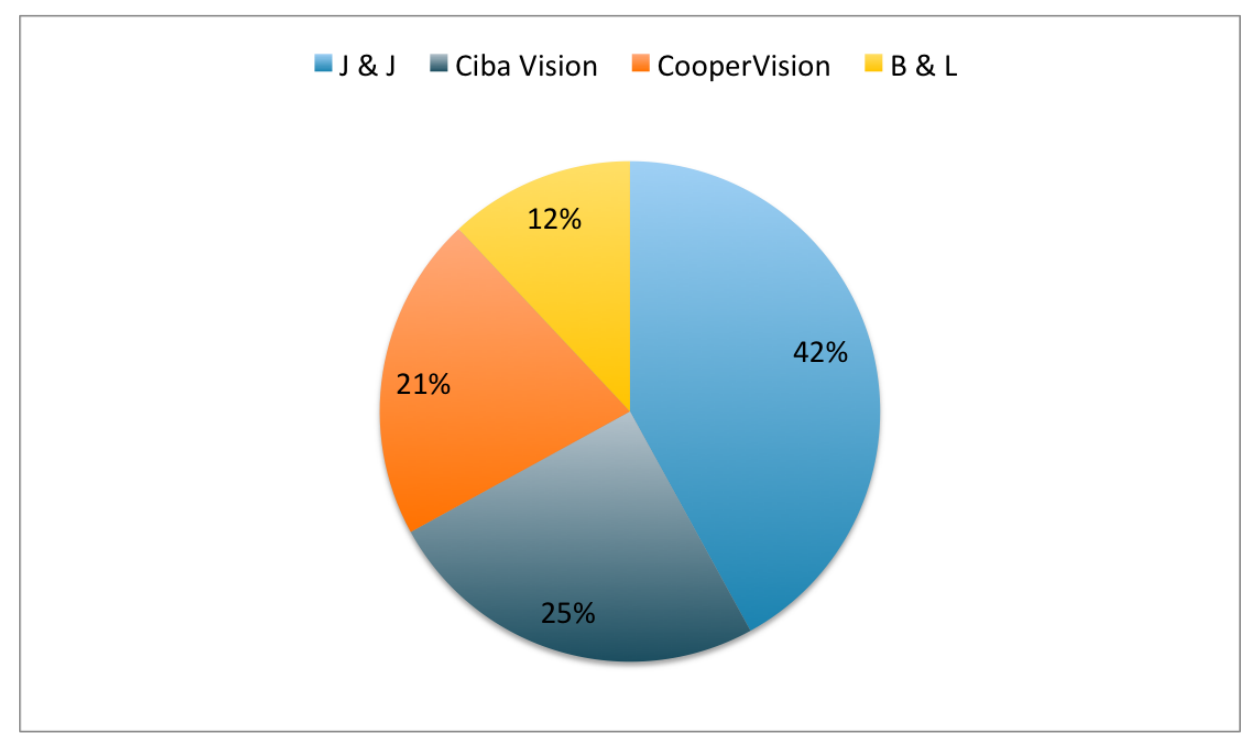

Figure 2.1: Distribution of U.S. market share to different companies in 2011 [1].

Figure 2.2 visualizes the distribution of different classes of materials used to manufacture contact lenses that were sold in the U.S. market in 2011 [1]. It can be seen that soft contact lenses dominate the market with $91 \%$.

Figure 2.3 illustrates the distribution of soft and hard contact lens types in the market due to correction modality [1]. As shown in the figure, the largest market share belongs to "spherical soft" lenses with a percentage value of 54\%. It is followed by "toric soft lenses", whose market share is $23 \%$. The third biggest share in the market belongs to "multifocal soft lenses" with a value of $11 \%$. The rest of the market is shared by various different types of hard contact lenses.

Many types of lenses are manufactured according to different usage schedules. Figure 2.4 gives information regarding the replacement schedules of the lenses in the market in 2011 [1]. According to Figure 2.4, the most preferred type in the market is monthly scheduled lenses, with a percentage share of $46 \%$. This is followed by biweekly lenses with a share of $33 \%$. The third biggest share belongs to daily contacts, with $15 \%$. Daily contacts are followed by quarterly, yearly and weekly scheduled contact lenses, respectively. 


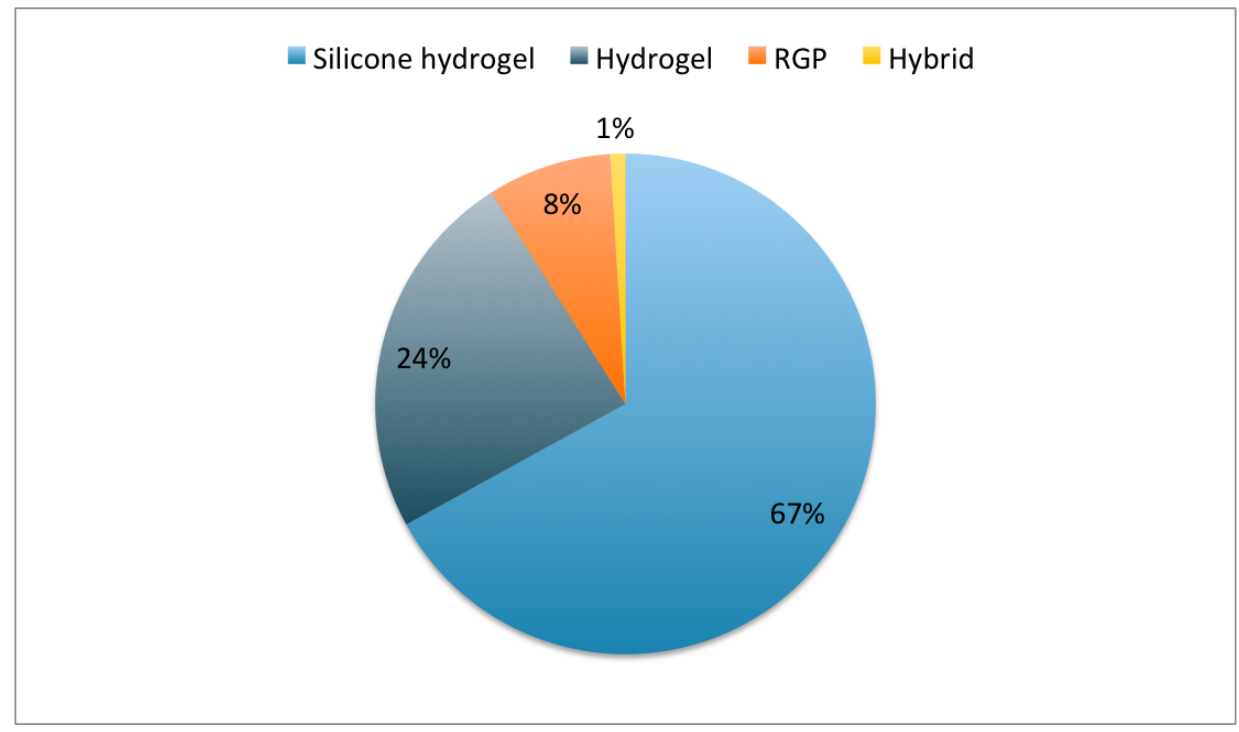

Figure 2.2: Distribution of contact lenses in the U.S. market according to material type (2011) [1].

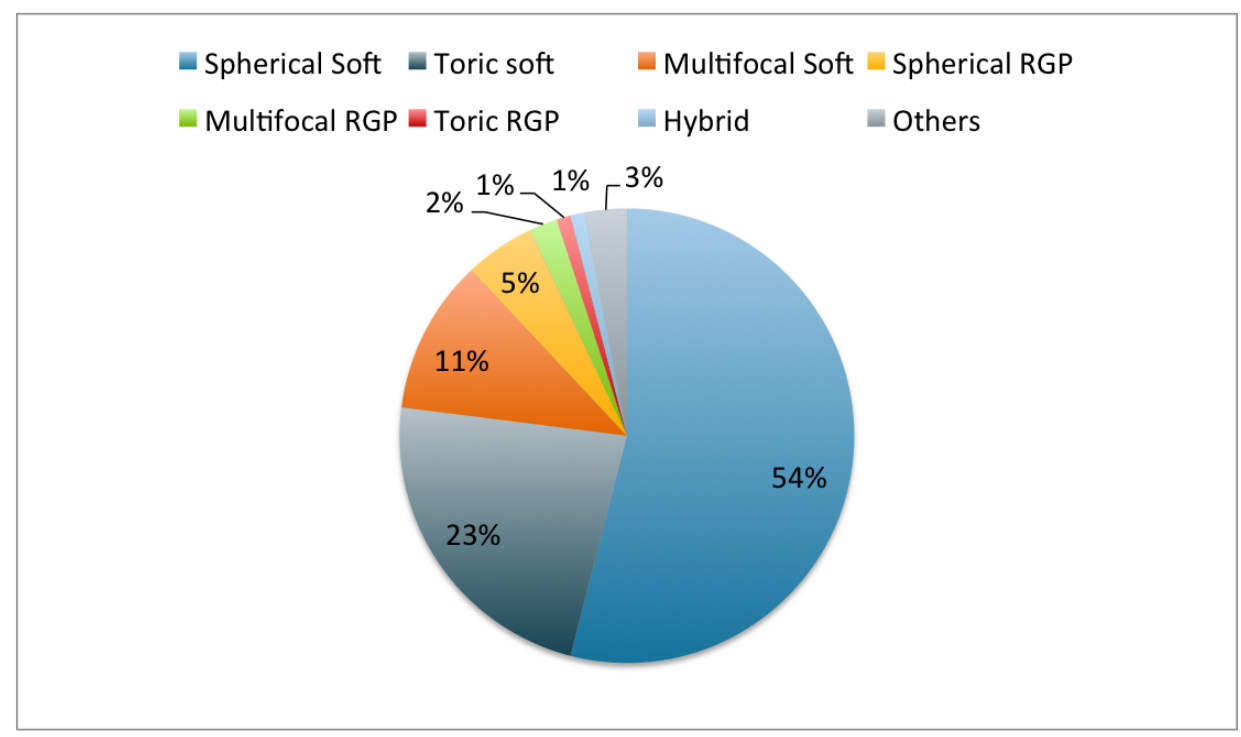

Figure 2.3: Distribution of soft and hard contact lens types in U.S. market in 2011 [1].

Figure 2.5 shows how lens usage distributes across genders in U.S. market in 2000. Females are the dominant customers of contact lenses. $72 \%$ of all the contact lenses sold in the year of 2000 were bought by female customers. The rest (28\%) were bought by male customers [2].

Figure 2.6 provides the distribution of the age groups of the contact lens customers in 2000 . The biggest segment is composed of customers between the ages of 26 and 39. Of all the contact 


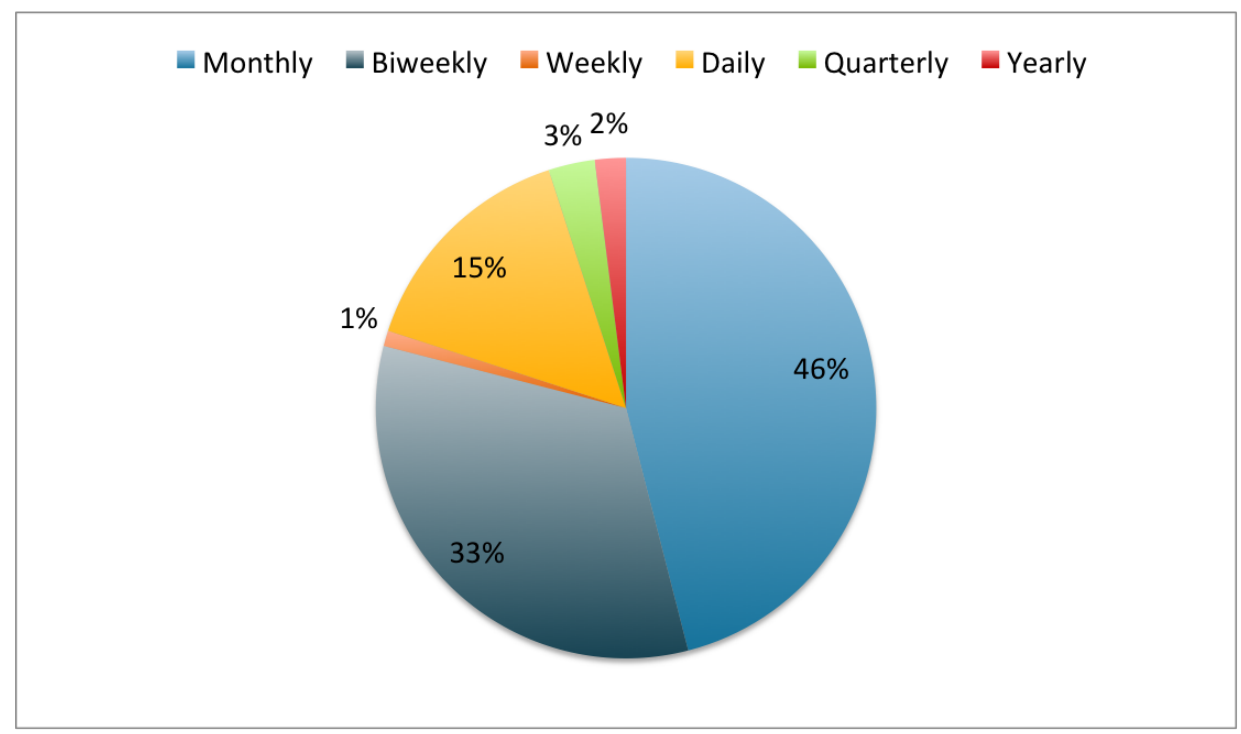

Figure 2.4: Distribution of contact lens types due to replacement schedule in 2011 [1].

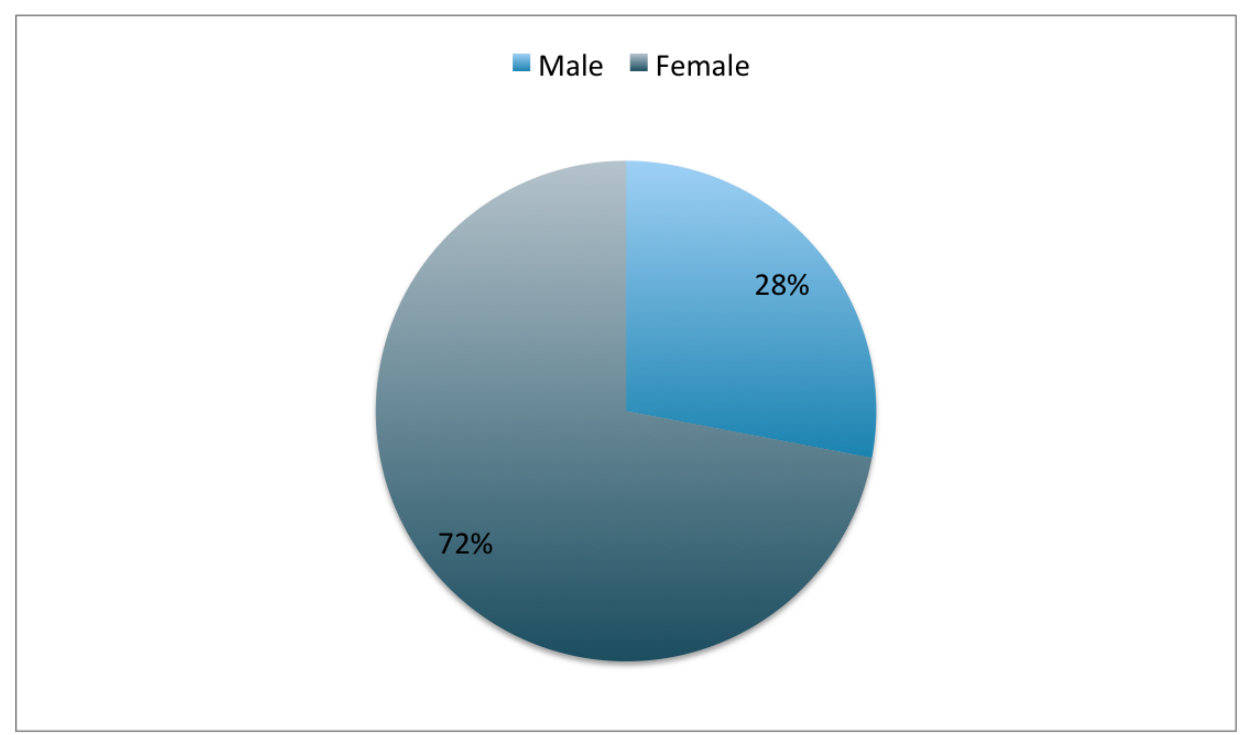

Figure 2.5: Distribution of soft contact lens wear according to gender in 2000 [2].

lenses sold, this segment bought $45 \%$. The second biggest age segment is "18 to 25 " followed by the age group of " 40 and older"; these age groups have the market shares of $23 \%$ and $22 \%$, respectively. The smallest market share belongs to the age group of "17 and under" [2].

One of the implications of this statistics is that, contact lenses drive an important commercial market. They are used by both male and female users from all age groups. Furthermore, contact 


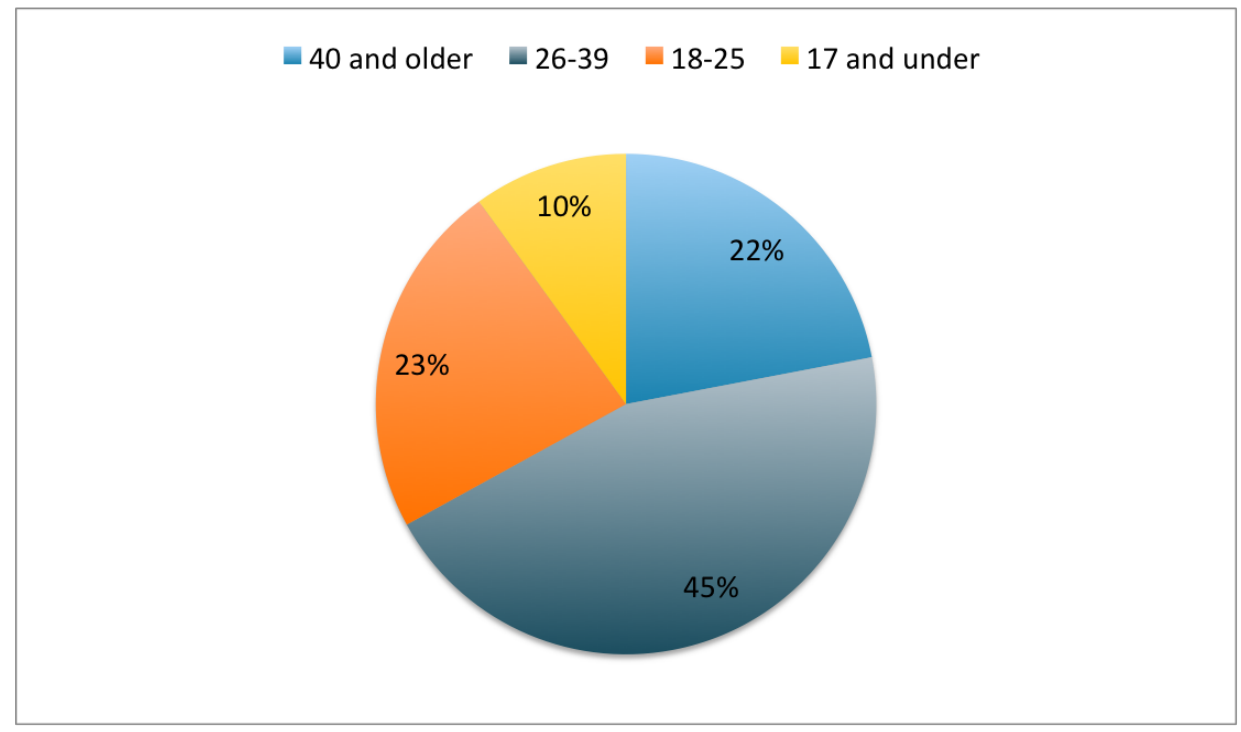

Figure 2.6: Distribution of soft contact lens wear according to different age groups in 2000 [2].

lenses have a wide range of functional variety and many different sub-types according to a number of criteria, such as correction modality, replacement frequency and sleeping usage. However, more than $90 \%$ of all the contact lenses in the market are non-cosmetic soft contacts, which our study focuses on.

\subsection{Related Work on Detecting Contact Lenses}

There has been significant research effort invested in the detection of cosmetic contact lenses, since cosmetic lenses are associated with the potential problem of iris spoofing and obfuscation. Daugman [14] proposed the use of Fast Fourier Transform (FFT) to detect cosmetic contact lenses by exploiting the periodicity of the dot-matrix printing process. Lee et al. [12] presented a technique to distinguish the Purkinje images (reflected images of incident light on the four optical surfaces of the eye) of a live iris from that of a cosmetic lens. They theoretically established the locations and distances between the Purkinje images on a real human eye and showed that the corresponding distances on a fake eye (including digital spoofs, prosthetic eye, cosmetic lens) are significantly different. He et al. [10] proposed to detect cosmetic contact lenses using statistical texture analysis and support vector machines. Wei et al. [13] introduced 3 different methods. Their 
first method was based on the observation that the sharpness of the iris edge varies with and without a cosmetic lens, and can be used as a measure for cosmetic contact lens detection. Their second method was based on extracting texton features from the iris. The third method utilized textural features based on co-occurrence matrices. He et al. [11] proposed to combine Adaboost learning and LBP (Local Binary Patterns) for distinguishing the textural patterns between irides with and without a cosmetic contact lens. Zhang et al. [19] suggested combining SIFT and LBP to generate a weighted LBP map, and employed an SVM classifier to distinguish iris images with and without a cosmetic lens.

Detection of non-cosmetic contact lenses is a more challenging problem than detecting cosmetic contact lenses due to the fact that non-cosmetic lenses are almost imperceptible once they are placed in the eye. In an NIR image, most non-cosmetic contacts cannot be easily discerned even by the human eye. However, the lens border is partly visible. Since non-cosmetic contact lenses do not obscure the iris, the techniques that are typically used to detect fake iris patterns cannot be trivially used for the detection of non-cosmetic contact lenses. Detection of non-cosmetic lenses as well as cosmetic lenses has been attempted by Kywe et al [20]. Their technique proposes using a thermal camera to measure the temperature decrease on the surface of the eye resulting from evaporation of water during blinking. Kywe et al. hypothesize that the magnitude of temperature decrease on an eye without a lens differs from that of an eye with a lens. They correctly classified 26 of the 39 total subjects in their dataset, resulting an accuracy of $66.7 \%$. However, their results were highly dependent on the humidity and temperature of the environment. Consequently, the performance of their algorithm decreased to $50 \%$ during the summer or in high humidity.

\subsection{Proposed Method}

Classical edge detection methods, which are based on detecting sharp changes in the pixel intensity, appear to perform poorly on detecting non-cosmetic soft contact lenses. The poorly visible thin lens border has a high probability of being missed, whereas occlusions and non-uniform illumination on the image have a high probability of being detected. Further, factors such as level of visibility, location of the lens boundary, occlusions, and illumination vary across images, thereby complicating parameter selection for edge detection methods. Figure 2.7 illustrates this quandary 
by applying a canny edge detector to an ocular image hosting a non-cosmetic soft contact lens. Note that the lens boundary is either barely visible or hardly distinguishable in the edge images.

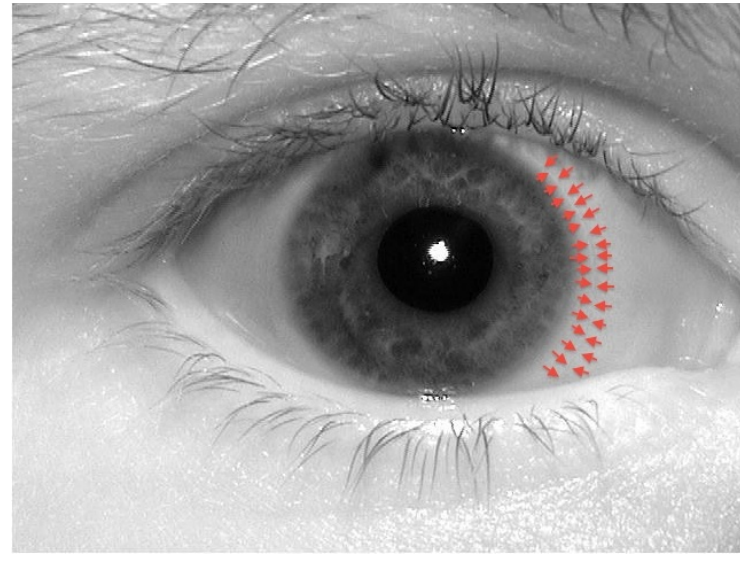

(a)

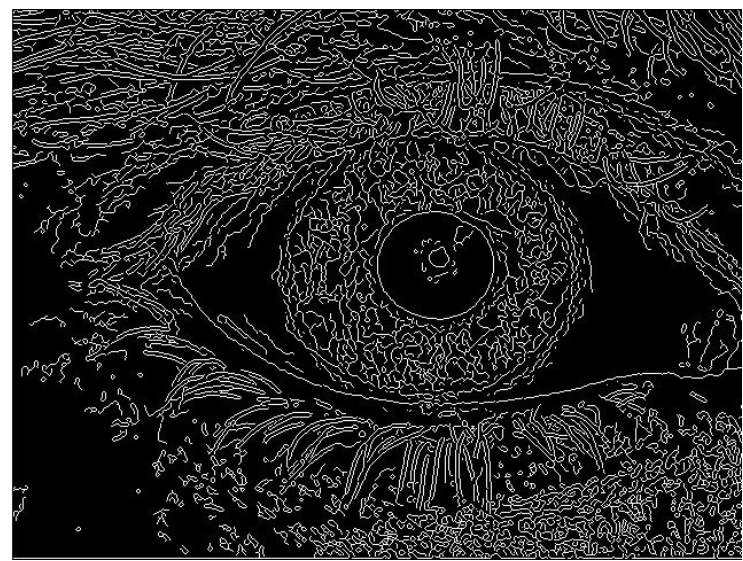

(c)

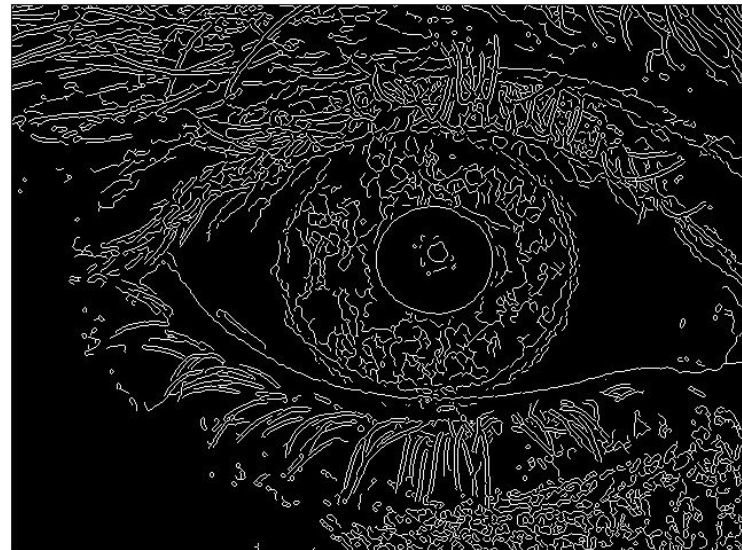

(b)

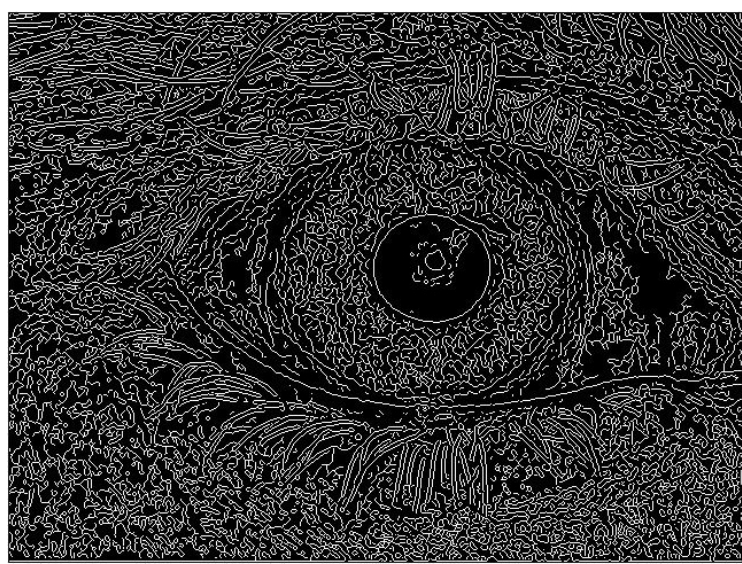

(d)

Figure 2.7: The futulity of applying a canny edge operator to an NIR ocular image in order to detect contact lenses. The original image is given in (a). (b) Detected edges when threshold $=$ $\{0.0024,0.06\}$ and $\sigma=\sqrt{2}$. (c) Detected edges when threshold $=\{0.0016,0.04\}$ and $\sigma=\sqrt{2}$. (d) Detected edges when threshold $=\{0.0008,0.02\}$ and $\sigma=\sqrt{2}$.

Since global contrast evaluation methods may often elicit insufficient or redundant information while failing to extract the lens boundary, we propose to utilize local intensity profiles of the pixels. The proposed method begins with image denoising and segmentation of the iris. The purpose of denoising is to eliminate the noise and to better observe the pixel intensity profiles without los- 
ing the necessary data corresponding to the lens boundary. Image denoising is performed using a Gaussian filter with a standard deviation of 0.5. The image smoothing is followed by the segmentation of the iris. The iris is segmented through the use of the method provided by Masek et al. [21] and the coordinates of the outer limbus boundary is stored in polar form. Figure 2.8 illustrates the preprocessing of a sample image from the ICE 2005 database.

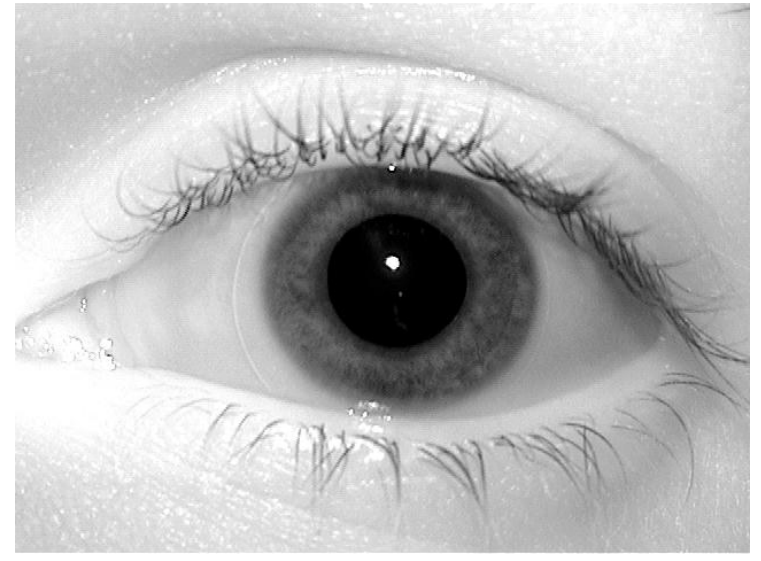

(a)

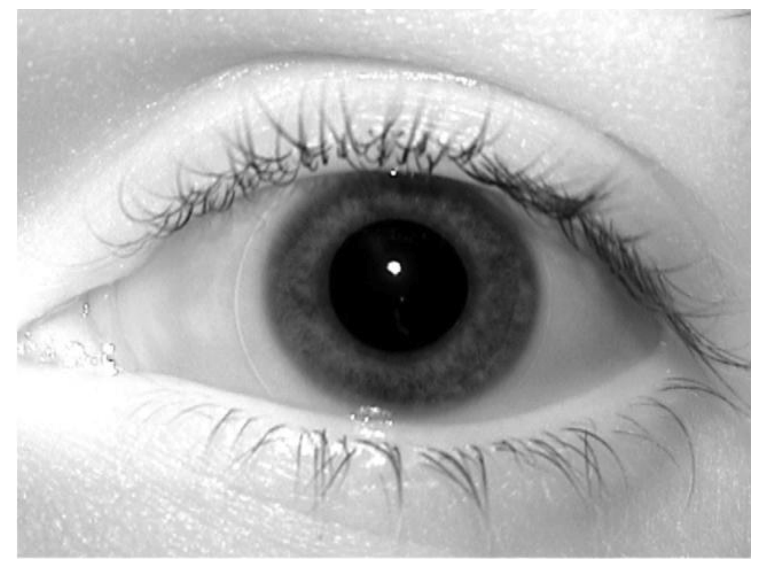

(b)

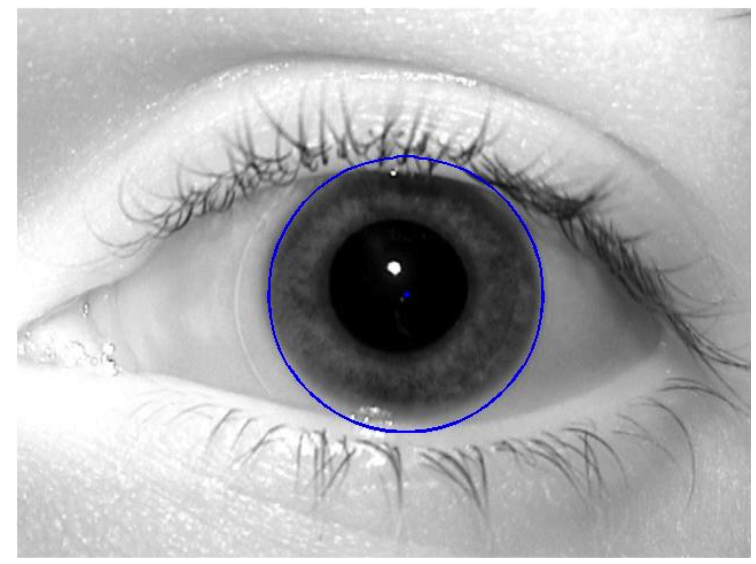

(c)

Figure 2.8: Preprocessing the input image. (a) Original eye image, whereas (b) Image after denoising. (c) Segmentation of outer iris boundary.

Next, the algorithm traverses the annular region in the vicinity of this boundary twice. During each traversal, the segment of the sclera in the interval $[\pi / 4,3 \pi / 4]$ and $[-3 \pi / 4,-\pi / 4]$ with respect to the iris center, is covered (see Figure 2.9). 


\subsubsection{Traversal \#1}

In this step, a set of line segments are defined on the vicinity of the iris boundary in the radial direction at angular intervals of $1^{\circ}$. These segments are directed away from the iris center and are orthogonal to the tangent of the iris boundary at that particular angle. Each segment begins at an offset of 5 pixels from the limbus boundary, points in the radial direction away from the iris center, and is 30 pixels long. The length of these line segments was dictated by the following considerations:

- The line segment has a high possibility of intersecting the lens boundary in with-lens images.

- Only a small number of pixels from the inside of the iris is included.

- Pixels corresponding to eyelids or eyelashes are excluded to a great extent.

Figure 2.9 demonstrates the target region covered by the line segments.

On each line segment, the pixels with the largest intensity values are defined as largest-intensitypixels. Figure 2.10 illustrates the largest-intensity-pixels in green. Once such pixels are identified, they are grouped in clusters. The clustering process has three constraints:

- There has to be a minimum number of adjacent points, whilst traversing in the angular direction, in each cluster. This minimum is denoted by $\alpha$.

- The points within a cluster cannot exceed a certain maximum distance in the radial direction. This maximum is denoted by $\tau$.

- The points in a cluster should not include any pixels located on the outer end of a line segment.

The two parameters, $\alpha$ and $\tau$, are selected according to the characteristics of the images in the database. The third constraint avoids selecting pixels whose high intensity values are due to illumination from the light source instead of the lens boundary. In such cases, the pixel intensity usually increases monotonically when traversing in one direction. Omitting those line segments that have the brightest pixel in the outer end is found to be an easy-to-implement solution, resulting in better performance. Figure 2.11(a) highlights the extracted clusters in yellow. Here $\alpha=7$ and $\tau=2$. 


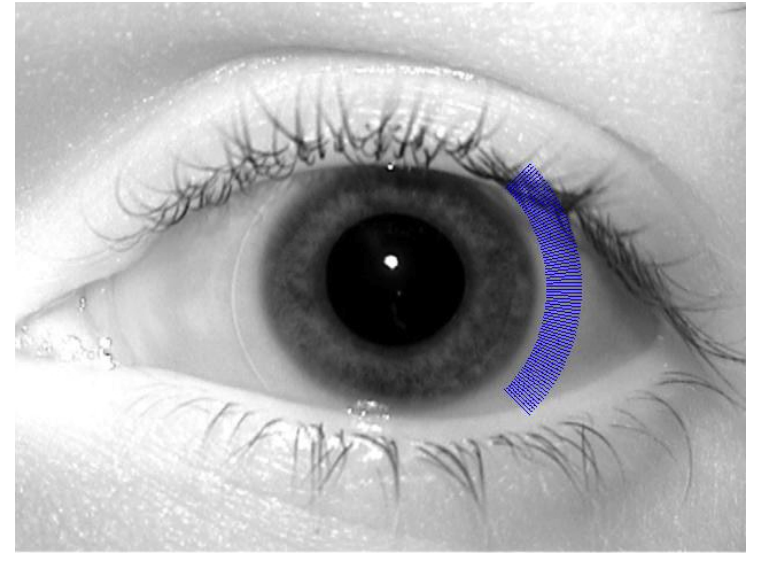

(a)

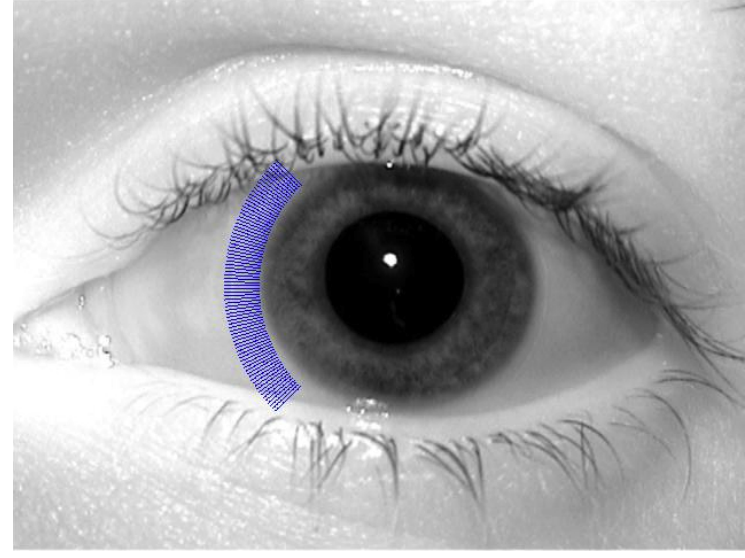

(b)

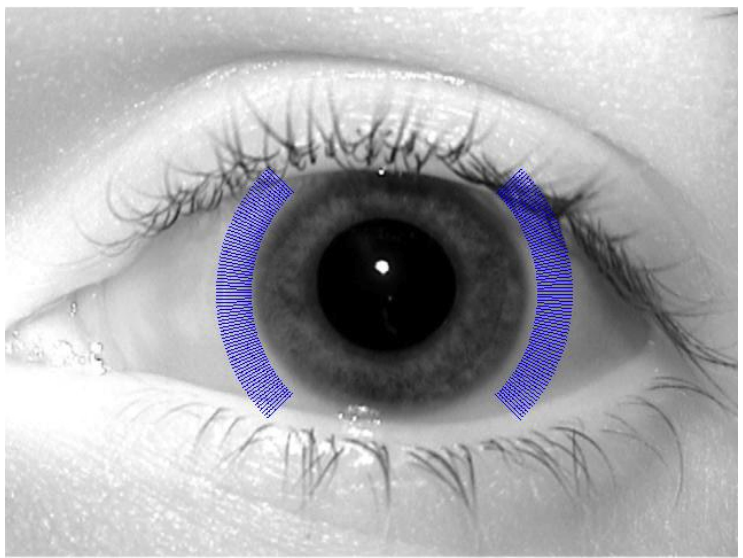

(c)

Figure 2.9: Angular region considered for detecting the boundary of the lens. (a) Line segments extracted in the angular region $[\pi / 4$ and $3 \pi / 4]$. (b) Line segments extracted in the angular region $[-3 \pi / 4$ and $-\pi / 4]$. The union of the angular regions considered in (a) and (b) can be seen in (c).

\subsubsection{Traversal \#2}

After extracting the clusters, the algorithm revisits and evaluates the extracted clusters in the radial direction in this stage. To ensure that the lens boundary is detected, an additional constraint is specified at this point: the pixels in each cluster are expected to have larger mean intensity values than the pixel sets that are in their vicinity. To compare the mean intensity values of a cluster and the surrounding pixel sets, 2 reference point sets are formed near the cluster (one on either side). Each reference point set consists of pixels at a distance of 10 units from the cluster in the radial 


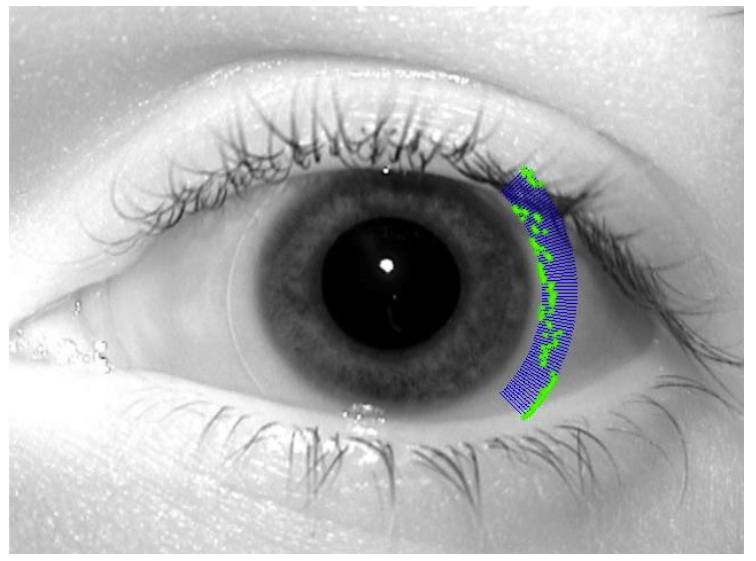

(a)

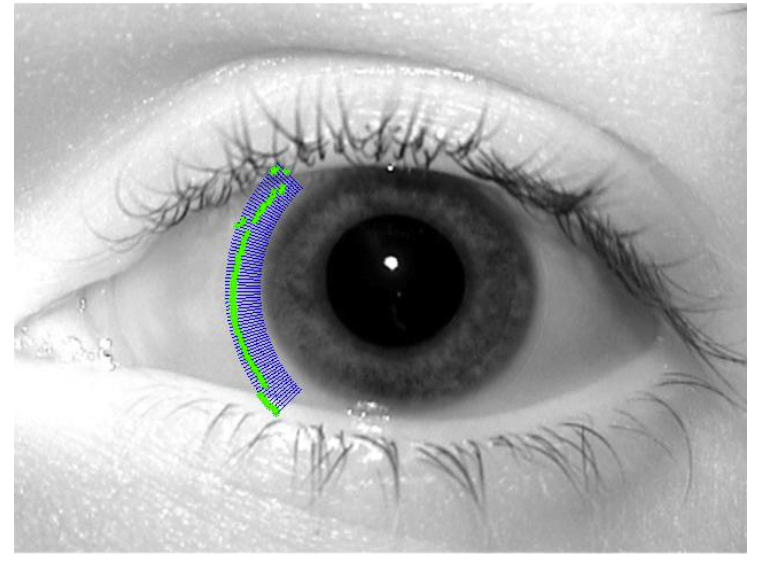

(b)

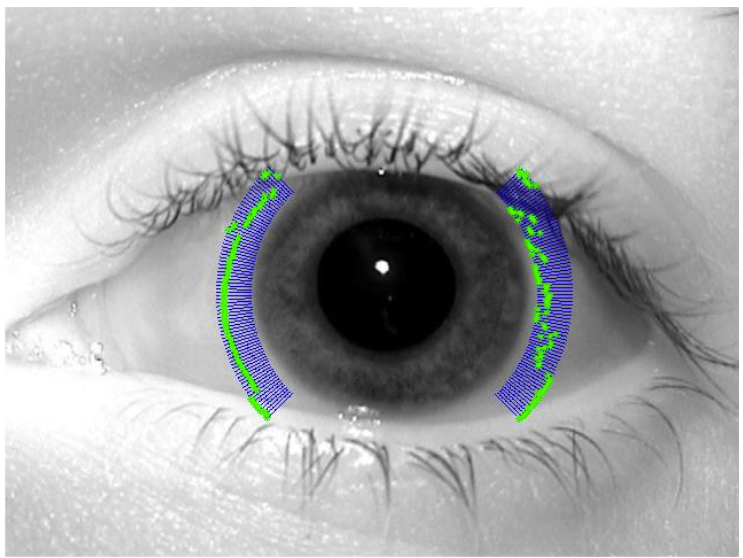

(c)

Figure 2.10: Largest intensity pixels marked. (a) Largest intensity pixels on line segments in the angular region $[\pi / 4,3 \pi / 4]$. (b) Largest intensity pixels on line segments in the angular region $[-3 \pi / 4$ and $-\pi / 4]$. (c) Union of the angular regions considered in (a) and (b).

direction. Then the mean intensity value of the cluster is compared to the mean intensities of these point sets. If a cluster has a smaller mean intensity than the mean intensities of the point sets, that cluster is deleted. Figure 2.11(b) indicates how the reference cluster sets are generated (reference set pixels are marked in black).

Eventually, if there are any clusters left, then it is concluded that the image is a with-lens image; otherwise (i.e. if no clusters are formed or all clusters are deleted), the image is classified as a without-lens image. Figure 2.11(c) illustrates the remaining valid cluster in red. The details 
of the proposed algorithm is summarized in the form of a pseudo-code in Algorithm 1.

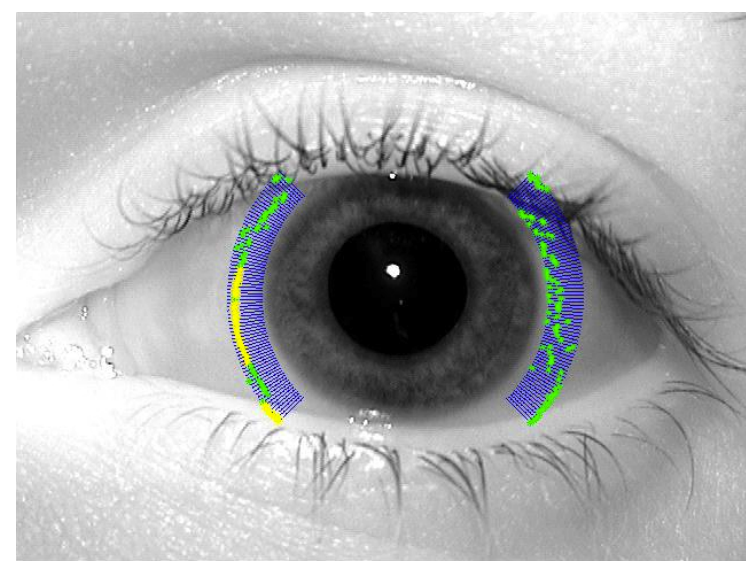

(a)

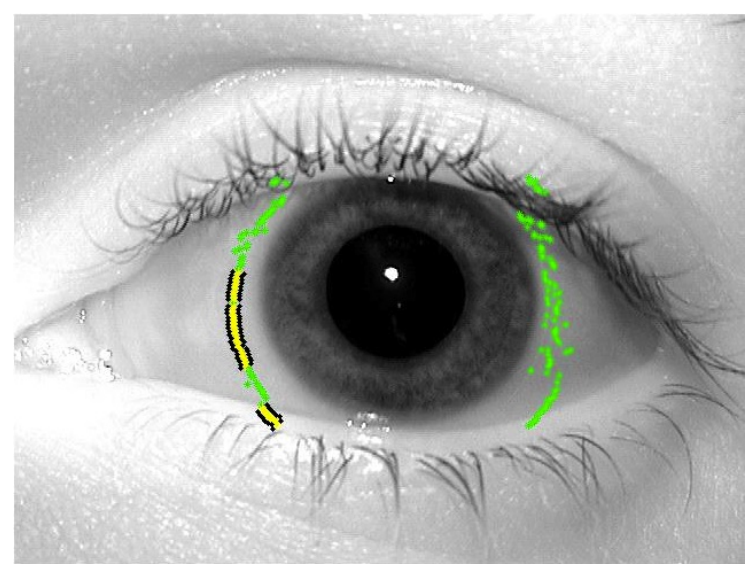

(b)

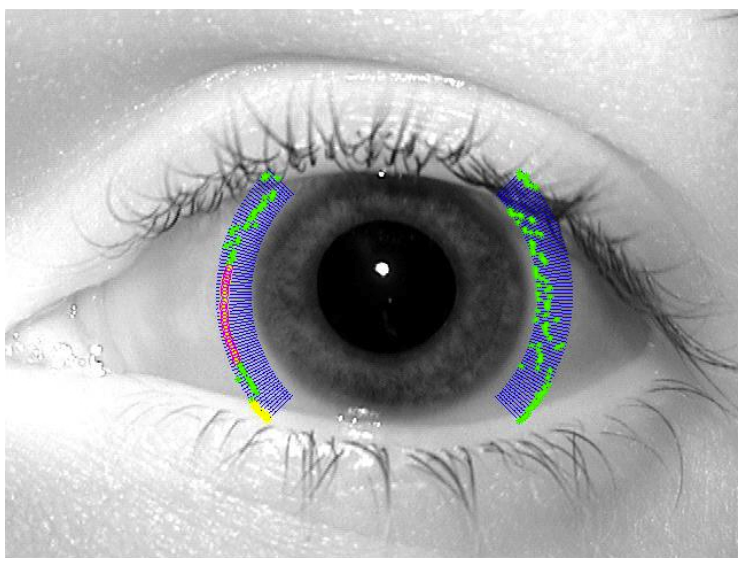

(c)

Figure 2.11: Clustering process. (a) Output the initial clustering process. Largest intensity pixels are illustrated in green and initial clusters are illustrated in yellow. (b) Reference cluster sets are illustrated in black. (c) The one remaining cluster is illustrated in red. 


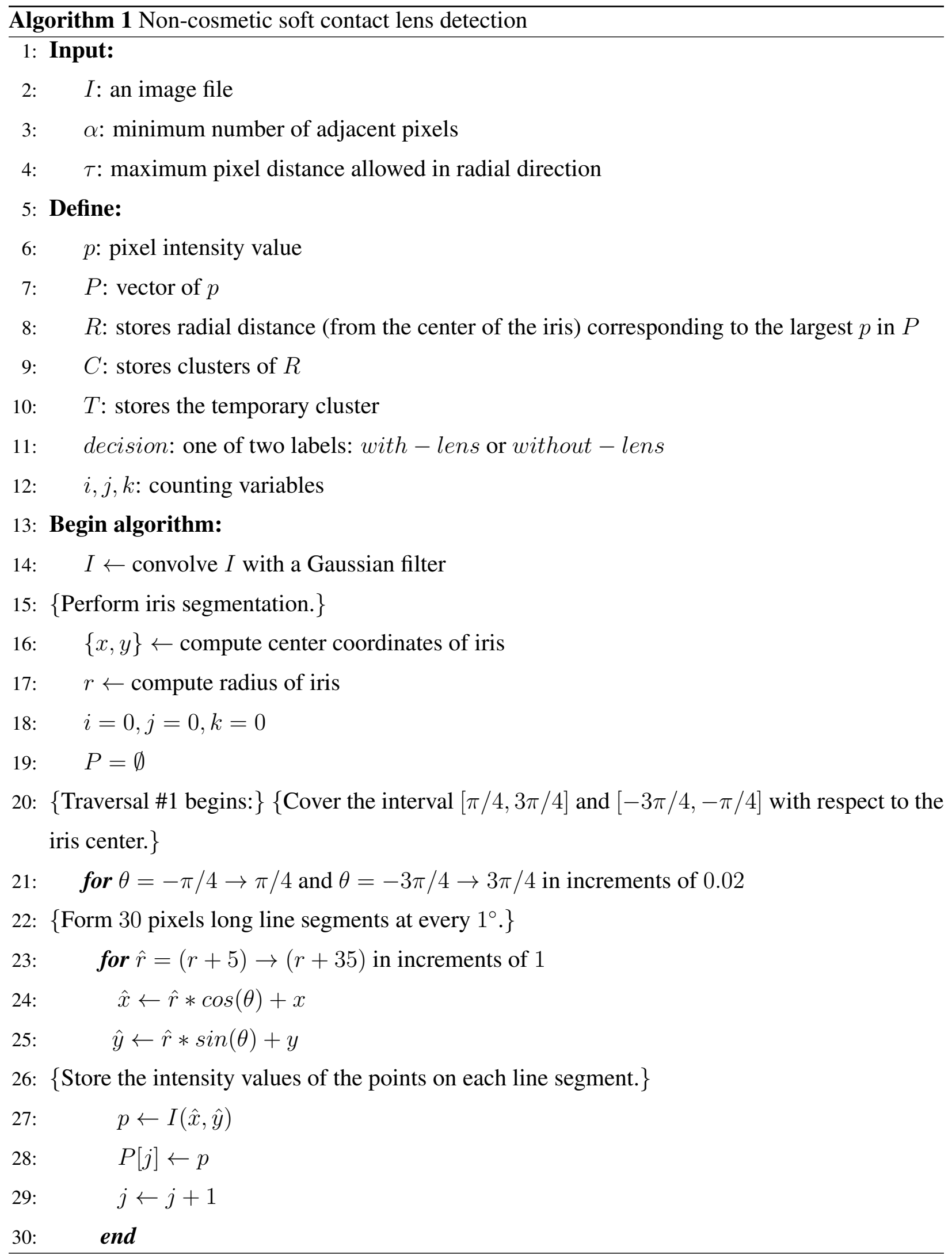


31: $\{$ Store the largest intensity value on each line. $\}$

32: $\quad R[i]=\hat{r}$ of the largest $p$ in $P$

33: $\quad i \leftarrow i+1$

34: $\quad$ end

35: $\quad T=$ null

36: $\quad \min R=+\infty$

37: $\max R=-\infty$

38: $\quad$ forEach $R[i]$ in $R$

39: \{Skip the clusters which are located at the outer ends of the line segments.

40: $\quad$ if $R[i]==(r+35)$

41: $\quad T=$ null

42: $\quad$ else

43: $\{$ Keep track of the distances between every consecutive largest intensity pixel. $\}$

44: $\quad$ if $\min (R[i], R[i+1])<\min R$

45: $\quad \min R=\min (R[i], R[i+1])$

46: $\quad$ else if $\max (R[i], R[i+1])>\max R$

47: $\quad \max R=\max (R[i], R[i+1])$

48: $\quad$ end

49: \{If the distance between the evaluated points is at most $\tau$, include the evaluated points in the cluster. $\}$

50: $\quad$ if $\operatorname{abs}(\max R-\min R) \leq \tau$

51: $\quad$ insert $R[i]$ and $R[i+1]$ into $T$

52: $\{$ Else if the distance between the evaluated points is greater than $\tau$, complete the cluster without including them.\}

53: else

54: $\quad$ if $T$ has at least $\alpha$ instances

55: $\quad C[k] \leftarrow T$

56: $\quad T=$ null

57: $\quad k \leftarrow k+1$

58: $\{$ If the cluster has less than $\alpha$ points, do not further consider this cluster. $\}$

59: $\quad$ else

60: $\quad T=$ null 


61: $\quad$ end
62: $\quad$ end
63: $\quad$ end
64: $\quad\{$ Traversal $\# 2$ begins: $\}$
65: $\quad$ for $\boldsymbol{E}$ ach $C[i]$ in $C\{$ Compare the mean intensity of a cluster with the reference point sets
surrounding it. $\}$
66: $\quad$ mean $R \leftarrow$ mean (intensities of all pixels located at $C[i]$ )
67: $\quad$ mean $1 \leftarrow$ mean (intensities of pixels within a range of +10 pixels from $C[i]$ in the radial
direction)

68: $\quad$ mean $2 \leftarrow$ mean (intensities of pixels within a range of -10 pixels from $C[i]$ in the radial direction)

69: \{If the cluster does not have the larger mean intensity than reference point sets, delete the cluster.

70: $\quad$ if $($ mean $R<$ mean 1$)$ OR $($ mean $R<$ mean 2$)$

71: $\quad$ delete $C[i]$

72: $\quad$ end

73: $\quad$ end

74: $\{$ If there are any clusters left in the image, mark the image as with - lens image.

75: $\quad$ if $C !=\emptyset$

76: $\quad$ decision $\leftarrow$ with - lens

77: $\quad$ else

78: $\{$ Else if there are no clusters left in the image, mark the image as without - lens image. $\}$

79: $\quad$ decision $\leftarrow$ without - lens

80: $\quad$ end

81: End algorithm: return decision 


\subsection{Experimental Results}

\subsubsection{Image Level Experiments}

The proposed algorithm for non-cosmetic soft contact lens detection was evaluated on two databases: MBGC-Iris and ICE 2005. The experimental setup for both databases is similar. Prior to evaluating the detection algorithm, each image in the dataset was labeled as with-lens or withoutlens. If at least one valid cluster is detected in an image by the proposed algorithm, the image is classified as a with-lens image. If no clusters are found, the image is classified as a without-lens image. The performance of the algorithm is measured using three metrics:

- Overall classification accuracy: The fraction of with-lens and without-lens images that are correctly classified.

- True Positive Rate (TPR): The fraction of with-lens images that are correctly classified as with-lens.

- False Positive Rate (FPR): The fraction of without-lens images that are incorrectly classified as with-lens.

For the ICE 2005 database, the labels were provided by researchers from the University of Notre Dame for 1000 images: 500 were with-lens and 500 were without-lens. When $\alpha=7$ and $\tau=2$, the proposed algorithm correctly classified 360 with-lens images and 401 withoutlens images, yielding an overall classification accuracy of $76 \%$ and a TPR of $72 \%$. The resulting confusion matrix is given in Table 2.1.

Table 2.1: Confusion matrix for the image level experiment in the ICE 2005 dataset, where $\alpha=7$, $\tau=2$

\begin{tabular}{c|l|c|c|c}
\multicolumn{1}{c}{} & \multicolumn{2}{c}{ Predicted Label } \\
\cline { 2 - 4 } \multicolumn{1}{c|}{} & with-lens & without-lens & Total \\
& with-lens & 360 & 140 & 500 \\
\cline { 2 - 4 } & & 99 & 401 & 500 \\
\cline { 2 - 4 } & without-lens & 99 & 541 &
\end{tabular}

MBGC Iris database consists of 1583 images. 14 low quality images were excluded from the experiments, and the remaining images were used. After visual inspection, 377 of the 1569 images 
were labeled as with-lens and 1192 were labeled as without-lens images. In an experiment, with $\alpha=6$ and $\tau=2,265$ of the 377 with-lens images and 783 of the without-lens images were correctly classified, yielding an overall accuracy of $66.8 \%$ with a TPR of $70.3 \%$. The resultant confusion matrix is shown in Table 2.2.

Table 2.2: Confusion matrix for the image level experiment in the MBGC Iris dataset, where $\alpha=6, \tau=2$.

\begin{tabular}{c|l|c|c|c}
\multicolumn{1}{c}{} & \multicolumn{3}{c}{ Predicted Label } \\
\cline { 2 - 4 } & with-lens & without-lens & Total \\
Actual Label & with-lens & 265 & 112 & 377 \\
\cline { 2 - 4 } & without-lens & 409 & 783 & 1192 \\
\cline { 2 - 4 } & \multicolumn{2}{c|}{ Total } & 674 & 895
\end{tabular}

To better understand the effect of the parameters, we repeated the experiments for different $\alpha$ and $\tau$ values using the same image sets from the ICE 2005 and MBGC Iris databases, and derived the Receiver Operating Characteristics (ROC) curves. The x-axis of the ROC curve corresponds to the FPR, and the y-axis corresponds to the TPR.

Using the results shown in Table 2.1, FPR is calculated as 0.20 and TPR is calculated as 0.72 when $\alpha=7$ and $\tau=2$ in the ICE 2005 dataset. Figure 2.12 shows the ROC curve at various $\alpha$ values when $\tau$ is fixed at 2. Figure 2.13 shows the ROC curve at various $\tau$ values when $\alpha$ is fixed at 7.

Figures 2.14 and 2.15 show the results of the experiments conducted on the MBGC Iris dataset. Figure 2.14 shows the ROC curve for different $\alpha$ values with $\tau=2$, whereas Figure 2.15 shows the ROC curve for different $\tau$ values with $\alpha=6$. At $\alpha=6$ and $\tau=2$, FPR is calculated as 0.34 and TPR is calculated as 0.70 , from Table 2.2.

The results of this analysis suggest that $\alpha$ and $\tau$ are tunable parameters that have to be adjusted. And, a future research direction can be adapting $\alpha$ and $\tau$ automatically to the dataset. 


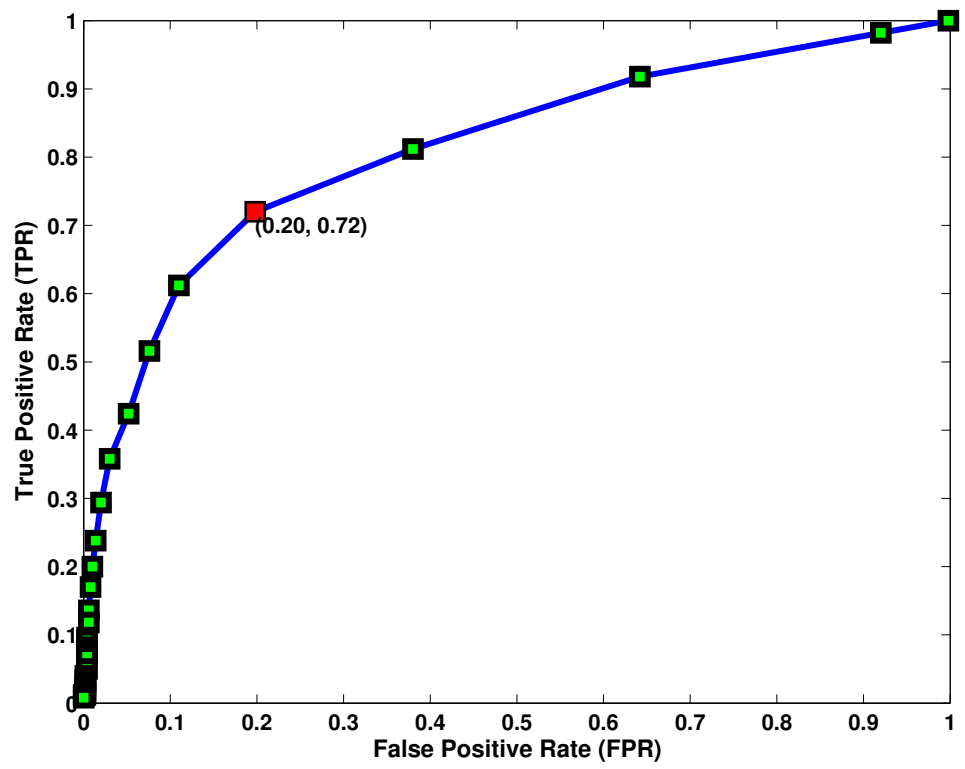

Figure 2.12: ROC curve for the image level experiment in the ICE 2005 dataset, based on varying $\alpha$ from 1 to 40 at $\tau=2$.

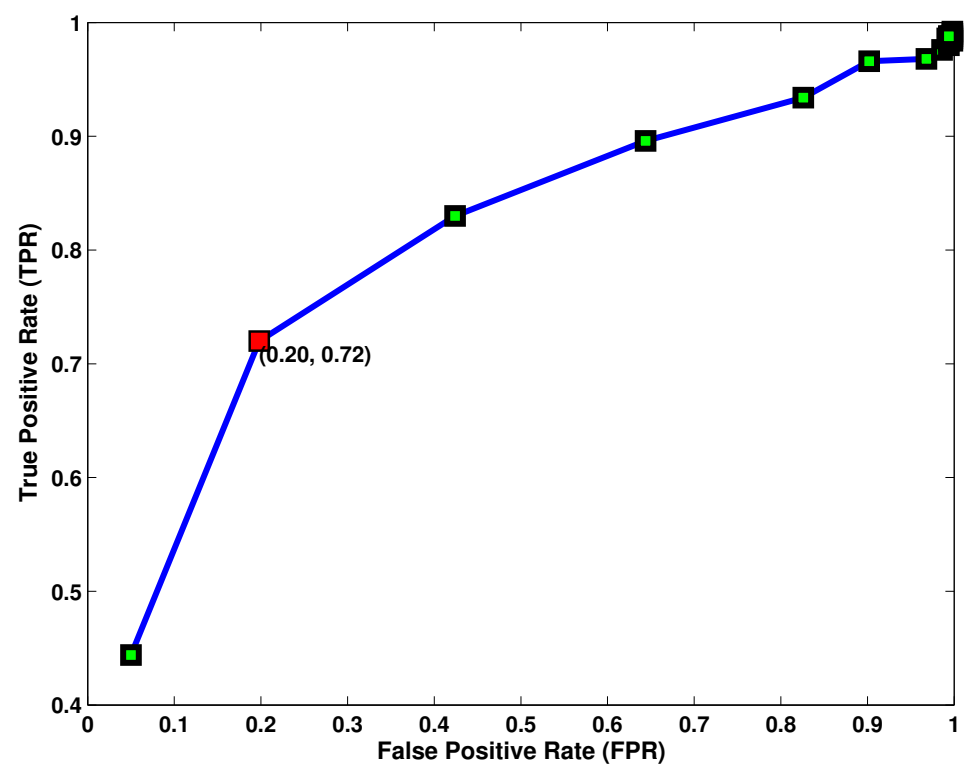

Figure 2.13: ROC curve for the image level experiment in the ICE 2005 dataset, based on varying $\tau$ from 1 to 20 at $\alpha=7$. 


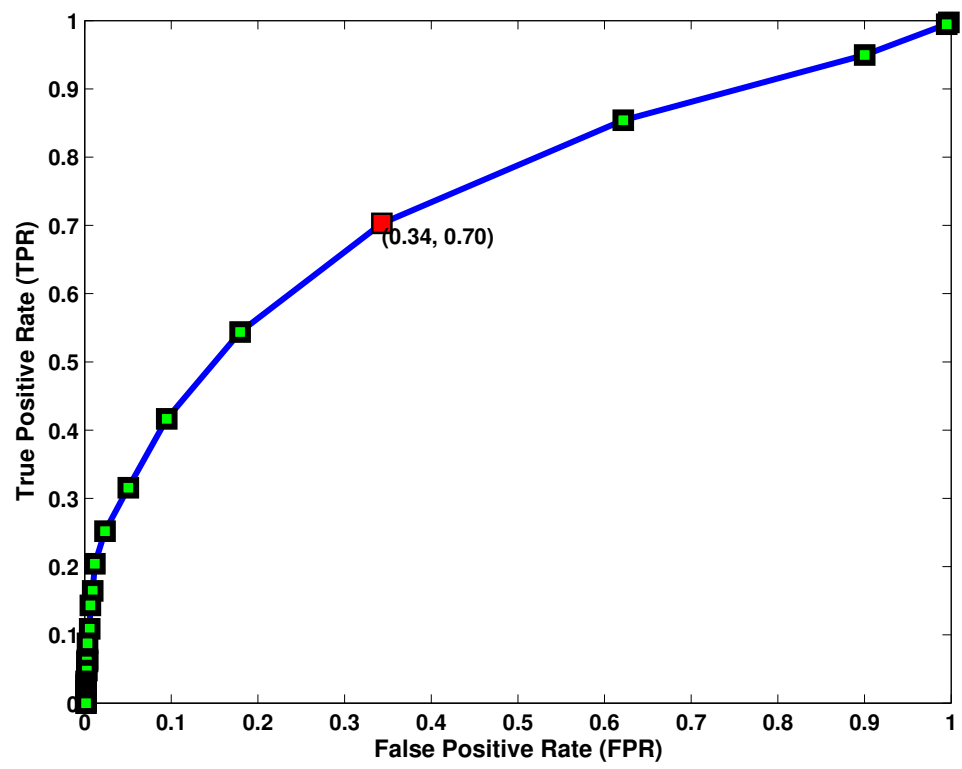

Figure 2.14: ROC curve for the image level experiment in the MBGC Iris dataset, based on varying $\alpha$ from 1 to 40 at $\tau=2$.

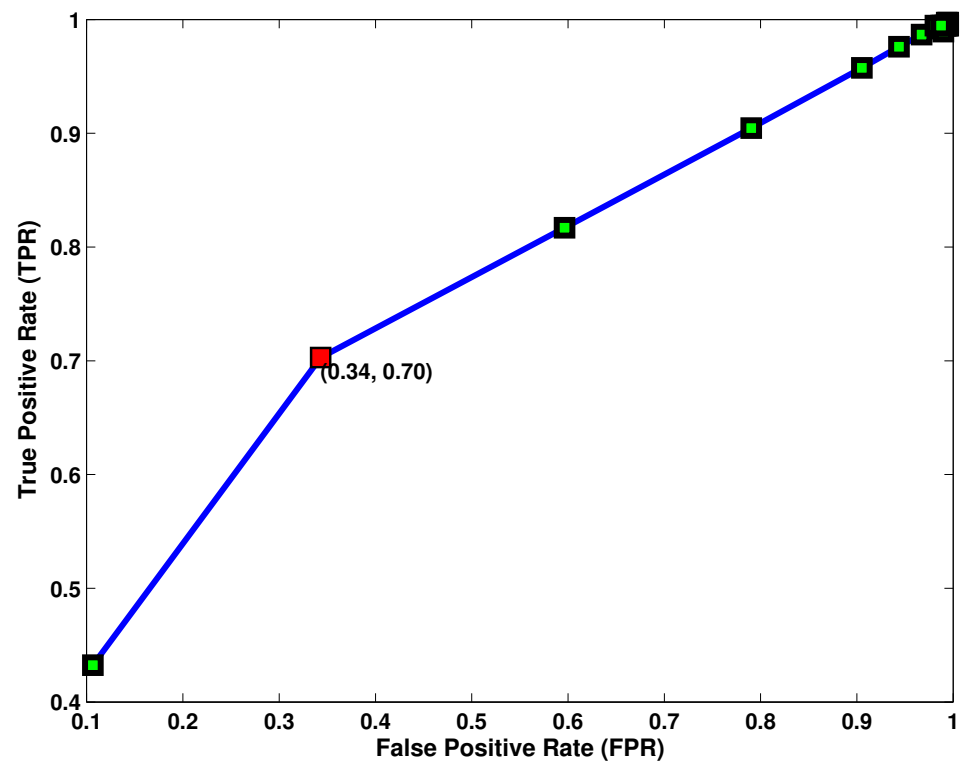

Figure 2.15: ROC curve for the image level experiment in the MBGC Iris dataset, based on varying $\tau$ from 1 to 20 at $\alpha=6$. 


\subsubsection{Subject Level Experiments}

During the preparation of the datasets for our experiments, we realized that the ground truth may not be completely accurate since the presence of a lens cannot be always easily discerned. Inconsistent illumination and camera defocus are dominant factors that can hide the lens boundary or create an illusion of a lens, thereby challenging the labelling process. Three such examples are shown in Figure 2.16.

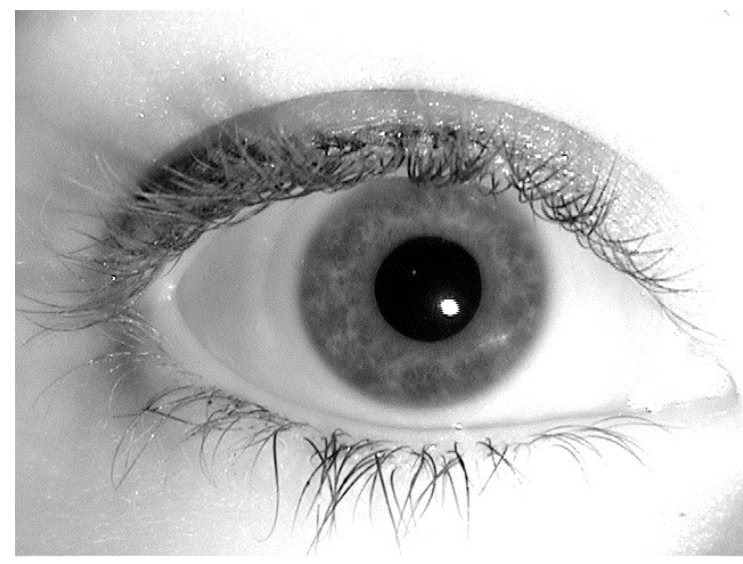

(a)

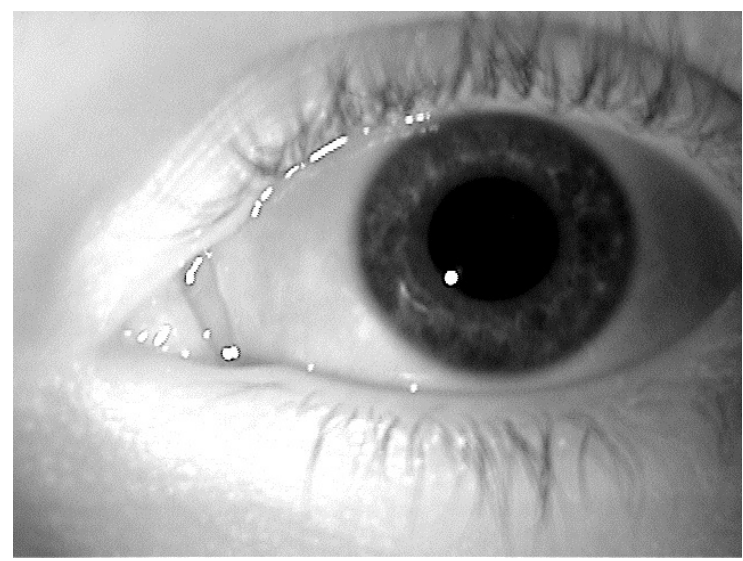

(b)

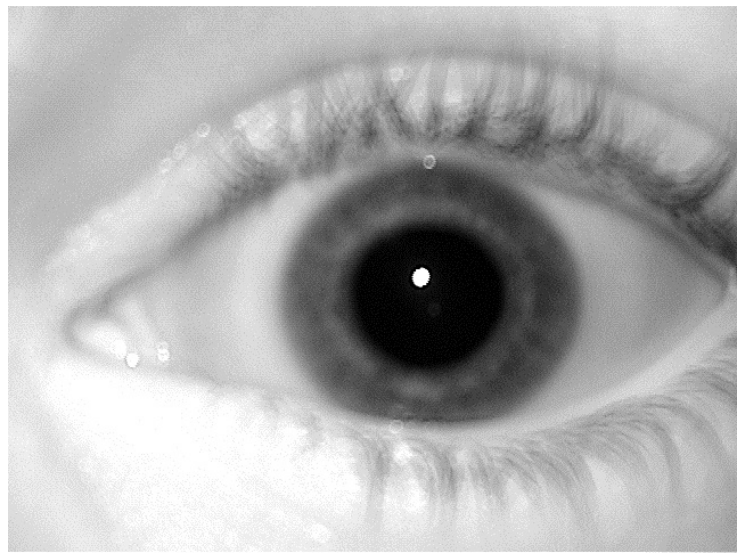

(c)

Figure 2.16: Effects of inconsistent illumination and camera defocus. (a) A case of inconsistent illumination, where the lens boundary is faintly visible in a with-lens image. (b) A case of inconsistent illumination, where a lens illusion is present in a without-lens image. (c) A case where the lens boundary is not distinguishable in a with-lens image due to defocus blur. 
Table 2.3: Confusion matrix for the subject level experiment in the ICE 2005 dataset where $\alpha=9$, $\tau=4$.

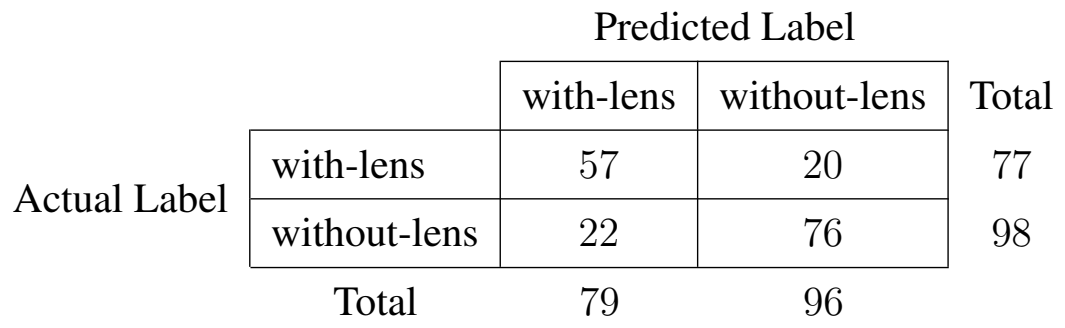

To compensate for this subjectivity, we conducted an additional group of experiments. We first selected only those subjects that have 3 or more sample images. For each of these subjects, the first 3 images were selected. Next, the manually assigned labels associated with these 3 images were examined. Majority voting was then used to re-assign labels to the 3 images. That is, if a subject had 2 or more with-lens images, then the subject was classified as a with-lens subject; else, the subject was classified as a without-lens subject. In this set of experiments, the TPR defines the fraction of correctly classified subjects out of all the with-lens subjects in the experiment set, whereas the FPR defines the fraction of incorrectly classified subjects out of all the without-lens subjects in the experiment set.

There are 175 subjects in the ICE 2005 database who have 3 or more images. 98 of these were deemed to be without-lens subjects. 77 subjects, on the other hand, were deemed to be with-lens subjects. The confusion matrix of the proposed method, with $\alpha=9$ and $\tau=4$, is given in Table 2.3. The algorithm correctly classified 57 out of 77 with-lens subjects and 76 out of 98 without-lens subjects, yielding an overall classification accuracy of $76 \%$ and a TPR of $74 \%$.

The MBGC Iris database consists of 1583 images from 98 subjects. 73 of them were deemed to be without-lens subjects. The remaining 25 subjects were deemed to be with-lens subjects. All 25 subjects have with-lens images belonging to both eyes. 71 out of 73 without-lens subjects have without-lens images corresponding to both eyes, and the remaining two subjects have images only for one eye.

The confusion matrix resulting from the experiment with 50 with-lens and 144 without-lens eyes is shown in Table 2.4. In this experiment $\alpha=9$ and $\tau=4.35$ out of 50 with-lens subjects and 113 out of 144 without-lens subjects were correctly classified, yielding an overall accuracy of $76.3 \%$ with a TPR of $70 \%$. 
Table 2.4: Confusion matrix for the subject level experiment in the MBGC Iris dataset where $\alpha=9, \tau=4$.

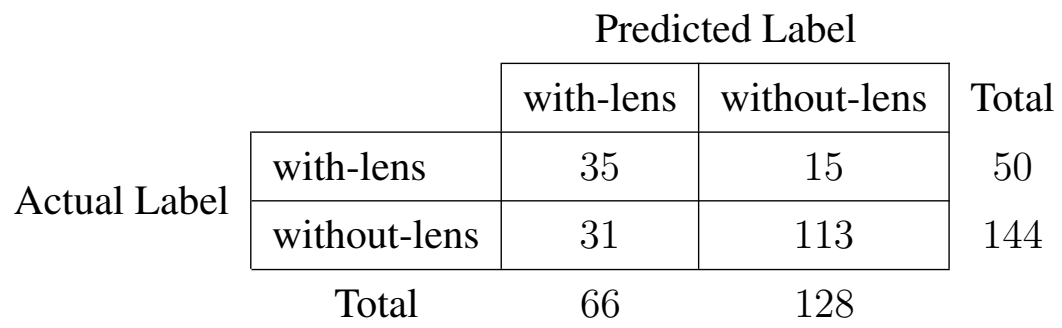

From Table 2.3, FPR is calculated as 0.22 and TPR is calculated as 0.74 when $\alpha=9$ and $\tau=4$ in the ICE 2005 dataset. Figure 2.17 shows the ROC curve for different $\alpha$ values when $\tau$ is fixed at 4. Figure 2.18 shows the ROC curve for different $\tau$ values when $\alpha$ is fixed at 9 .

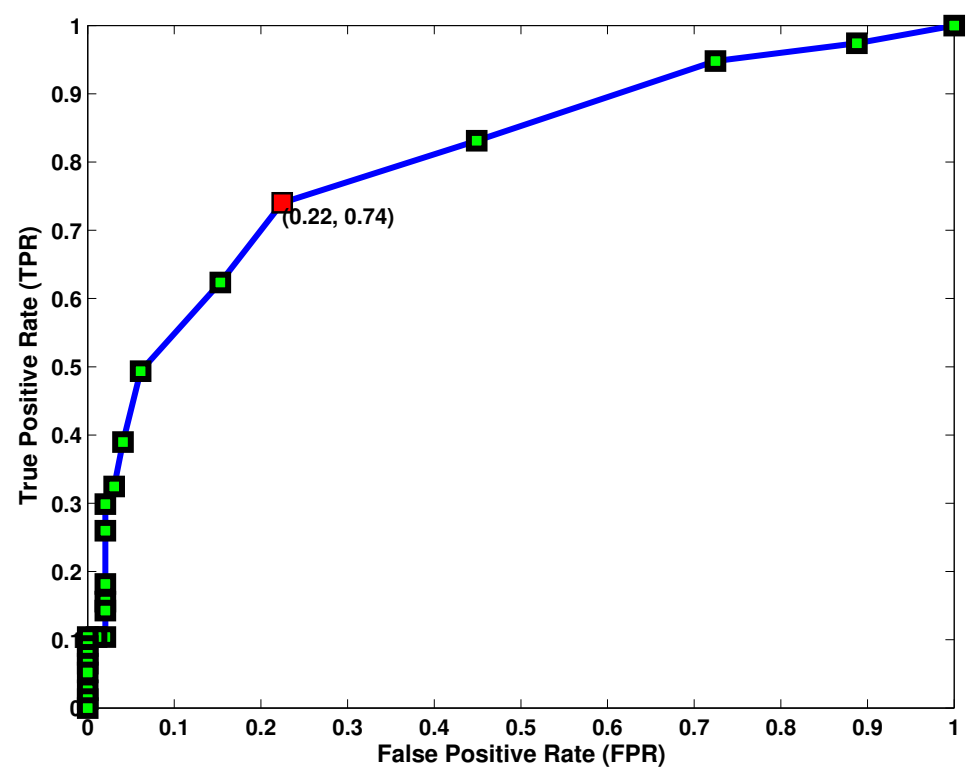

Figure 2.17: ROC curve for the subject level experiment in the ICE 2005 dataset, based on varying $\alpha$ from 1 to 40 at $\tau=4$.

From Table 2.4, FPR is computed as 0.21 and TPR is calculated as 0.70 in the MBGC Iris dataset. Figure 2.19 shows the ROC curve for various $\alpha$ values with $\tau=4$. Figure 2.20 shows the ROC curve for various $\tau$ values, with $\alpha=9$. 


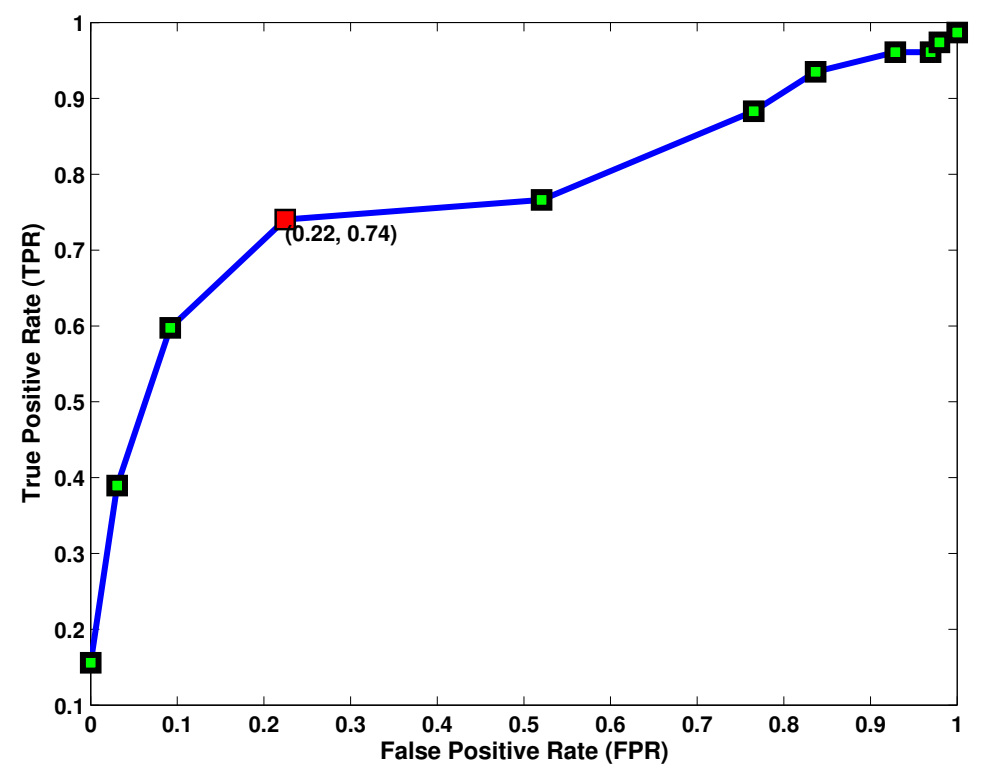

Figure 2.18: ROC curve for the subject level experiment in the ICE 2005 dataset, based on varying $\tau$ from 1 to 20 at $\alpha=9$.

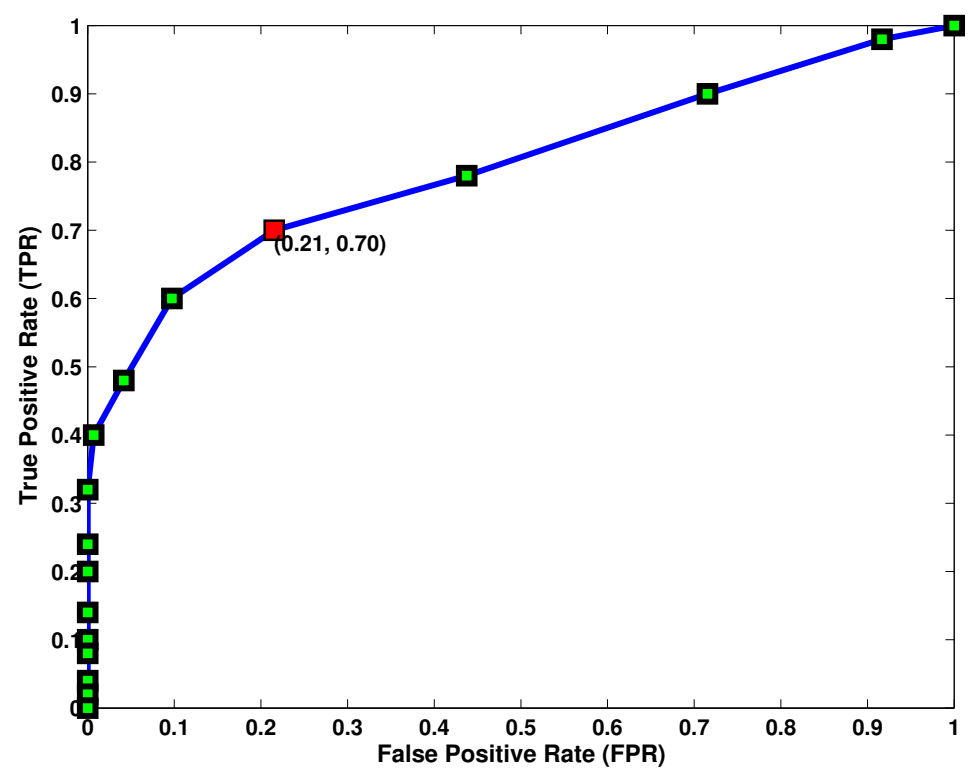

Figure 2.19: ROC curve for the subject level experiment in the MBGC Iris dataset, based on varying $\alpha$ from 1 to 40 at $\tau=4$. 


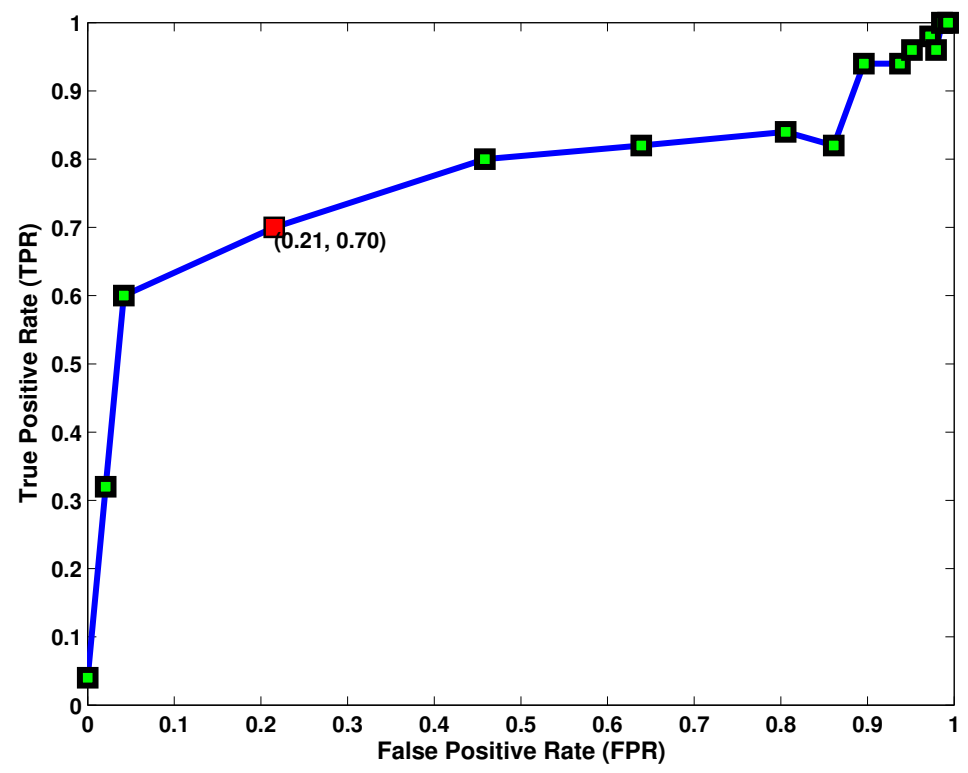

Figure 2.20: ROC curve for the subject level experiment in the MBGC Iris dataset, based on varying $\tau$ from 1 to 20 at $\alpha=9$.

\subsubsection{Challenges}

When investigating the cases where the algorithm failed, we noticed that there are several reasons impacting the performance. Consider an example from the ICE database with $\alpha=7$ and $\tau=2$. The related confusion matrix was previously shown in Table 2.1. After reviewing the 140 with-lens images which are incorrectly classified as without-lens, the following observations were made:

- In 89 images, the lens border is not significantly distinguishable from surrounding pixels. Defocus blur is also a plausible reason for failure in these cases. An example of such an image can be seen in Figure 2.21(a).

- In 34 images, although the lens boundary is significant enough, nearby pixels are brighter due to inconsistent illumination. An example demonstrating inconsistent illumination is shown in Figure 2.16(a).

- In 14 images, segmentation of the limbus boundary gave inaccurate results, thereby misleading the algorithm. An example of this case is given in Figure 2.21(c). 
- In two images, the lens is misplaced in the eye. An example case can be seen in Figure 2.21(e). The lens boundary overlaps with the limbus boundary on the lower left of the iris, and it extends far beyond the cornea on the right side of the iris.

- In one image, the lens border is completely located on the outer boundary of the line segments. This represents an outlier case, since the proposed algorithm is designed to deliberately ignore pixels that lie exactly on the outer end of the line segments.

Similarly, when examining the incorrectly classified without-lens images, it was observed that the errors are primarily due to inconsistent illumination.

- In 90 images, inconsistent illumination confuses the algorithm.

- In five images, segmentation of the limbus boundary gave inaccurate results. Figure 2.21(d) gives an example of such a case.

- In four images, the eyelids interfere with the target region and the higher pixel intensity of the skin misleads the algorithm. Figure 2.21(b) illustrates an example of this case. 


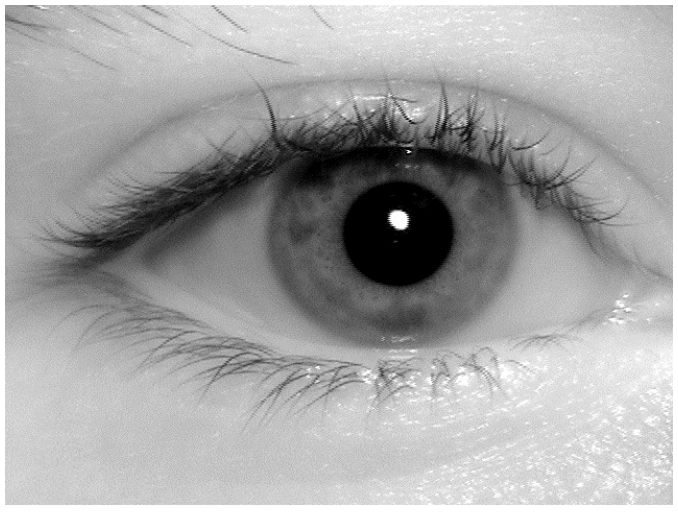

(a)

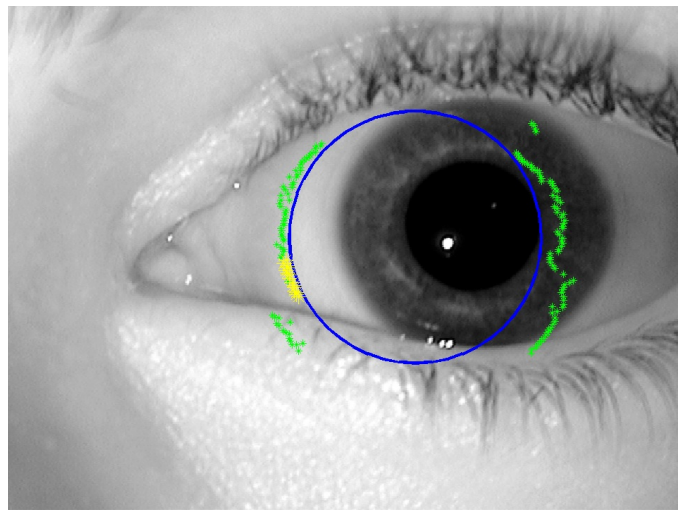

(c)

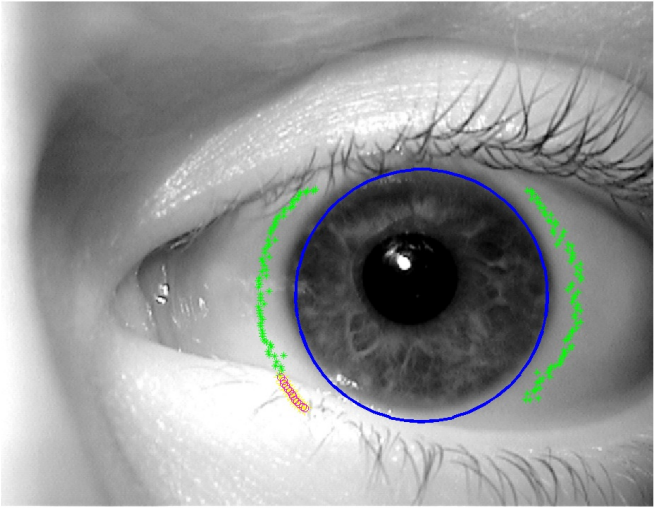

(b)

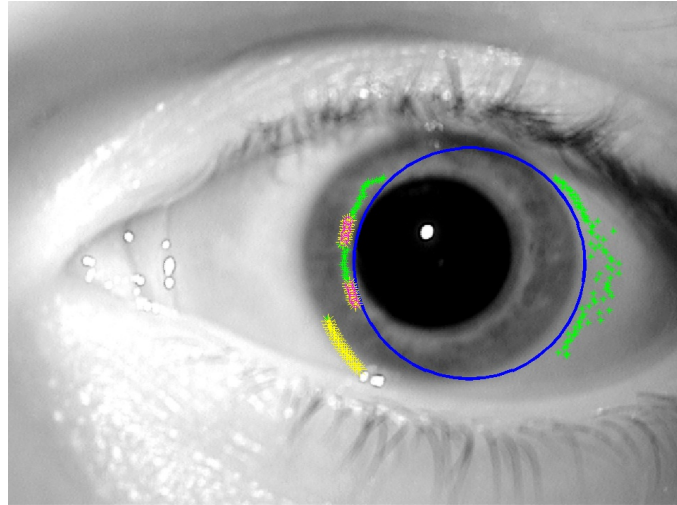

(d)

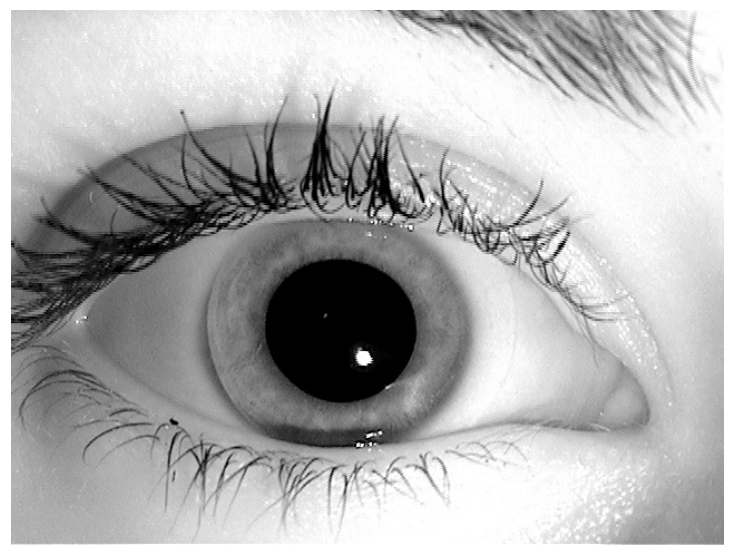

(e)

Figure 2.21: Examples of unsuccessful classification cases. (a) An example of an insignificant lens boundary. (b) An example of eyelid interference. (c) Inaccurate segmentation of the limbus boundary, which leads to misclassifying a with-lens image. (d) Inaccurate segmentation of the limbus boundary, which causes misclassification of a without-lens image. (e) An example where the lens is misplaced in the eye. 


\subsection{Summary}

In this chapter, we presented a novel method for detecting non-cosmetic contact lenses in ocular images. The proposed method identifies the points on a lens perimeter by evaluating the local intensity profiles in the vicinity of the iris. Two different set of experiments are conducted in ICE 2005 and MBGC Iris databases to evaluate the proposed model. In the image level experiments, we obtained an overall classification accuracy of $76 \%$ in ICE 2005 and $66.8 \%$ in MBGC Iris, with correct lens detection rates of $72 \%$ and $70 \%$, respectively. In the subject level experiments, we achieved an overall classification accuracy of $76 \%$ in ICE 2005 and $76.3 \%$ in MBGC Iris, with correct lens detection rates of $74 \%$ and $70 \%$, respectively. The challenges encountered in this study were mainly due to inconsistent illumination and defocus blur present in the images. 


\section{Chapter 3}

\section{Generating a Dual Identity Iris Image}

Although iris recognition is highly reliable, individuals might be able to evade iris recognition by closing their eyes or changing their gaze during image acquisition. Motion blur and occlusions are the two other factors which degrade iris recognition performance significantly. Alternatively, an evader can hide his true identity by wearing cosmetic contact lenses. However, biometric systems require subjects to be recognized as authorized individuals in order to give them access to high security areas. Hence, one of the goals in attacking biometric systems is to be recognized as someone else instead of only evading recognition. Patterned lenses can be potentially used for that purpose, by copying someone else's iris pattern onto a pair of clear (non-cosmetic) contact lens [22]. Patterned lenses yield a more advanced case of spoofing than cosmetic contact lenses, since they can cause false matches instead of failure in recognition.

Our goal is to facilitate the creation of patterned contact lenses which allows a user (source) to be recognized both as the target identity to be spoofed as well as the source itself. To achieve this goal we copy only a certain percentage of the iris pattern from the target identity. Note that in this study, we only determine the procedure to create such a lens, but do not physically generate the lens. Actual lens generation is outside the scope of this thesis. Our study copies only a part of the target iris pattern which makes this model more efficient than printing the whole iris pattern to produce a patterned contact lens. Additionally, the genuine iris pattern of the subject remains mostly perceivable, and hence this model is expected to be more difficult to detect than regular patterned contact lenses. Figure 3.1 illustrates the flow of the proposed model. Figure 3.1(a) shows the creation of the composite iris pattern. Figure 3.1(b) shows the patterned contact lens 
production, which requires additional hardware and is beyond the scope of this study.

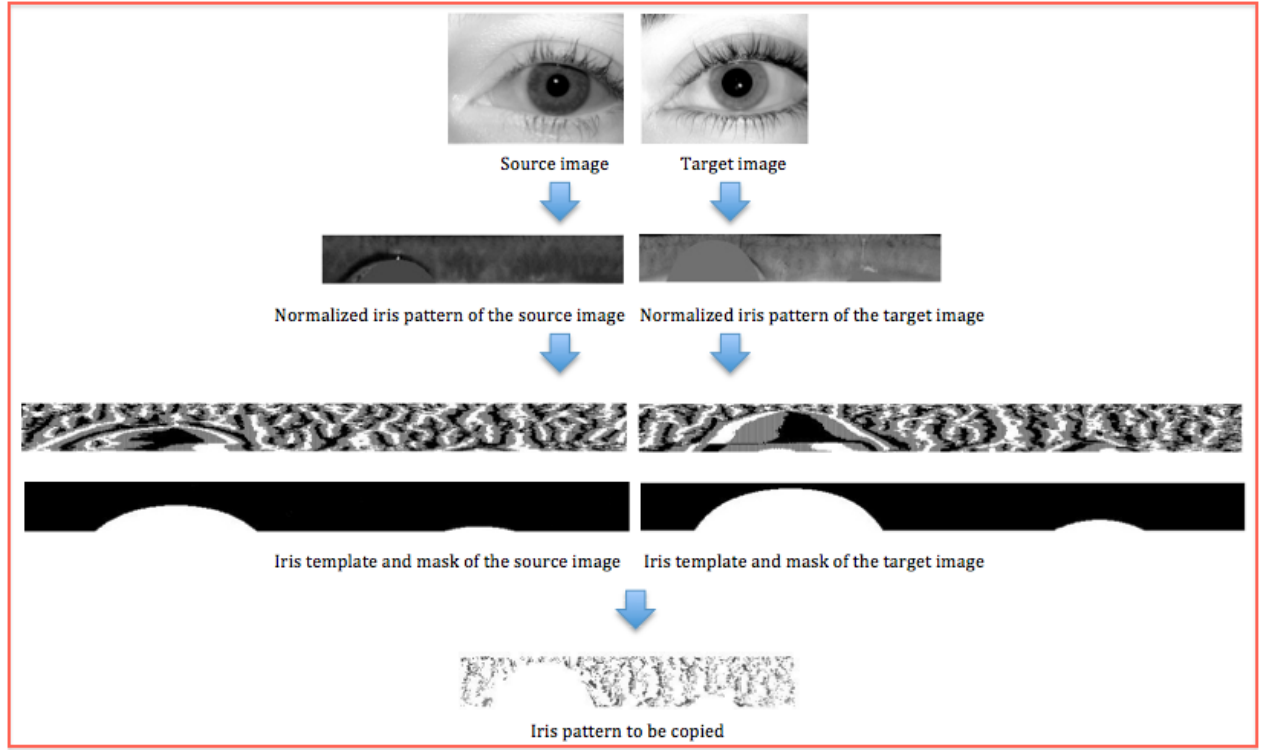

(a)

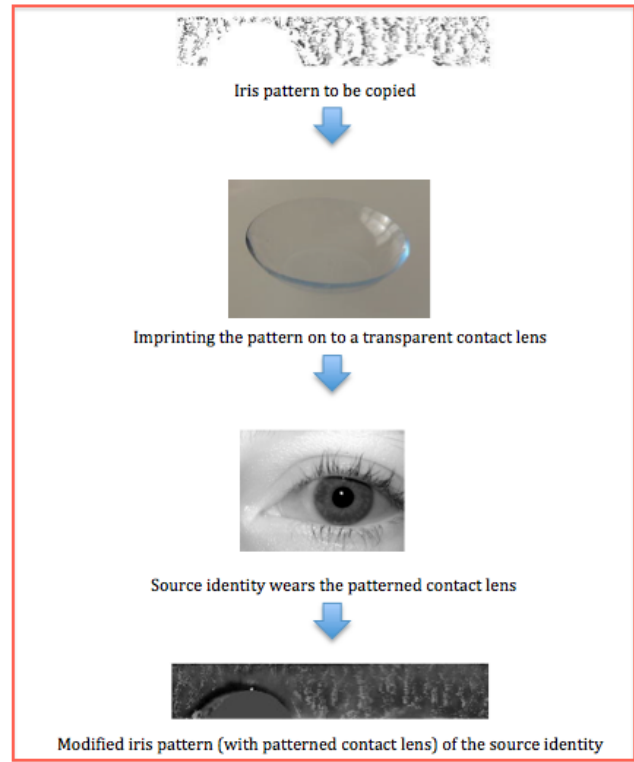

(b)

Figure 3.1: Overview of the proposed model. (a) Creation of the composite iris pattern. (b) Production of the patterned contact lens. 


\subsection{Preliminary Examples}

Assume that Figure 3.2 shows the iris pattern and iris template of a source identity and Figure 3.3 shows the iris pattern and iris template of a target identity whose iris pattern has to be copied by the source identity given in Figure 3.2. The aim is to select certain pixels from the target to be copied onto a transparent pair of lenses, so that the spoofer (source) wearing such a lens can keep sufficient information from his own iris pattern while obtaining enough information from the target identity's iris pattern. If the spoofer can host sufficient iris information related to both identities, the following constraints should hold:

- The hamming distance (HD) between the spoofer's modified iris (modified due to the placement of the patterned lens) and his genuine iris should be less than 0.32 . (This threshold is selected according to Daugman's indication that a HD $\leq 0.32$ distinguishes iris patterns with high confidence [6].)

- The hamming distance between the spoofer's modified iris and the target identity's genuine iris should be less than 0.32 .

How should the pixels be selected so that the above constraints hold? Two trivial solutions to this question are examined in Figures 3.4 and 3.5. Figure 3.4(a) is formed by stitching the first half of a source iris pattern to the second half of the target identity's iris pattern. Encoding the stitched image in Figure 3.4(a) yields the template and mask shown in Figures 3.4(b) and 3.4(c). Note that the resultant template in Figure 3.4(b) is also a combination of the first half of the template given in Figure 3.2(c) and the second half of the template given in Figure 3.3(c) with a slight difference in the middle of the image. The HD between the stitched image and the source iris pattern (Figure 3.2(b)) is 0.25 and the HD between the stitched image and the target iris pattern (Figure 3.3(b)) is 0.29 .

Figure 3.5(a) is formed by mixing the source iris pattern and the target identity's iris pattern uniformly. A column of pixels are selected from the source and target images alternatively and the resulting image is encoded to form the template and mask shown in Figures 3.5(b) and 3.5(c), respectively. The HD between the mixed image and source iris pattern (Figure 3.2(b)) is 0.27 and the HD between the mixed image and the target iris pattern (Figure 3.3(b)) is 0.24. 


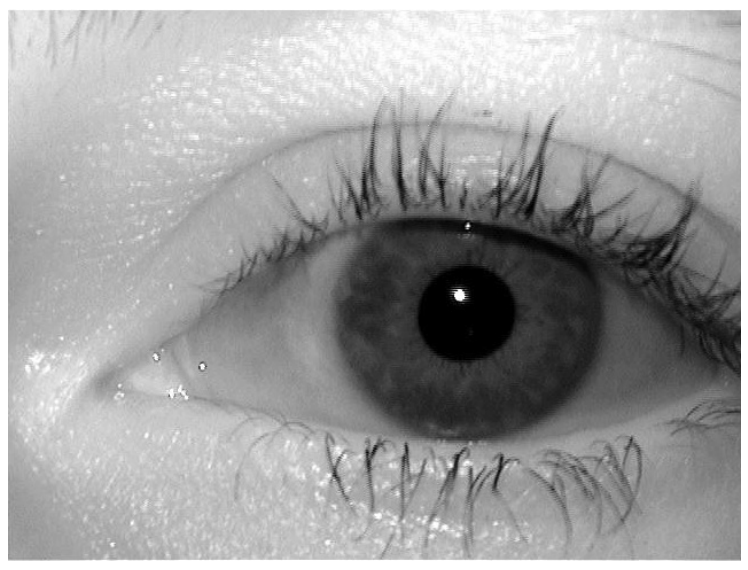

(a)

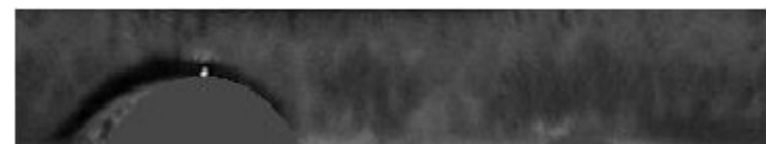

(b)

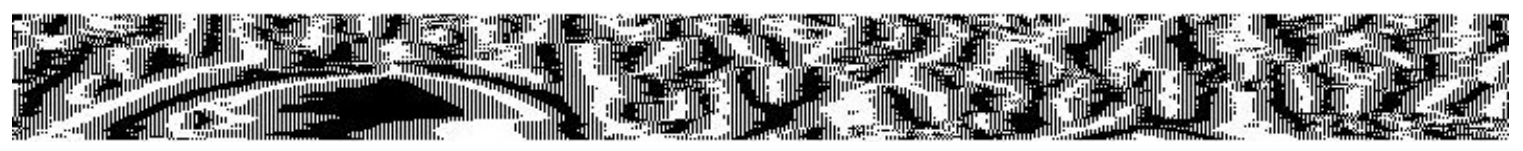

(c)

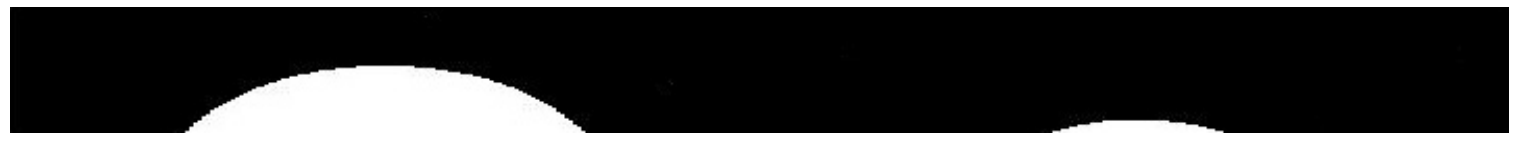

(d)

Figure 3.2: Example of a source identity's iris information. (a) Eye image of the source identity. (b) Source identity's normalized iris pattern. (c) Source identity's iris template. (d) Corresponding mask for the source identity's iris template.

As demonstrated in Figures 3.4 and 3.5, both examples yield a HD below the 0.32 threshold. However, in practice these images can be detected instantly by many spoof detection systems because of the unusual characteristics of the iris pattern. Example in Figure 3.4 contains a sharp alteration of the iris pattern and the example in Figure 3.5 contains an unnatural periodicity. More- 
over, both approaches require copying exactly $50 \%$ of the target iris pattern.

To demonstrate an alternative approach which does not contain periodicity, see Figure 3.6. A trivial solution that comes to mind would be copying pixels from one image onto the other randomly to create a mixed image. An example of this case can be seen in Figure 3.6, where 50\% of the target image's pixels are copied onto the source image. The normalized polar form and the corresponding iris template of the mixed image are given in Figures 3.6(a) and 3.6(b), respectively; neither of them carry the properties of a typical iris pattern. Yet, one might expect the HDs between the mixed image and the source and target images to be consistent with the previous examples since effectively $50 \%$ of the target image is copied onto the source image, as in the two previous cases. However, due to the nature of Gabor wavelet transform, the random mixing process results in a different output than the above cases. The HD between the mixed image and the source iris pattern (Figure 3.2(b)) is 0.40 and the HD between the mixed image and the target iris pattern (Figure 3.3(b)) is 0.43 . Hence, the resultant image fails to be recognized as either of the two identities. 


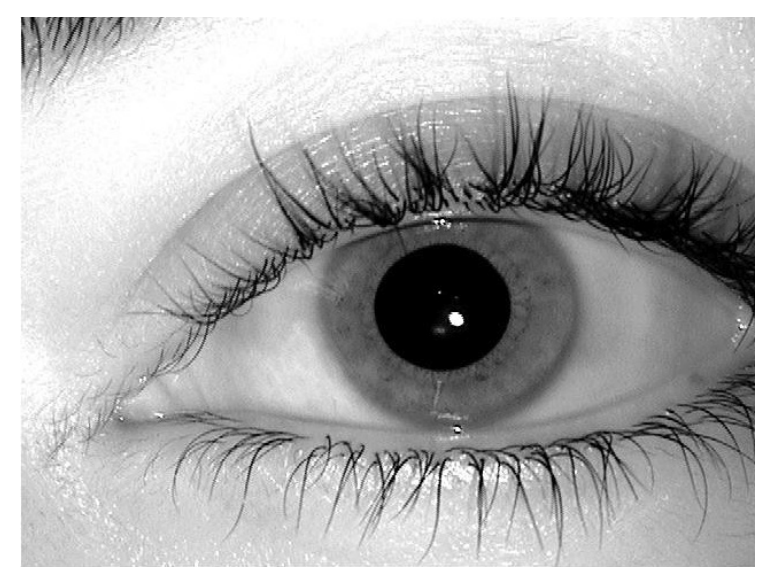

(a)

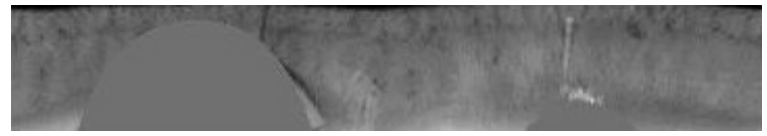

(b)

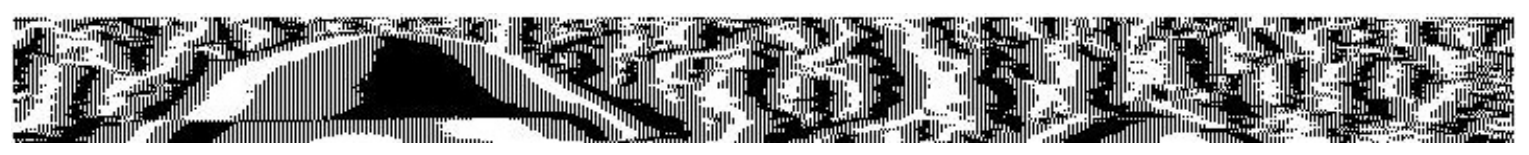

(c)

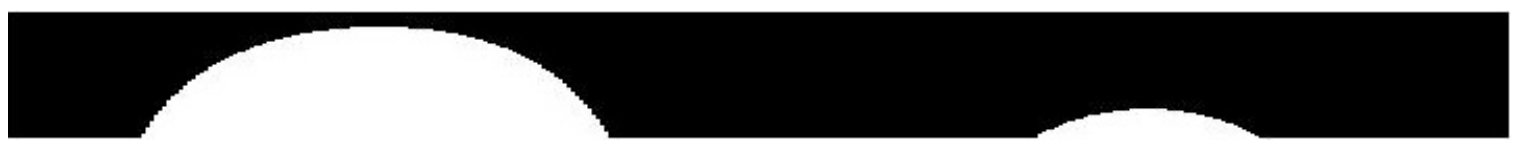

(d)

Figure 3.3: Example of a target identity's iris information. (a) Eye image of the target identity. (b) Target identity's normalized iris pattern. (c) Target identity's iris template. (d) Corresponding mask for the target identity's iris template. 


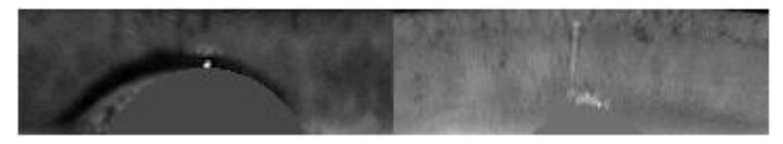

(a)

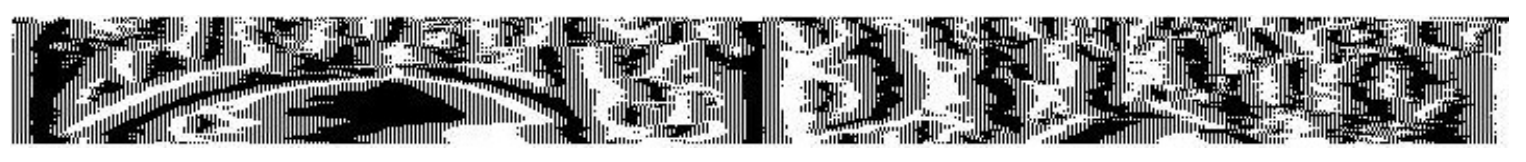

(b)

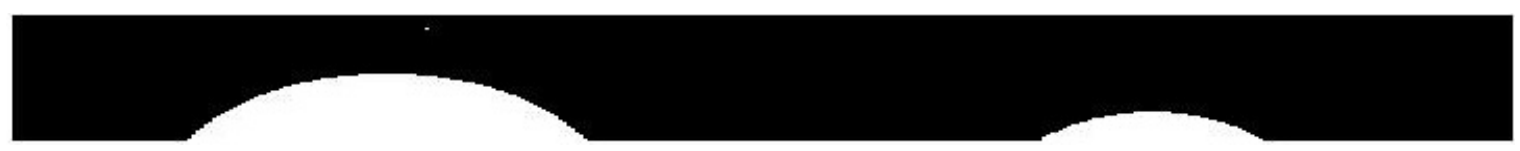

(c)

Figure 3.4: A trivial solution: Stitching halves of normalized images from the source and the target. (a) Iris pattern obtained by stitching. (b) Iris template of the stitched image. (c) Corresponding mask for iris template of the stitched image. 


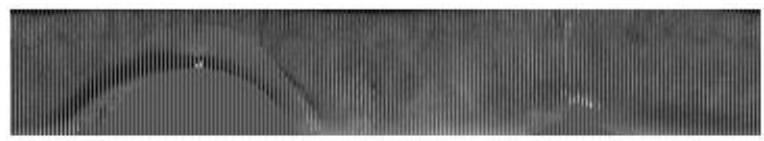

(a)

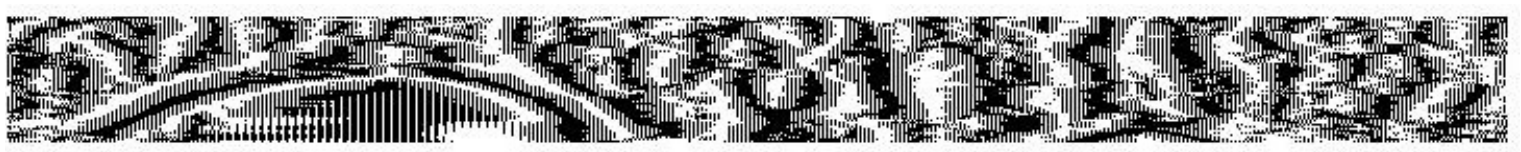

(b)

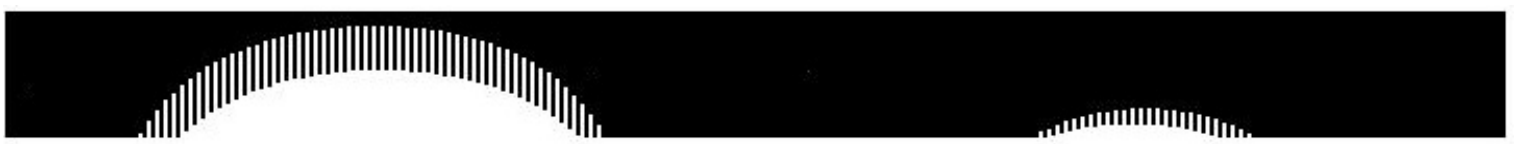

(c)

Figure 3.5: A trivial solution: Mixing normalized iris images uniformly. (a) Iris pattern obtained by uniformly mixing two normalized iris patterns. (b) Iris template of the uniformly mixed image. (c) Corresponding mask for iris template of the uniformly mixed image. 


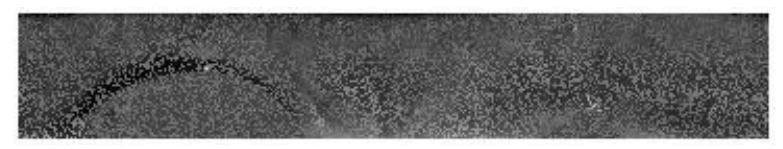

(a)

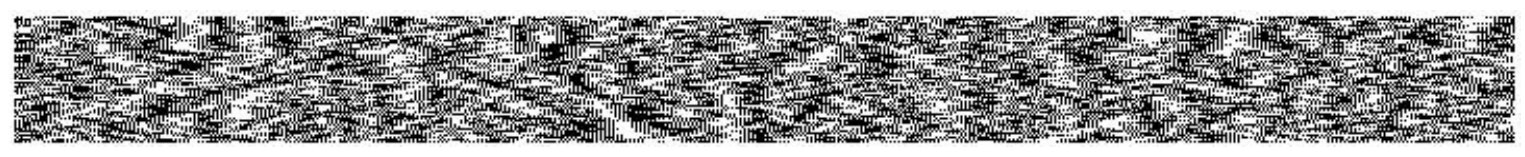

(b)

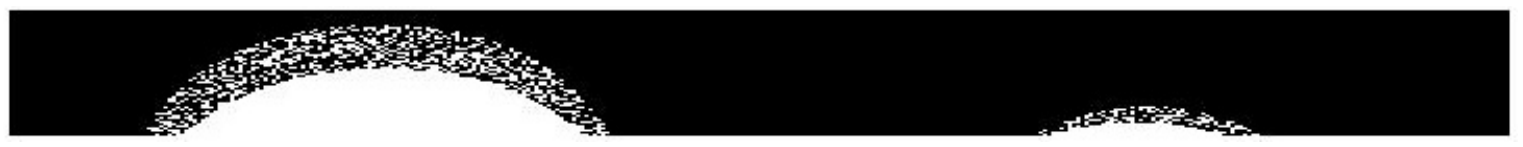

(c)

Figure 3.6: An alternative approach: Mixing normalized iris images randomly. (a) Iris pattern obtained by randomly mixing two normalized iris patterns. (b) Iris template of the randomly mixed image. (c) Corresponding mask for iris template of the randomly mixed image. 


\subsection{Proposed Scheme}

This study introduces a model which can be used to print a pattern onto a non-cosmetic transparent contact lens with the purpose of hosting two identities. In this regard, it is different from previous work on generating synthetic irides [23-26]. The goal of this model is to select certain pixels from the target iris pattern and copy those pixels onto the source iris pattern in order to create an optimum composite iris pattern that satisfies the following properties:

- The HD between the composite image and the target image is low enough that the composite image can be recognized as the target image by the recognition system.

- The HD between the composite image and the original source image is low enough that the composite image can be recognized as the source image by the recognition system.

- The process results in a non-periodic unique pattern so that the composite image can avoid potential detection by spoof detection algorithms.

- The percentage of copied pixels is as low as possible so that the copying process is more efficient than copying $50 \%$ of the target iris pattern.

We propose a scheme which guides the pixel selection process by examining and comparing the phase and intensity profiles of two pixels which are on the same location in the corresponding images. Assume that $P_{1}(x, y)$ is the pixel located at the Cartesian coordinates $(x, y)$ in the image $P_{1}$, and $P_{2}(x, y)$ is the pixel located at the Cartesian coordinates $(x, y)$ in the image $P_{2}$. Denote $I_{1}(x, y)$ as the intensity value of $P_{1}(x, y)$, and $I_{2}(x, y)$ as the intensity value $P_{2}(x, y)$. Denote $F_{1}(x, y)$ as the two bit phase information of $P_{1}(x, y)$, and $F_{2}(x, y)$ as the two bit phase information of $P_{2}(x, y)$. The proposed scheme copies $P_{2}(x, y)$ onto $P_{1}(x, y)$ when the following constraints hold:

- When $I_{1}(x, y) \leq I_{2}(x, y)$ and

- If $F_{2}(x, y)=00$ and $F_{1}(x, y)=10$

- If $F_{2}(x, y)=01$ and $F_{1}(x, y)=11$ and $x$ is even

- If $F_{2}(x, y)=10$ and $F_{1}(x, y)=11$ and $x$ is even 
- If $F_{2}(x, y)=11$ and $F_{1}(x, y)=10$

- Or when $I_{1}(x, y)>I_{2}(x, y)$ and

- If $F_{2}(x, y)=10$ and $F_{1}(x, y)=00$

- If $F_{2}(x, y)=11$ and $F_{1}(x, y)=01$ and $x$ is even

- If $F_{2}(x, y)=00$ and $F_{1}(x, y)=01$ and $x$ is even

- If $F_{2}(x, y)=01$ and $F_{1}(x, y)=00$

This scheme was formed after exploring the characteristics of all the possible combinations of phase values and found to fulfil all the requirements to form the optimum composite iris pattern. The additional constraint "if $x$ is even" is included to skip every second bit that satisfies the constraints $F_{1}(x, y)=01$ or $F_{1}(x, y)=11$, to limit the number of bits copied and to decrease the hamming distance between the composite image and $P_{1}(x, y)$. Demonstration of the proposed scheme on the images of subjects shown in Figures 3.2 and 3.3 is given in Figure 3.7. The details of the proposed model can be found in pseudo-code format in Algorithm 2.

The HD between the source iris pattern $P_{1}$ and the target iris pattern $P_{2}$ is 0.47 . The HD between the composite iris pattern and the original iris pattern $P_{1}$ is 0.29 . The HD between the composite iris pattern and the original iris pattern $P_{2}$ is 0.25 . According to these results, the composite image is recognized both as $P_{1}$ and $P_{2}$ by the iris recognition systems since the resultant hamming distances are significantly below the 0.32 threshold. Note that the percentage of pixels copied from $P_{2}$ is $19.8 \%$ of $P_{2}$ excluding the mask area. Also, see that the composite image is a unique blend of $P_{1}$ and $P_{2}$, which does not contain periodicity due to unique characteristics of the original iris patterns.

Imprinting the difference pixels between the composite image and $P_{1}$ onto a transparent contact lens will result in a new type of patterned contact lenses. However, a certain orientation should be maintained when the lenses are inserted in the eye, since the pattern in the lens surface should block specific parts of the genuine iris. Toric soft contact lenses which are used to correct astigmatism can be employed for that purpose. Toric lenses must be kept at a very specific angle in the eye hence they do not rotate once placed in the eye. The lenses are produced with a special technique that makes them slightly heavier at the bottom to prevent spinning. By printing a pattern onto a toric 
soft lens as described in the proposed model, the spoofer might be able to deceive iris recognition systems.

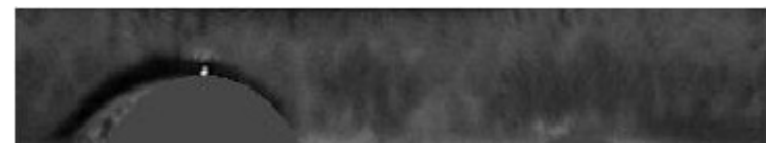

(a)

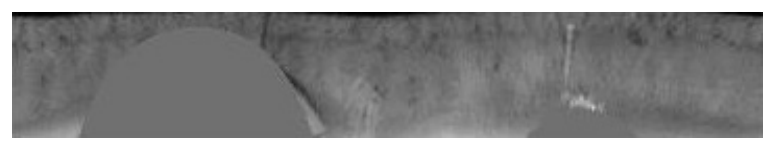

(b)

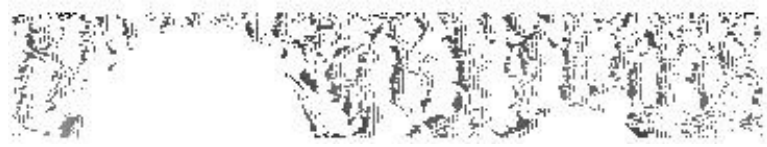

(c)

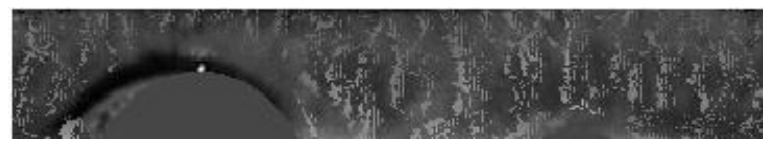

(d)

Figure 3.7: A demonstration of the proposed scheme. (a) Source iris pattern $P_{1}$. (b) Target iris pattern $P_{2}$. (c) Pixels to be copied. (d) The resultant composite iris pattern. 


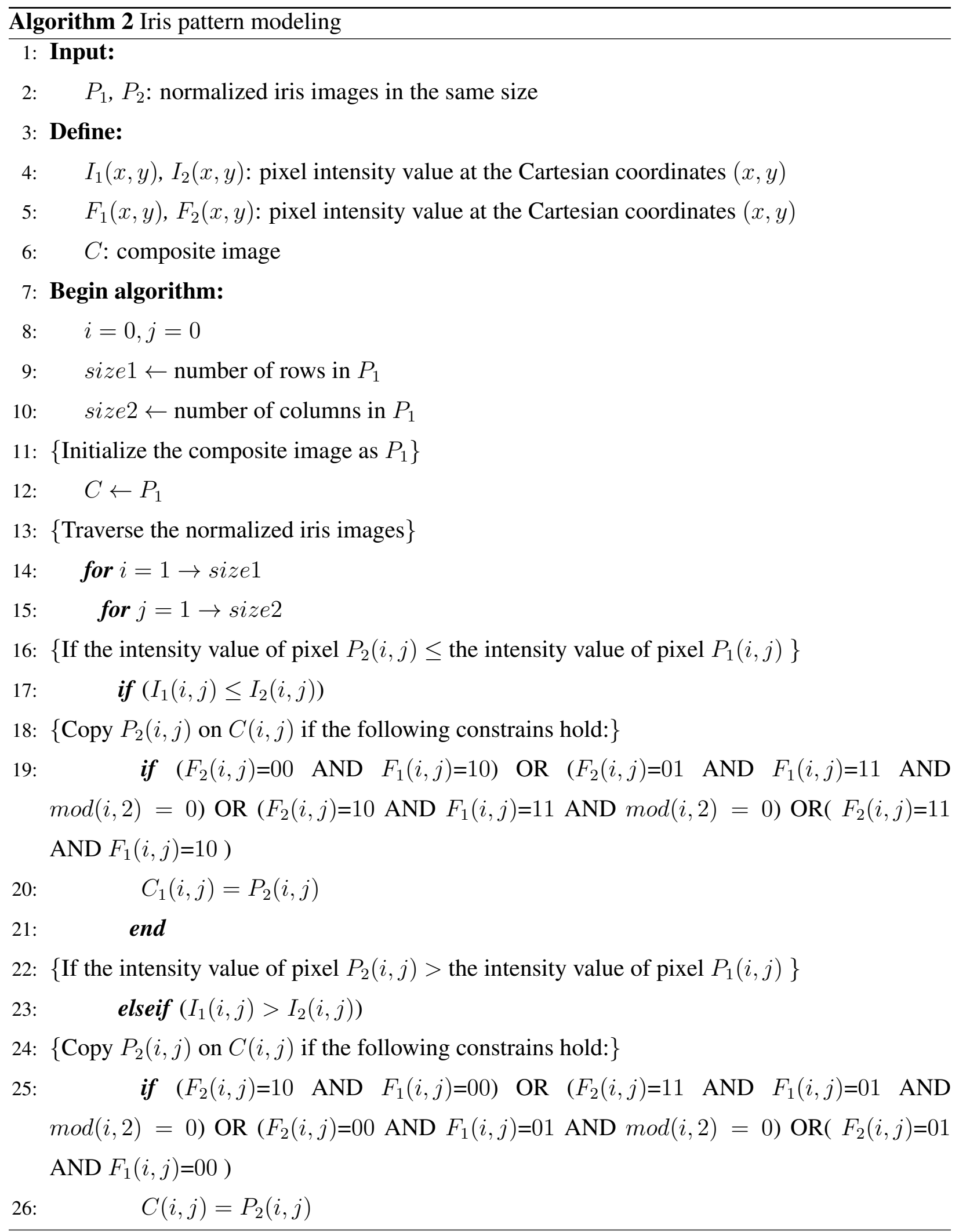




\begin{tabular}{lr}
\hline $27:$ & end \\
28: & end \\
29: & end \\
30: & end
\end{tabular}

31: End algorithm: return $C$

\subsection{Experimental Results}

The dataset used for this study was selected from ICE 2005. All the 98 subjects who were marked as without-lens subjects in the previous study were included in the experiment set with 3 images per subject. The total number of images were 294. By selecting a different pair of images each time, 9506 composite images were formed and the HD between the composite image and the source and target images were computed. Figures 3.8 - 3.16 show examples of target and source images along with the composite image formed by the proposed model. The HD relationships between different patterns are indicated with arrows in the figures.

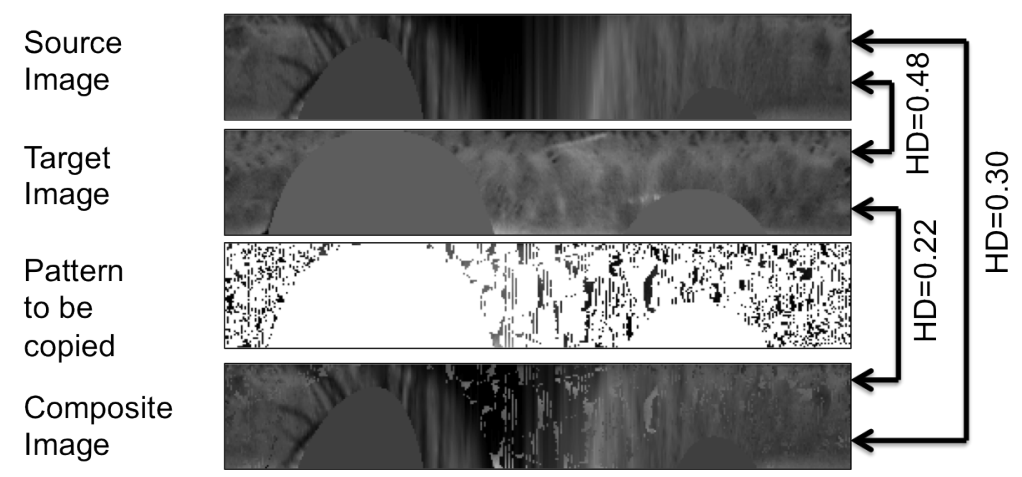

Figure 3.8: Example \#1: Source, target and composite iris patterns with the corresponding HDs.

Figure 3.18 shows the distribution of hamming distances between the composite image and the source image and the target image. The mean HD between the images of different identities is 0.48, whereas the mean HD between the composite iris and the source iris pattern is 0.28 and the mean HD between the composite iris and the target iris pattern is 0.29 . This indicates the efficacy of the proposed method.

Figure 3.19 shows the histogram of the percentage of pixels copied from the target to form 
Gizem Erdogan

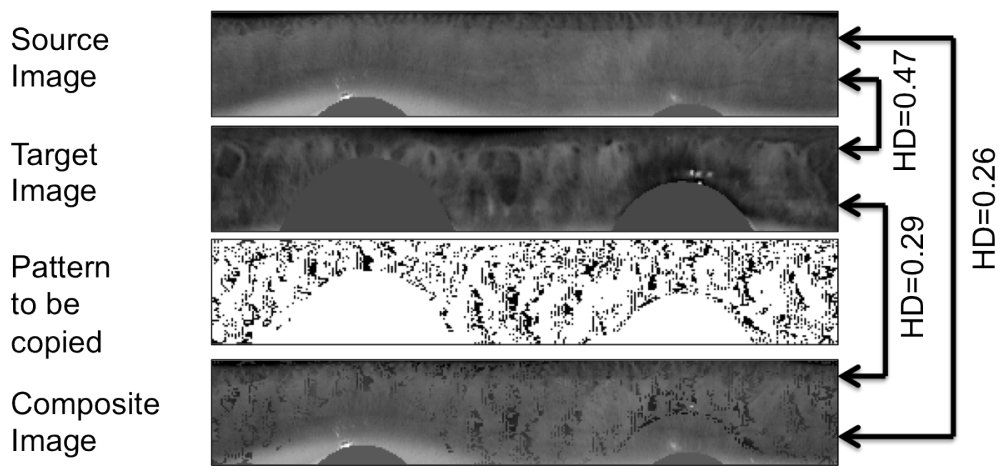

Figure 3.9: Example \#2: Source, target and composite iris patterns with the corresponding HDs.

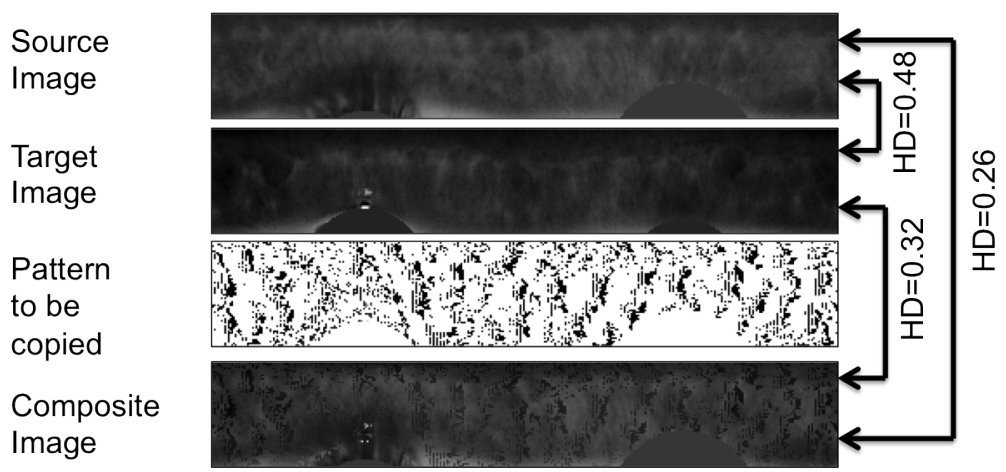

Figure 3.10: Example \#3: Source, target and composite iris patterns with the corresponding HDs.

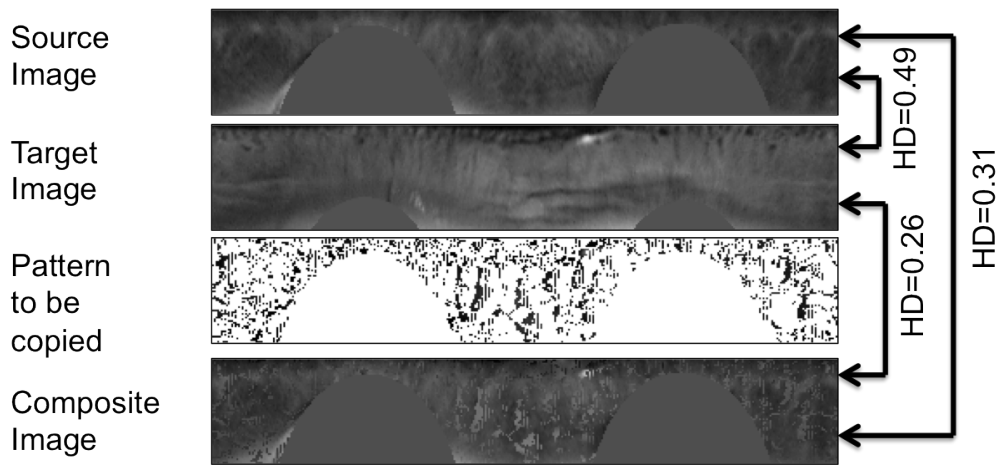

Figure 3.11: Example \#4: Source, target and composite iris patterns with the corresponding HDs.

each composite image. The mean of this distribution is $21.35 \%$, which implies that the proposed model is expected to host two identities on one iris pattern by copying around $21 \%$ of the target iris pattern onto the source iris pattern. 
Gizem Erdogan

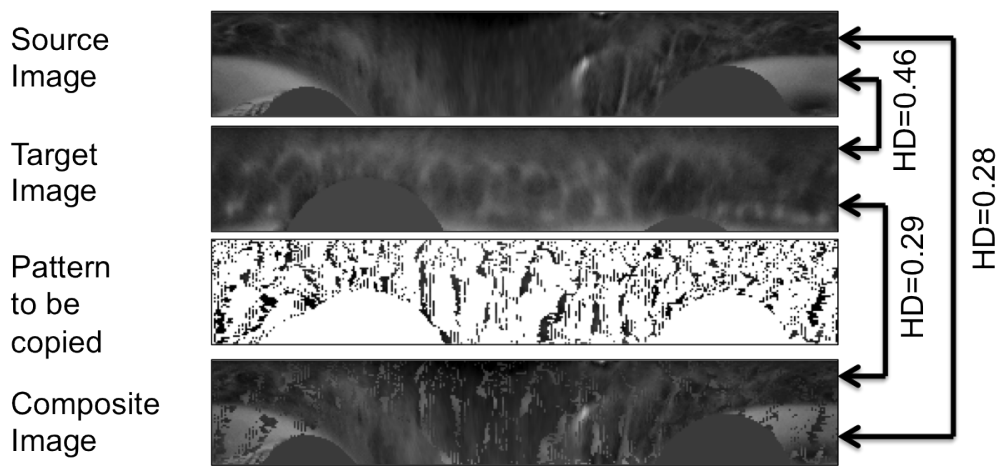

Figure 3.12: Example \#5: Source, target and composite iris patterns with the corresponding HDs.

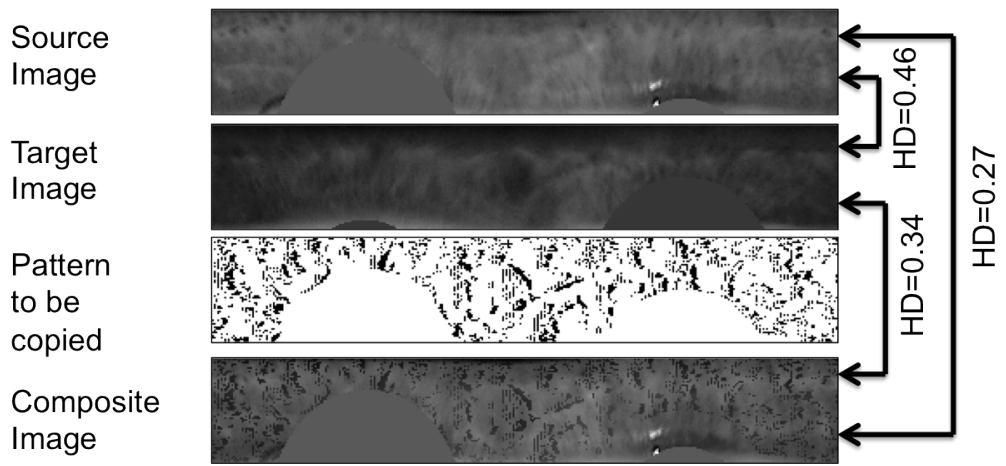

Figure 3.13: Example \#6: Source, target and composite iris patterns with the corresponding HDs.

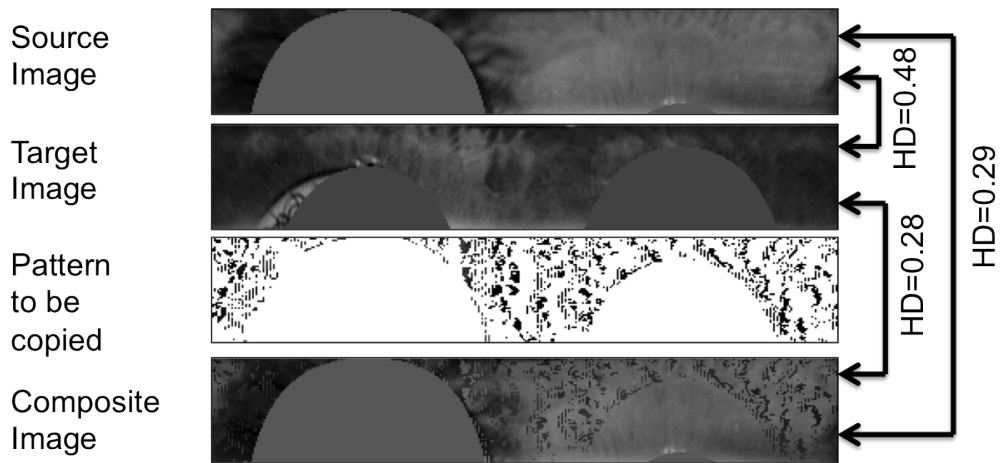

Figure 3.14: Example \#7: Source, target and composite iris patterns with the corresponding HDs.

Figure 3.20 shows the distribution of the genuine HDs across different images of each identity. There are 98 different identities with 3 images per identity in the dataset, hence 294 genuine scores are generated by matching different pairs of images. The histogram of genuine match scores is given in magenta, the mean genuine $\mathrm{HD}$ is 0.31 . 
Gizem Erdogan

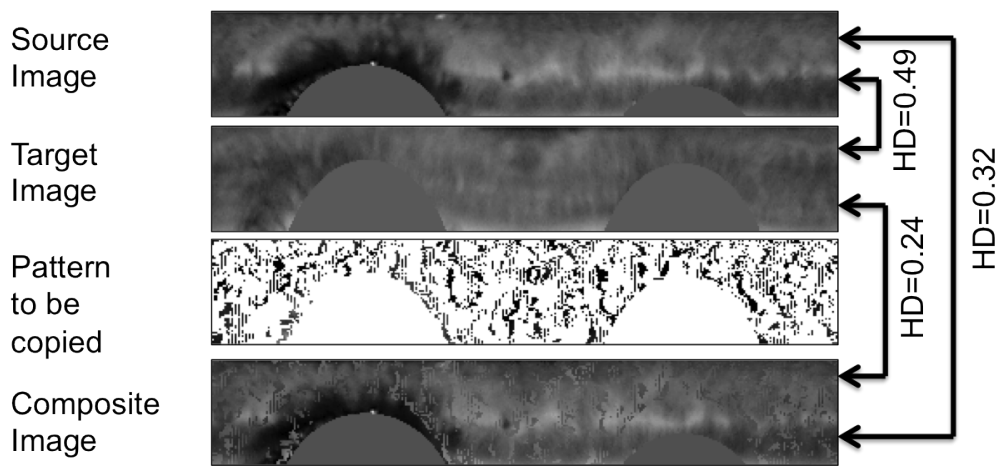

Figure 3.15: Example \#8: Source, target and composite iris patterns with the corresponding HDs.

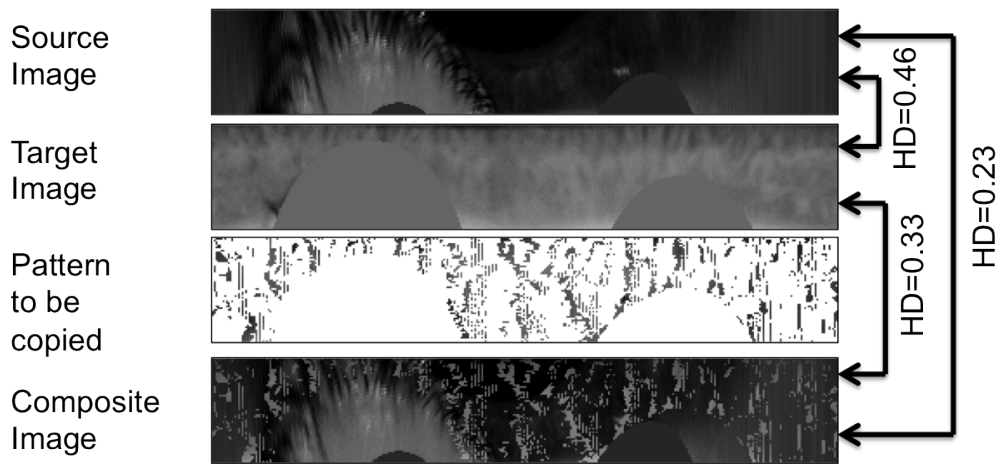

Figure 3.16: Example \#9: Source, target and composite iris patterns with the corresponding HDs.

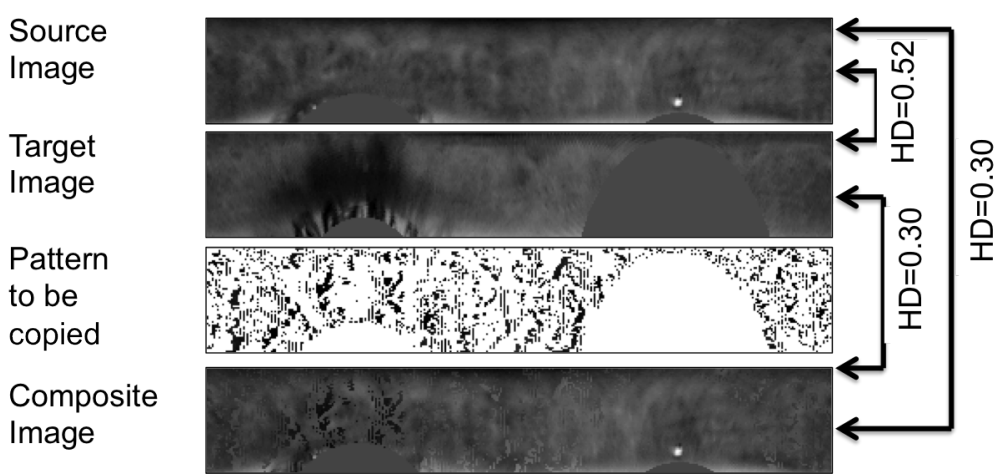

Figure 3.17: Example \#10: Source, target and composite iris patterns with the corresponding HDs.

Figure 3.21 brings in two other distributions. The HD between the composite iris pattern and the other samples from the source and target identities are calculated. The mean HD between the composite iris pattern and the samples of the source identity is 0.39 . The mean HD between the 


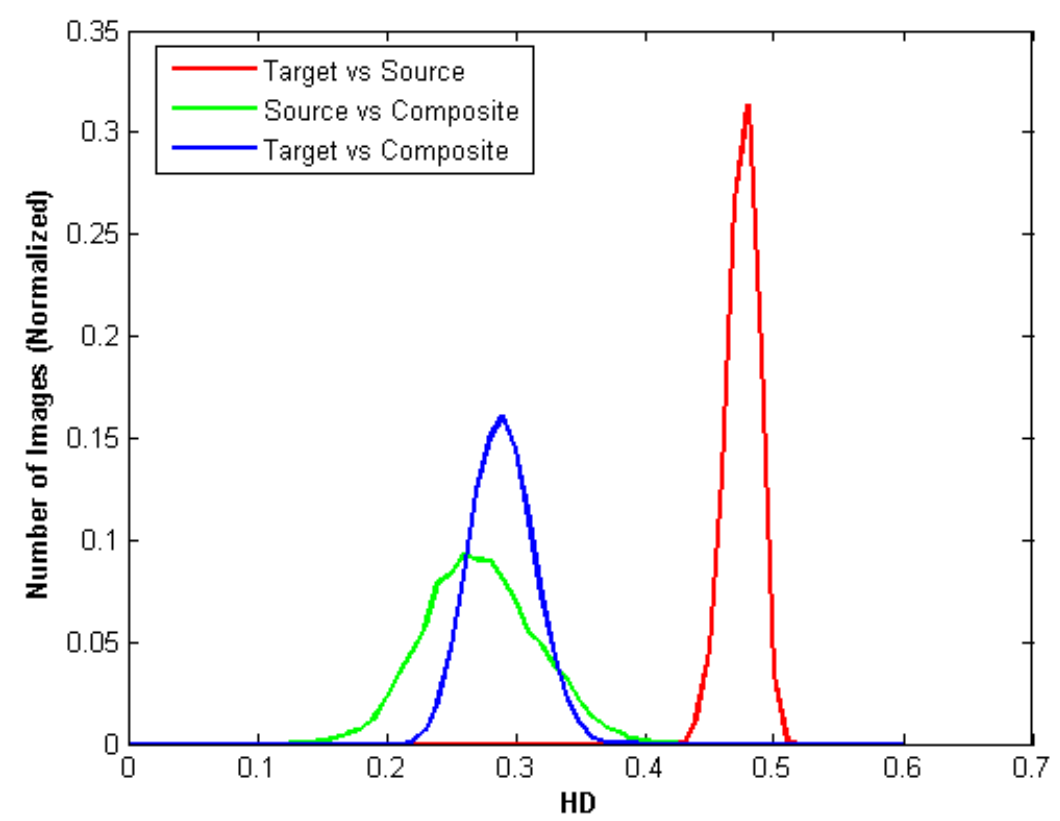

Figure 3.18: Distribution of HD between composite iris and the source and target iris patterns.

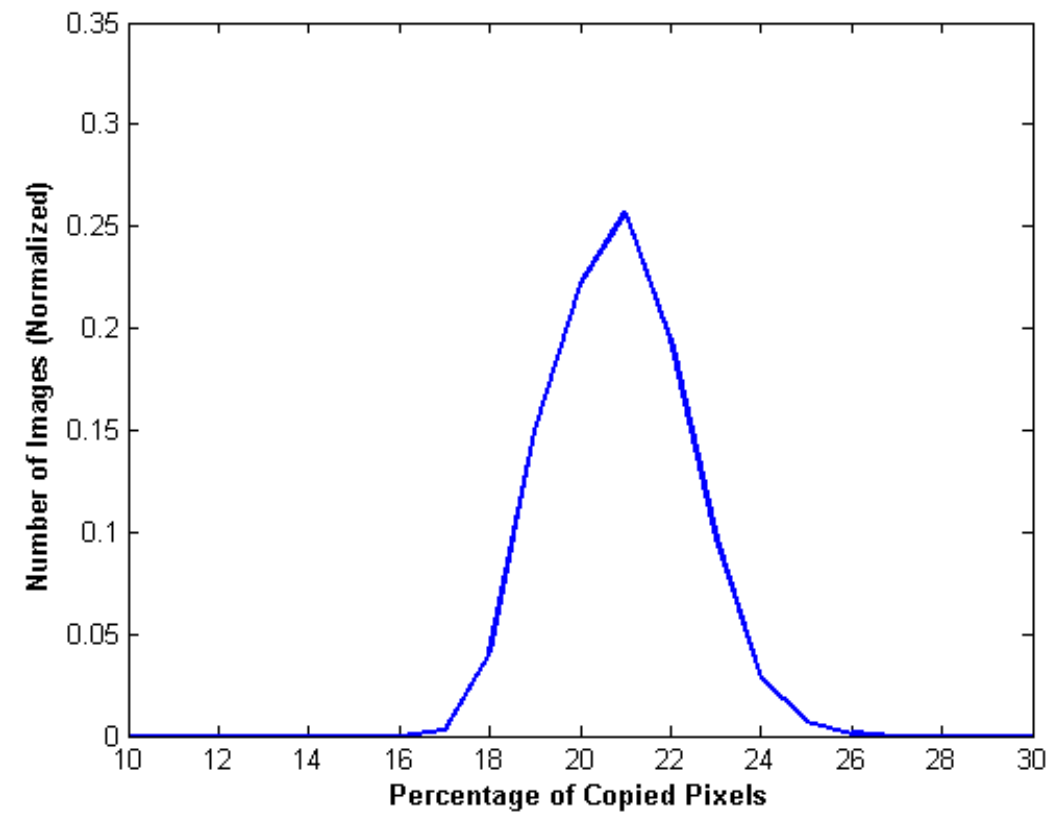

Figure 3.19: Histogram of the percentage of pixels copied onto the source image. 


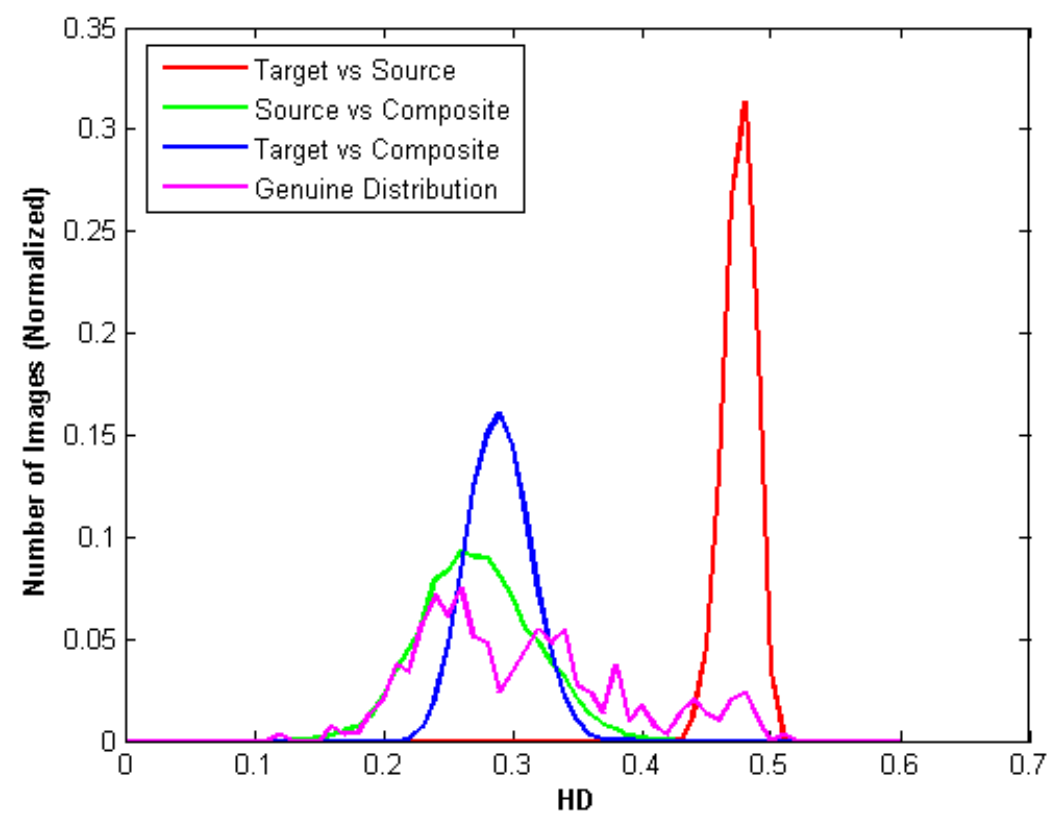

Figure 3.20: Distribution of HD between different patterns. Distribution of HD between genuine patterns are shown with magenta.

composite iris pattern and the samples of the target identity is 0.40 . This indicates the limitation of the current method. We are currently seeking ways to resolve this issue.

Figures 3.22 to 3.26 include examples of the most extreme cases of our experiments. Figure 3.22 shows the pair of source and target images which have the highest HD (0.51) in the dataset, and their related results. Figure 3.23 shows the case where the HD between the source image and the composite image is the highest, with 0.42 . In this case, the proposed method fails to host the source identity. However, it performs well for the target identity. Figure 3.24 shows the case where the HD between the target image and the composite image is the highest, with 0.39. In this case, the proposed method fails to host the target identity. On the other hand, it performs well for the source identity. Figure 3.25 shows the case where the sum of the HDs between the composite and the target image and the HD between the composite and the source image is the highest, with 0.68. Figure 3.26 shows the results when the percentage of copied pixels is the highest, with $26.8 \%$. 


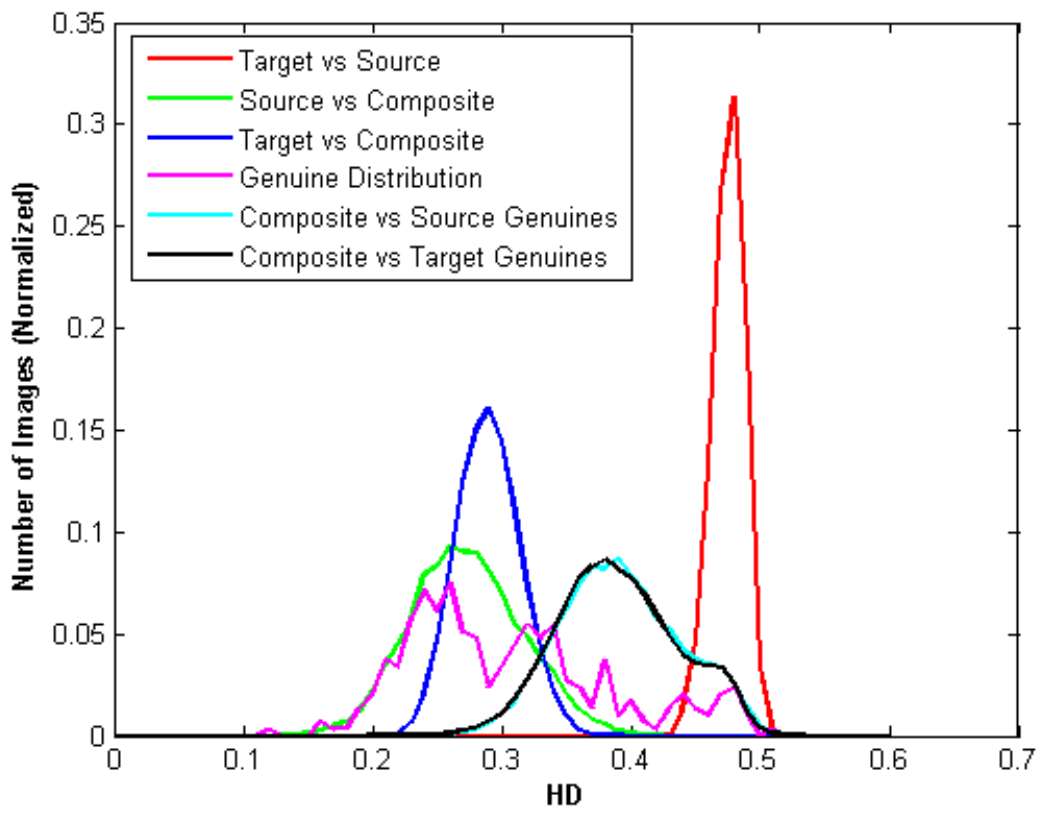

Figure 3.21: Distribution of HD between different patterns. Distribution of HD between the composite pattern and the other samples of its source identity are shown in cyan. Distribution of HD between the composite pattern and the other samples of its target identity are shown in black.

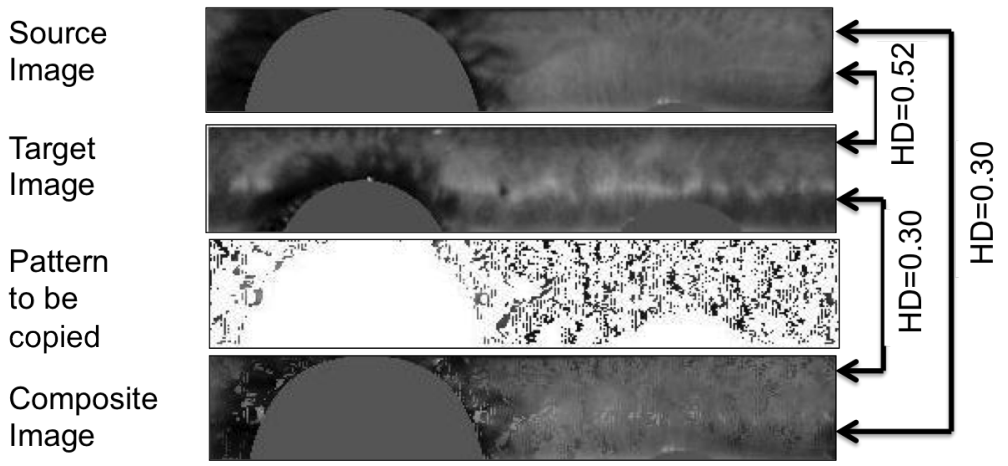

Figure 3.22: Example \#11: The pair of source and target images which have the highest HD. 


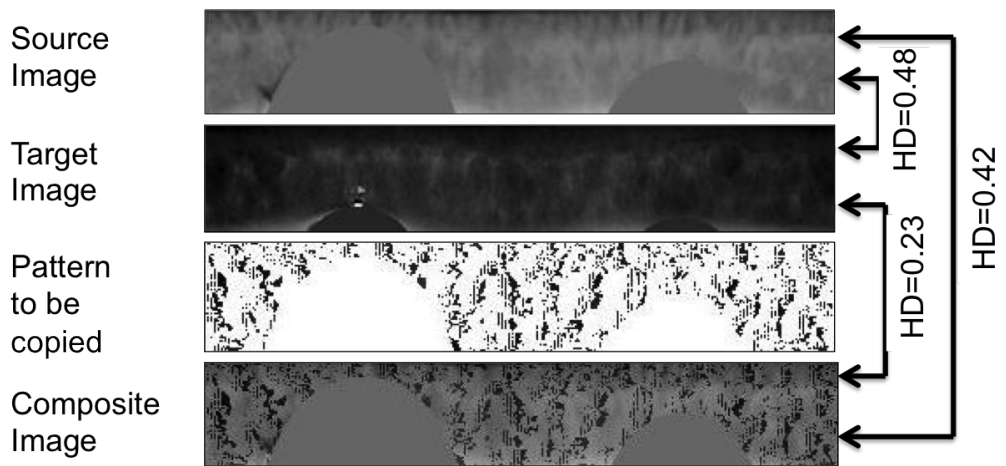

Figure 3.23: Example \#12: The case where the HD between the source image and the composite image is the highest.

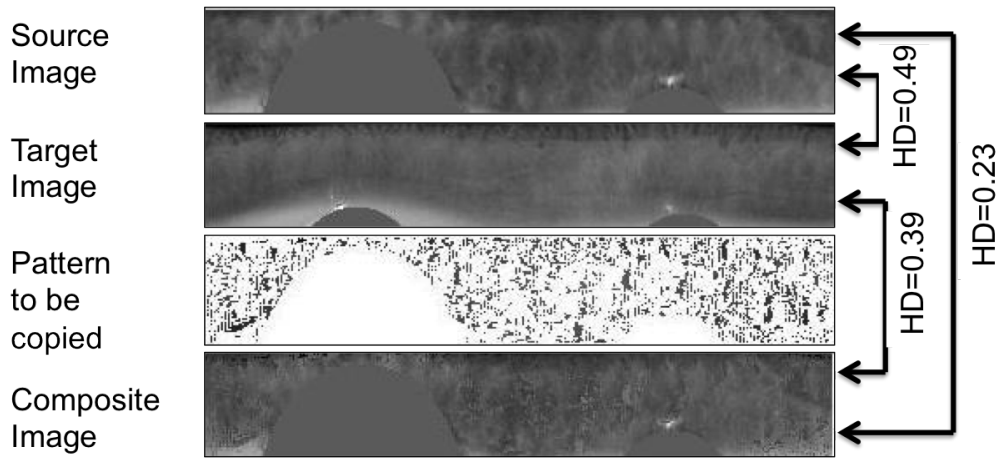

Figure 3.24: Example \#13: The case where the HD between the target image and the composite image is the highest.

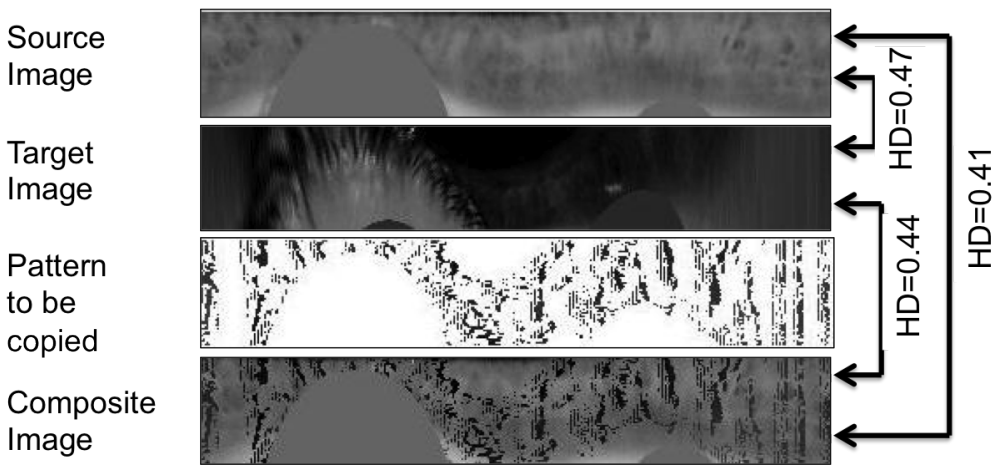

Figure 3.25: Example \#14: The case where the sum of the HDs between the composite and the target image and the HD between the composite and the source image is the highest. 


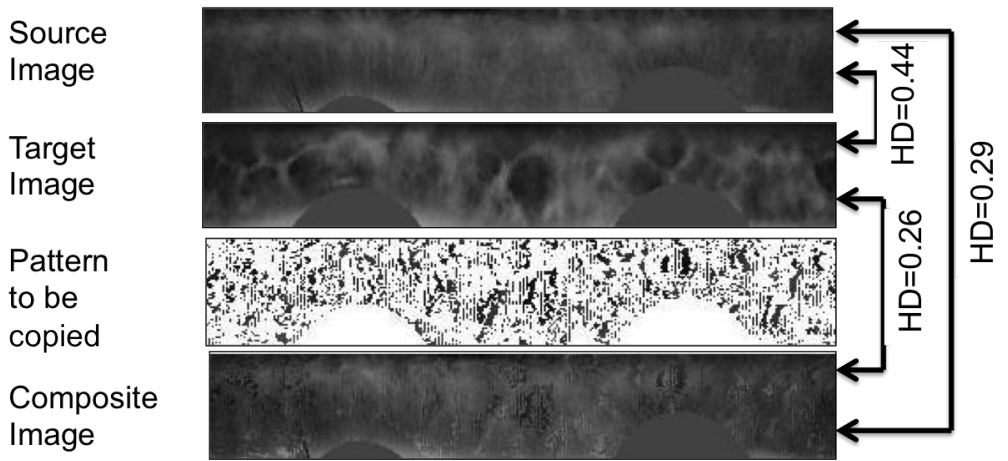

Figure 3.26: Example \#15: The case with the highest percentage of pixels copied, and the related results. 
Gizem Erdogan

\subsection{Feasibility of Spoof Attacks with the Proposed Model}

Experimental results provided in Section 3.3 highlight the significance of the proposed model and suggest that this model can potentially be used for creating patterned contact lenses. However, creating patterned contact lenses with the intention of facilitating dual identity, is not easily feasible. In other words, an adversary intending to exploit this approach for spoofing identities, has to overcome several challenges. Some of these challenges are listed below:

- The spoofer has to gain access to the iris pattern of the target identity to perform the described attack. Typically, iris databases do not keep explicit information regarding the identities of the subjects. Hence, the spoofer has to covertly capture iris images of the target identity in the NIR spectrum by their own means. Image acquisition is not achievable without the necessary gadgets and full cooperation of the target identity.

- The spoofer has to possess toric contact lenses to imprint the sparse pattern onto the lens. Unless the spoofer already has access to toric lenses (e.g., if he has astigmatism), he has to produce non-corrective transparent contact lenses with a mechanism that can adjust and secure the position of the lens in the eye.

- The spoofer has to possess expert level knowledge in iris biometrics. He must have access to iris recognition source codes and must be able to segment and normalize irides. He must also have the necessary skills to implement the proposed algorithm. Moreover, the spoofer must be able to re-wrap the normalized iris into a circular iris pattern.

- Imprinting patterned contact lenses with the proposed model has not been attempted before. Hence the DPI (dots per inch) value of the printer, which is needed to produce the desired output, is not known. However, this value might be higher than that used by traditional cosmetic contact lens printers.

Producing patterned contact lenses with the proposed model requires a well financed operation, and cannot be achieved by non-experts in the field. Thus, the probability of attacking iris recognition systems with the proposed model can be deemed to be minimal. 


\section{Chapter 4}

\section{Conclusion}

\subsection{Summary and Future Work}

In the first part of this thesis, the problem of automatically detecting non-cosmetic soft contact lenses in ocular images is investigated. This topic is challenging because non-cosmetic soft contact lenses are not easily discernible once they are placed in the eye. The proposed method operates under the principle that the boundary of a soft contact lens is at least partly visible against the sclera. This visible part of the boundary is detected by examining the intensity profiles of the pixels located in the vicinity of the outer limbus boundary of the iris. Our technique requires two tunable parameters ( $\alpha$ and $\tau$ ) which can be set according to the anticipated with-lens and withoutlens subject ratio in the datasets. However, in some real-world scenarios, an exact value of this ratio may not be easily anticipated.

Experiments were performed on two different databases. Approximately one third of the ICE 2005 database and the entire MBGC Iris database were utilized to evaluate the proposed method. The overall classification accuracy achieved in image level experiments was $76 \%$ in ICE 2005 and $66.8 \%$ in MBGC Iris, with correct detection rates of $72 \%$ and $70 \%$, respectively. To eliminate the subjectivity due to manual labeling of data, subject level experiments were performed also. The overall accuracy obtained in subject level experiments was $76 \%$ in ICE 2005 and $76.3 \%$ in MBGC Iris, with correct detection rates of $74 \%$ and $70 \%$, respectively. The algorithm failed in the case of images with inconsistent illumination and defocus blur. Our target for the future work is to overcome these two challenges. To our knowledge there is only one related work in the literature 
by Kywe et al. that can detect non-cosmetic soft contact lenses but their technique requires additional hardware and is highly dependent on the temperature and humidity of the experimental environment [20]. The results of both studies suggest that this topic is open to further research.

In the second part of this thesis, a novel model for creating a dual identity iris image, which can potentially be employed to create a novel type of patterned contact lenses, is proposed. The proposed model introduces a scheme for copying pixels from a target iris pattern onto a source pattern to form a composite image, while keeping the HD between the composite image and the target and source images within acceptable limits. Modeling patterned contact lenses with this scheme might enable a spoofer to be recognized as the target identity, as well as the source identity. Additionally, this method requires fewer number of pixels to be copied $(\sim 21 \%)$, and is more efficient than imprinting regular patterned contact lenses. The proposed method can be implemented by acquiring a target individual's iris pattern and copying certain pixels onto a toric contact lens according to the proposed scheme. Since the pattern to be copied is unique and sparse, the pattern on the clear lens is expected to blend well with the genuine iris pattern. This might make it difficult for texture-based spoof detection mechanisms to detect the spoofing process. However, the non-cosmetic contact lens detection method proposed in Chapter 2 is not expected to show any degradation of performance, since that method focuses on detecting the outer boundary of the transparent lens. Misuse of this work in a real-world spoofing scenario is also explained to be very challenging for various reasons. Although this study does not cover the possibility of producing patterned lenses, we believe that the modeling aspect of this study is significant and contributes to the ongoing research in iris spoofing. 


\section{References}

[1] Jason Nichols, "Annual report: Contact lenses 2011," Contact Lens Spectrum, vol. 27, pp. 20 -25, January 2012. [Online]. Available: http://www.clspectrum.com/articleviewer.aspx? articleID $=106550$

[2] "Contact lenses," Retrieved in June 2011, from http://www.contactlenscouncil.com/ pcon-stats.htm/.

[3] “Iris,” Retrieved in Nov. 2012, from http://en.wikipedia.org/wiki/Iris_(anatomy).

[4] A. Ross, "Iris recognition: The path forward," Computer, vol. 43, no. 2, pp. 30-35, Feb. 2010.

[5] J. Daugman and C. Downing, "Epigenetic randomness, complexity and singularity of human iris patterns," Proceedings of the Royal Society of London. Series B: Biological Sciences, vol. 268, no. 1477, pp. 1737-1740, 2001.

[6] J. Daugman, "How iris recognition works," IEEE Transactions on Circuits and Systems for Video Technology, vol. 14, no. 1, pp. 21-30, 2004.

[7] “Contact lens,” Retrieved in October 2012, from http://en.wikipedia.org/wiki/Contact_lens.

[8] “Types of contact lenses," Retrieved in Dec. 2011, from http://www.fda.gov/medicaldevices/ productsandmedicalprocedures/homehealthandconsumer/consumerproducts/contactlenses/ ucm062319.htm/.

[9] Gary Heiting, "Silicone hydrogel contact lenses," Retrieved in October 2012, from http:// www.allaboutvision.com/contacts/silicone-hydrogel.htm.

[10] X. He, S. An, and P. Shi, "Statistical texture analysis-based approach for fake iris detection using support vector machines," in Advances in Biometrics, ser. Lecture Notes in Computer Science, S.-W. Lee and S. Li, Eds. Springer Berlin Heidelberg, 2007, vol. 4642, pp. 540-546. [Online]. Available: http://dx.doi.org/10.1007/978-3-540-74549-5_57

[11] Z. He, Z. Sun, T. Tan, and Z. Wei, "Efficient iris spoof detection via boosted local binary patterns," in Proceedings of the Third International Conference on Advances in Biometrics. Berlin, Heidelberg: Springer-Verlag, 2009, pp. 1080-1090. [Online]. Available: http://dx.doi.org/10.1007/978-3-642-01793-3_109 
[12] E. C. Lee, K. R. Park, and J. Kim, "Fake iris detection by using purkinje image," in Proceedings of the International Conference on Advances in Biometrics. Berlin, Heidelberg: Springer-Verlag, 2006, pp. 397-403. [Online]. Available: http: //dx.doi.org/10.1007/11608288_53

[13] Z. Wei, X. Qiu, Z. Sun, and T. Tan, "Counterfeit iris detection based on texture analysis," in Proceedings of the International Conference on Pattern Recognition (ICPR), 2008, pp. 1-4.

[14] J. Daugman, "Demodulation by complex-valued wavelets for stochastic pattern recognition," International Journal of Wavelets, Multiresolution and Information Processing, vol. 01, no. 01, pp. 1-17, 2003. [Online]. Available: http://www.worldscientific.com/doi/abs/10. $1142 / \mathrm{S} 0219691303000025$

[15] S. E. Baker, A. Hentz, K. W. Bowyer, and P. J. Flynn, "Degradation of iris recognition performance due to non-cosmetic prescription contact lenses," Computer Vision and Image Understanding, vol. 114, no. 9, pp. 1030 - 1044, 2010. [Online]. Available: http://www.sciencedirect.com/science/article/pii/S1077314210001360

[16] "Allergies of the eye," Retrieved in Nov. 2012, from http://www.kellogg.umich.edu/ patientcare/conditions/allergies.html.

[17] "Replacement schedule is a powerful driver for compliance," Retrieved in November 2012, from https://www.myalcon.com/education/technical-education/promoting-compliance. shtml.

[18] "Find the right contact lenses for you," Retrieved in June 2011, from http://www.webmd. com/eye-health/contact-lenses-colored-soft-hard-toric-bifocal/.

[19] H. Zhang, Z. Sun, and T. Tan, "Contact lens detection based on weighted LBP," in Proceedings of the International Conference on Pattern Recognition (ICPR), 2010, pp. 4279 -4282.

[20] W. Kywe, M. Yoshida, and K. Murakami, "Contact lens extraction by using thermo-vision," in Proceedings of International Conference on Pattern Recognition (ICPR), vol. 4, 2006, pp. 570-573.

[21] L. Masek and P. Kovesi, "Matlab source code for a biometric identification system based on iris patterns," http://www.csse.uwa.edu.au/ pk/studentprojects/libor/sourcecode.html, 2003.

[22] W. Barrett, "Detecting a contact lens during iris recognition," Retrieved in Oct. 2012, from http://www.engr.sjsu.edu/wbarrett/publications/Composite.pdf.

[23] A. Lefohn, B. Budge, P. Shirley, R. Caruso, and E. Reinhard, "An ocularist's approach to human iris synthesis," Computer Graphics and Applications, vol. 23, no. 6, pp. 70-75, Nov.Dec. 2003.

[24] J. Cui, Y. Wang, J. Huang, T. Tan, and Z. Sun, "An iris image synthesis method based on PCA and super-resolution," in Proceedings of the 17th International Conference on Pattern Recognition, 2004, vol. 4, Aug. 2004, pp. $471-474$. 
[25] S. Makthal and A. Ross, "Synthesis of iris images using markov random fields," Proceedings of the European Signal Processing Conference (EUSIPCO), 2005.

[26] S. Shah and A. Ross, "Generating synthetic irises by feature agglomeration," in IEEE International Conference on Image Processing, Oct. 2006, pp. 317-320. 